\title{
Spectral optical monitoring of 3C 390.3 in 1995-2007
}

\section{Light curves and flux variation in the continuum and broad lines ${ }^{\star}$}

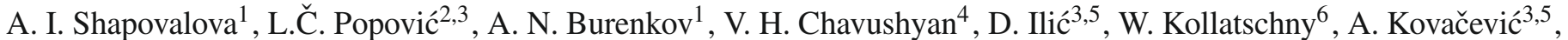 \\ N. G. Bochkarev ${ }^{7}$, L. Carrasco ${ }^{4}$, J. León-Tavares ${ }^{8}$, A. Mercado ${ }^{9}$, J. R. Valdes ${ }^{4}$, V. V. Vlasuyk ${ }^{1}$, and E. de la Fuente ${ }^{10}$ \\ 1 Special Astrophysical Observatory of the Russian AS, Nizhnij Arkhyz, Karachaevo-Cherkesia 369167, Russia \\ e-mail: ashap@sao.ru \\ 2 Astronomical Observatory, Volgina 7, 11160 Belgrade 74, Serbia \\ 3 Isaac Newton Institute of Chile, Yugoslavia Branch \\ 4 Instituto Nacional de Astrofísica, Óptica y Electrónica, Apartado Postal 51, CP 72000, Puebla, Pue. México \\ 5 Department of Astronomy, Faculty of Mathematics, University of Belgrade, Studentski trg 16, 11000 Belgrade, Serbia \\ 6 Institut für Astrophysik, Friedrich-Hund-Platz 1, Göttingen, Germany \\ 7 Sternberg Astronomical Institute, Moscow, Russia \\ 8 Aalto University Metsähovi Radio Observatory, Metsähovintie 114, 02540 Kylmälä, Finland \\ 9 Universidad Politécnica de Baja California, Mexicali B.C., México \\ 10 Instituto de Astronomia y Meteorologia, Dpto. de Física CUCEI, Universidad de Guadalajara, Av. Vallarta 2602, CP 44130, \\ Guadalajara, Jalisco, México
}

Received 22 January 2010 / Accepted 8 March 2010

\section{ABSTRACT}

\begin{abstract}
Context. We present the results of the long-term (1995-2007) spectral monitoring of the broad-line radio galaxy 3C 390.3, a well known AGN with double-peaked broad emission lines, usually assumed to be emitted from an accretion disk.

Aims. To explore dimensions and structure of the BLR, we analyze the light curves of the broad $\mathrm{H} \alpha$ and $\mathrm{H} \beta$ line fluxes and the continuum flux. To detect variations in the BLR, we analyze the $\mathrm{H} \alpha$ and $\mathrm{H} \beta$ line profiles, as well as the change in the line profiles during the monitoring period.

Methods. We attempt first to find a periodicity in the continuum and $\mathrm{H} \beta$ light curves, finding that there is a high probability of measuring quasi-periodical oscillations. Using the line shapes and their characteristics (such as e.g., peak separation and their intensity ratio, or $F W H M$ ) of broad $\mathrm{H} \beta$ and $\mathrm{H} \alpha$ lines, we discuss the structure of the BLR. We also cross-correlate the continuum flux with $\mathrm{H} \beta$ and $\mathrm{H} \alpha$ lines to determine the dimensions of the BLR.

Results. During the monitoring period, we found that the broad emission component of the $\mathrm{H} \alpha$ and $\mathrm{H} \beta$ lines, and the continuum flux varied by a factor of $\approx 4-5$. We also detected different structure in the line profiles of $\mathrm{H} \alpha$ and $\mathrm{H} \beta$. An additional central component appears to be present and superimposed on the disk emission. In the period of high activity (after 2002), $\mathrm{H} \beta$ became broader than $\mathrm{H} \alpha$ and red wing of $\mathrm{H} \beta$ was higher than that of $\mathrm{H} \alpha$. We detected time lags of $\sim 95$ days between the continuum and $\mathrm{H} \beta$ flux, and about 120 days between the continuum and $\mathrm{H} \alpha$ flux.

Conclusions. Variations in the line profiles, as well as correlation between the line and continuum flux during the monitoring period, are consistent with a disk origin of the broad lines and the possible contribution of some additional region and/or some kind of perturbation in the disk.
\end{abstract}

Key words. galaxies: active - quasar: individual: 3C 390.3 - line: profiles

\section{Introduction}

Active galactic nuclei (AGN) often exhibit variability in the broad emission lines. The region where broad lines are formed (hereinafter BLR - broad line region) is close to the central supermassive black hole and may hold basic information about the formation and fueling of AGN.

A long-term spectral monitoring of the nucleus of some AGN has revealed a time lag in the response of the broad emission lines relative to flux changes in the continuum. This lag depends on the size, geometry, and physical conditions of the BLR. Thus, the search for correlations between the nuclear continuum changes and flux variations in the broad emission lines

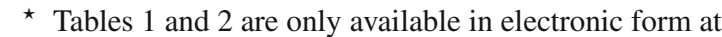
http://www . aanda.org may serve as a tool for mapping the geometrical and dynamical structure of the BLR (see e.g., Peterson 1993, and reference therein).

During the past decade, the study of the BLR in some objects has achieved considerable success, mainly because of the increasing number of coordinated multiwavelength monitoring campaigns through the international "AGN Watch" program (see e.g., Peterson 1999). Most of the objects, included in the AGN Watch, are radio-quiet Sy 1 galaxies and only one, 3C $390.3(z=$ 0.0561 ), is a well known broad-line radio galaxy. It is a powerful double-lobed FRII radio-galaxy with a relatively strong compact core. The two extended lobes, at a position angle of $144^{\circ}$, each one with a hot spot, are separated by about $223^{\prime \prime}$ (Leahy \& Perley 1991). A faint well-collimated thin jet at PA = $37^{\circ}$, connecting the core to the northern lobe, was observed by 
Leahy \& Perley (1995). The VLBI observations at $5 \mathrm{GHz}$ show evidence of the superluminal motion (with $v / c \sim 4$ ) in this parsec-scale jet (Alef et al. 1988, 1996).

In a similar way to about $10 \%$ of radio-loud AGN, 3C 390.3 emits very broad, double-peaked emission lines. A number of possible models have been suggested to explain double-peaked line profiles: supermassive binary black holes (Gaskell 1996), outflowing biconical gas streams (Zheng 1996), or emission from an accretion disk (Perez et al. 1988; Rokaki et al. 1992). The strong variability of this object, in both continuum and emission lines is well known (Barr et al. 1980, 1983; Yee \& Oke 1981; Netzer 1982; Clavel \& Wamsteker 1987; Veilleux \& Zheng 1991; Wamsteker et al. 1997; O'Brien et al. 1998; Dietrich et al. 1998; Shapovalova et al. 2001; Sergeev et al. 2002; Tao et al. 2008; Gupta et al. 2009). The object is also a highly variable X-ray source, which has a spectrum exhibiting a broad Fe K $\alpha$ line (Inda et al. 1994; Eracleous et al. 1996; Wozniak et al. 1998). During a multiwavelength monitoring campaign in 1995, several, large-amplitude, X-ray flares were observed in 3C 390.3; in one of them, the X-ray flux increased by a factor of 3 in a period of 12 days (Leighly et al. 1997). The X-ray flux also varies on scales of several days (Gliozzi et al. 2003; Gupta et al. 2009), exhibiting greater variation for a smaller energy range (Gliozzi et al. 2003).

In an analysis of IUE spectra of 3C 390.3 obtained during 1978-1986, Clavel \& Wamsteker (1987) detected a variability time lag of 50 and 60 days for the broad C IV $\lambda 1549$ and $\operatorname{Ly} \alpha$ emission lines, relative to the UV-continuum changes. However, from a reanalysis of the same spectral data, Wamsteker et al. (1997) derived a lag of $116 \pm 60$ days for C IV and $143 \pm$ 60 days for $\operatorname{Ly} \alpha$. Furthermore, from the IUE monitoring data from December 1994 to March 1996, O’Brien et al. (1998) measured a corresponding lag of 35 days for the C IV $\lambda 1549$ emission line and 60 days for $\operatorname{Ly} \alpha$. From the optical monitoring of 3C 390.3 during 1994-1995, Dietrich et al. (1998) derived, however, a time lag of about 20 days for the Balmer lines, i.e., their response with respect to changes in the X-ray continuum. Furthermore, a temporal lag between the optical and the UV or $\mathrm{X}$-ray continua was not detected. From the spectrophotometric monitoring of 3C 390.3 in 1995-1999, Shapovalova et al. (2001) (Paper I, hereinafter) found that the lag of the $\mathrm{H} \beta$ emission line relative to the continuum flux changes was $\sim 100$ days. Sergeev et al. (2002) inferred a lag between the continuum and $\mathrm{H} \beta$ variations for the centroid CCF of $\sim 89$ days. The UV-bump, usually observed in a large number of Seyfert galaxies, is very weak or even absent in 3C 390.3 (Wamsteker et al. 1997).

Studies of the variations in both the continuum and broad emission-line profiles and their correlations can provide information about the BLR physics (see e.g., Shapovalova et al. 2009). The double-peaked broad lines of 3C 390.3 are indicative of accretion disk emission, therefore long period spectral variation in 3C 390.3 can help us determine the nature of accretion disks. In this paper, we present the results of the spectral ( $\mathrm{H} \alpha$ and $\mathrm{H} \beta$ ) monitoring of 3C 390.3 during the period between 1995 and 2007. This work proceeds the long-term monitoring program for 3C 390.3 and our first results were published in 2001 (Paper I). In this paper, we present spectral data between 1995 and 2007, and we analyze the continuum and $\mathrm{H} \alpha, \beta$ line variations. The paper is organized as follow: in Sect. 2 we describe our observations and data reduction, in Sect. 3 we present the analysis of the observations, in Sect. 4 we discuss our results, and in Sect. 5 we outline our conclusions.

\section{Observations and data reduction}

\subsection{Spectral observations}

Spectra of 3C 390.3 (during 158 nights) were taken with the $6 \mathrm{~m}$ and $1 \mathrm{~m}$ telescopes of the SAO RAS (Russia, 1995-2007) and with INAOE's $2.1 \mathrm{~m}$ telescope of the "Guillermo Haro Observatory" (GHO) at Cananea, Sonora, México (1998-2007). They were acquired using long-slit spectrographs, equipped with CCD detector arrays. The typical wavelength interval covered was from $4000 \AA$ to $7500 \AA$, the spectral resolution varied between 5 and $15 \AA$, and the $S / N$ ratio was $>50$ in the continuum near $\mathrm{H} \alpha$ and $\mathrm{H} \beta$. Spectrophotometric standard stars were observed every night. The log of spectroscopic observations is given in Table 1 (available electronically only).

The spectrophotometric data reduction was carried out using either the software developed at SAO RAS or the IRAF package for the spectra obtained in México. The image reduction process included bias and flat-field corrections, cosmic ray removal, 2D wavelength linearization, sky spectrum subtraction, addition of the spectra for every night, and relative flux calibration based on standard star observations.

\subsection{Absolute calibration (scaling) of the spectra}

The standard technique of the flux calibration of spectra (i.e., comparison with stars of known spectral energy distribution) is not precise enough for the study of AGN variability, since even under good photometric conditions, the accuracy of spectrophotometry is not superior to $10 \%$. Therefore we used standard stars only for a relative calibration.

For the absolute calibration, the fluxes of the narrow emission lines are adopted for scaling the AGN spectra, because they are known to remain constant on timescales of tens of years (Peterson 1993).

However, for 3C 390.3 there has been discussion about the variability of the narrow lines. We considered this in more detail in Paper I and found that in the case of 3C 390.3 there is no reliable evidence of the [O III] $\lambda \lambda 4959+5007$ flux variability in timescales of between months and years. Therefore, spectra were scaled by the [O III] $\lambda \lambda 4959+5007$ integrated line flux taken to be $1.7 \times 10^{-13} \mathrm{erg} \mathrm{s}^{-1} \mathrm{~cm}^{-2}$ (Veilleux \& Zheng 1991). To confirm this, we compared photometric fluxes in the $V$ filter with the spectral fluxes (close to observed $\lambda=5384 \AA$ ) obtained from spectra scaled by [OIII] $\lambda \lambda 4959,5007$. Spectra and photometric data were obtained using the aperture $2^{\prime \prime} \times 6^{\prime \prime}$, and $15^{\prime \prime}$, respectively. Both observations were performed in the same, or in nearby nights (separated by $<3$ days). The $V$ magnitudes were transformed into fluxes $F(V)$ using the equation (Alen 1973; Wamsteker 1981; Dietrich et al. 1998)

$\log F(V, 5500)=-0.4 V-8.439$.

Figure 1 shows a correlation between photometric and spectral fluxes in the monitoring period. The regression line corresponds to the equation:

$F(V)=(0.59 \pm 0.07)+(1.25 \pm 0.03) F(s p)$.

As can be seen from Fig. 1, there is a strong linear relationship between $F(V)$ and $F(s p)$ with the correlation coefficient $R=$ 0.99 . This indicates that the $[\mathrm{OIII}]$ lines do not vary (at least on a level of a few percent) on timescales of $\sim 13$ years, i.e. during our monitoring period.

The scaling of the blue spectra was performed using the method of Van Groningen \& Wanders (1992) modified by 


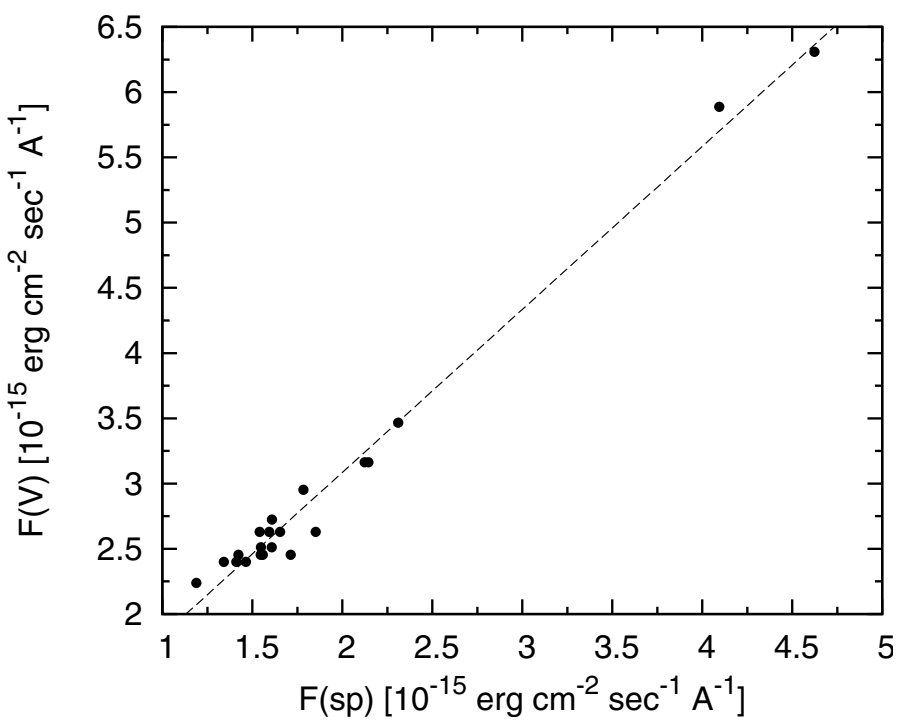

Fig. 1. The correlation between the photometric $-F(V)$ and spectral $F(s p)$ fluxes.

Shapovalova et al. (2004) ${ }^{1}$. This method allowed us to obtain a homogeneous set of spectra with the same wavelength calibration and the same [OIII] $\lambda 5007$ flux. Red spectra were scaled to the constant flux value of the narrow emission line [OI] $\lambda 6300$, by also applying the modified method of Van Groningen \& Wanders (1992) (see also Shapovalova et al. 2004). As a reference, we used a red spectrum obtained with the GHAO $2.1 \mathrm{~m}$ telescope during a good photometric night, and accurately scaled using the [OIII] $\lambda 5007$ line (details of the procedure are given in Shapovalova et al. 2008).

\subsection{Unification of the spectral data}

To investigate the long-term spectral variability of an AGN, it is necessary to gather a consistent set of spectral data. This requires us, in turn, to correct the line and continuum fluxes for aperture effects (Peterson \& Collins 1983), a detailed justification of which is given in Peterson et al. (1995), and will not be repeated here.

The NLR in 3C 390.3 is more compact than for most Sy1 galaxies. In narrow-band [OIII] images, the object displays compact nuclear emission without any extended structure (Baum et al. 1988). The results of panoramic two-dimensional spectrophotometry of the nuclear region of this object imply that the [OIII] $\lambda 5007$ emission originates in a zone smaller than $r<2$ arsec (Bochkarev et al. 1997). Furthermore, Osterbrock et al. (1975) obtained a rather low value of the [OIII] $F(\lambda 4363) / F(\lambda 5007)$ line ratio, implying a moderately high electron density in the NLR (several $10^{6} \mathrm{~cm}^{-3}$ ). Therefore, the NLR in 3C 390.3 can be considered to be a point source, and we did not correct for aperture effects either the ratio of nonstellar continuum to narrow-line flux or the ratio of the broad to narrow line flux, as the light losses in the slit are similar for these components. In addition, our apertures are $2^{\prime \prime} \times 6^{\prime \prime}$ or $2.5^{\prime \prime} \times 6$ ", which are greater than the size of the NLR. However, the light contribution to the continuum from the host galaxy does depend on the aperture, and it is necessary to introduce the

${ }^{1}$ See Appendix A of Shapovalova et al. (2004). corresponding corrections. To accomplish this goal, we adopted the relation (see Peterson et al. 1995)

$F_{\text {con }}=F(4959+5007)\left[\frac{F_{\text {con }}}{F(4959+5007)}\right]_{\mathrm{obs}}-G$,

where $F(4959+5007)$ is the absolute flux in the [OIII] doublet and the value in brackets is the continuum to [OIII] line observed flux ratio, where $G$ is an aperture-dependent correction factor that accounts for the host galaxy flux. Since most of our spectra were obtained with the $6 \mathrm{~m}$ telescope through an aperture of $2^{\prime \prime} \times$ $6^{\prime \prime}$, this instrumental setup was adopted as standard (i.e. $G=0$ by definition). The value of $G$ for the spectra obtained at the $2.1 \mathrm{~m}$ telescope (aperture of $2.5^{\prime \prime} \times 6.0^{\prime \prime}$ ) is $G=0.046$, while for those obtained at the $1 \mathrm{~m}$ telescope (aperture of $8^{\prime \prime} \times 19.8^{\prime \prime}$ ) $G=0.321 \pm 0.028$.

We note here that there is the influence of seeing on $G$ (Sergeev et al. 2002). We estimated that if the seeing changes from $1^{\prime \prime}$ to $3^{\prime \prime}$ (that was typical for our observations), the $G$ would change at the level of $\sim 5 \%$ (see Sergeev et al. 2002). For standard apertures $2^{\prime \prime} \times 6^{\prime \prime}$, and another aperture $2.5^{\prime \prime} \times 6^{\prime \prime}$ that is also very close to the standard one, the estimated $G$ is very small $\left(G \sim 0.046 \times 10^{-15} \mathrm{erg} \mathrm{cm}^{-2} \mathrm{~s}^{-1} \mathrm{~A}^{-1}\right.$ for aperture $2.5^{\prime \prime} \times 6^{\prime \prime}$, or $3 \%$ of the minimal flux). Therefore, in our case, the seeing has a small influence on $G$.

We did not estimate the contribution of the host galaxy, since it is constant and does not influence the analysis of variability, but we mention here that Sergeev et al. (2002) estimated that the host galaxy contributions (for a seeing of 2.5") in the $\mathrm{H} \alpha$ and $\mathrm{H} \beta$ wavelength region are $\sim 7 \times 10^{-16} \mathrm{erg} \mathrm{cm}^{-2} \mathrm{~s}^{-1} \mathrm{~A}^{-1}$, and $\sim 4.6 \times 10^{-16} \mathrm{erg} \mathrm{cm}^{-2} \mathrm{~s}^{-1} \mathrm{~A}^{-1}$, respectively.

\subsection{The narrow emission-line template}

To obtain only the broad component of Balmer lines, i.e. to remove the narrow lines, we created a narrow line template using the blue and red spectra in the minimum activity state (Sep. 09, 1997 and Feb. 21, 2002), obtained with a spectral resolution of $\sim 8 \AA$. In these spectra, the broad $\mathrm{H} \beta$ component was very weak, and the broad component of the higher Balmer line series were absent. To construct the narrow line template for the $\mathrm{H} \beta$ wavelength band, we first estimated the broad component (as can be seen in Fig. 2, top) and then subtracted it from the composite spectrum. The narrow line template is shown in Fig. 2 (bottom).

In the case of $\mathrm{H} \alpha$, there is a problem of removing the atmospheric $B$-band $(\mathrm{O} 2)$ at $\lambda=6870 \AA$ in the blue wing. In comparison stars, the $\mathrm{B}$ band is very weak due to the shortness of the exposures, therefore it is not possible to remove it using the relative flux calibration. To remove this component, we used the spectra of NGC 4151, taken with long exposures in nights closeby in time. Using different coefficients, we then subtracted the $B$-band from the $\mathrm{H} \alpha$ observed spectrum in the minimum activity state (Sep. 9, 1997). Using this spectrum, we fitted the broad component by the spline method (Fig. 3, panel A). The narrow line template for the $\mathrm{H} \alpha$ line was obtained by subtracting this broad component from the observed spectrum (Fig. 3, panel A).

In addition, we apply the Gaussian-fitting method to the $\mathrm{H} \alpha$ wavelength region, after subtracting the $B$-band (see Fig. 3, panel B). The narrow components are narrow $\mathrm{H} \alpha,[\mathrm{N} \mathrm{II}] \lambda \lambda$ 6548,6584, [O I] $\lambda \lambda$ 6300,6364) [S II] $\lambda \lambda$ 6717,6731, while the broad line contains 3 components, the broad blue, core, and the broad red Gaussians (panel B, bottom). In panel B (up) of Fig. 3, the observed spectrum and the sum of all Gaussian components and their residuals are presented. One can see that 

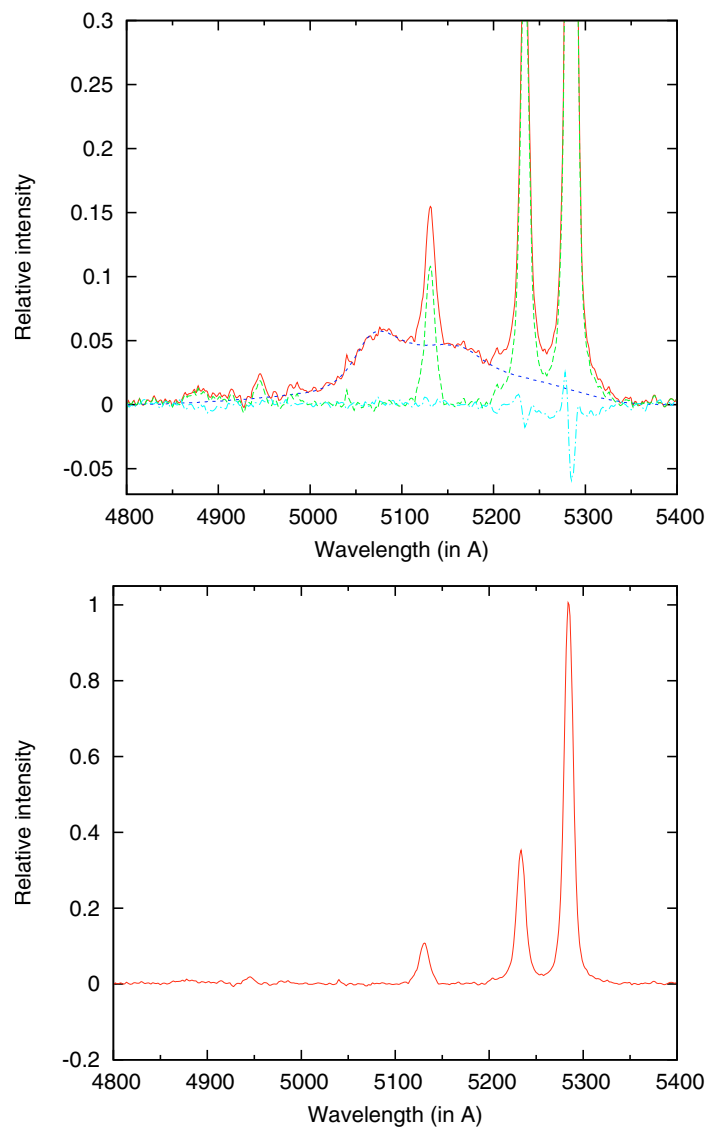

Fig. 2. An example of the narrow line template for the $\mathrm{H} \beta$ line (bottom) obtained by subtracting from the observed spectrum a spline fit to the broad component (top).

the sum of Gaussian components closely represents the $\mathrm{H} \alpha$ observed profile, i.e. the residual is very small. In Fig. 3 (panel B, middle), we present the estimated broad component of $\mathrm{H} \alpha$, obtained by subtraction of sum of narrow Gaussian components, from the observed spectrum (after removing $B$-band).

We also compare the broad components, obtained by using the spline method and the Gaussian-fitting method (Fig. 3, panel $C$ ). As can be seen from Fig. 3 (panel C), there is practically no difference between the broad component obtained with these two different procedures.

\subsection{Measurements of the spectra and errors}

From the scaled spectra, we determined the average flux in the continuum at the observed wavelength $\sim 5384 \AA$ (or at $\sim 5100 \AA$ in the rest frame of $3 C 390.3, z=0.0556$ ) by means of flux averages in the frame of 5369-5399 $\AA$. To determine the observed fluxes of the broad $\mathrm{H} \beta$ and $\mathrm{H} \alpha$ lines, it is necessary to subtract the continuum. To achieve this goal, after subtracting the narrow components, a linear continuum was fitted at windows of $30 \AA$ located at $4800 \AA$ and $5400 \AA$ for the $\mathrm{H} \beta$ wavelength band, and at $6560 \AA$ and $7250 \AA$ for the $\mathrm{H} \alpha$ wavelength band.

After the continuum subtraction, we defined the observed fluxes in the lines in the wavelength intervals $4909-5353 \AA$ for $\mathrm{H} \beta$ and $6740-7160 \AA$ for $\mathrm{H} \alpha$.

In Table 2 (available electronically only), the fluxes for the continuum, broad $\mathrm{H} \alpha$, and $\mathrm{H} \beta$ lines are given. The mean error (uncertainty) in the fluxes is for the continuum $\approx 3 \%$, for the broad $\mathrm{H} \beta$ line $\approx 5 \%$ and the broad $\mathrm{H} \alpha$ line $\approx 10 \%$ (see
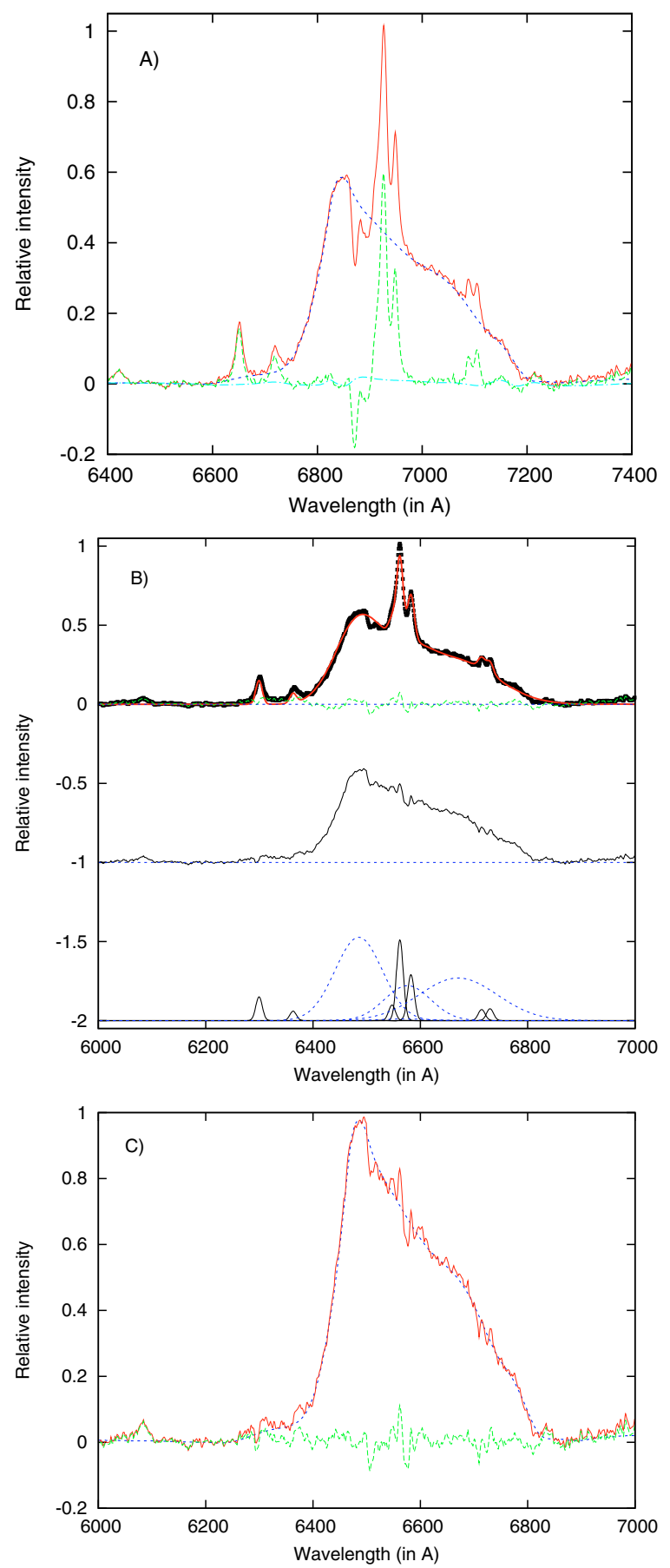

Fig. 3. An example of the substraction of the narrow lines in $\mathrm{H} \alpha$ using two different methods: (panel $\mathbf{A})$ ) the observed spectrum (continuous line), the mean broad component (dashed line) and the $\mathrm{H} \alpha$-narrow-linetemplate (dotted line); (panel B)) the observed spectrum and the sum of all Gaussians, as well as their residual (top); the broad component after subtraction of the sum of narrow Gaussians from the observed spectrum (middle); all Gaussian components (bottom); (panel C)) comparison of the profiles of the broad $\mathrm{H} \alpha$ line obtained by two different methods: the spline (dashed line), the Gaussian-fitting (full line), and the difference between these broad components (dash-dotted line at the bottom).

Table 3). These quantities were estimated by comparing results from spectra obtained within a time interval shorter than 3 days. In addition, we measured the line-part fluxes. To do that, we divided the $\mathrm{H} \alpha$ and $\mathrm{H} \beta$ profiles into three parts: the blue wing (covering the blue peak), the core, and the red wing. 
Table 3. The estimated mean flux errors of the $\mathrm{H} \alpha$ and $\mathrm{H} \beta$ lines and their segments.

\begin{tabular}{lcc}
\hline \hline Line & $\begin{array}{c}\text { Region } \\
{[\AA]}\end{array}$ & $\begin{array}{c}\sigma \pm e \\
{[\%]}\end{array}$ \\
\hline $\mathrm{H} \beta$ & $4909-5353$ & $4.3 \pm 2.4$ \\
$\mathrm{H} \alpha$ & $6740-7160$ & $8.4 \pm 6.3$ \\
Line wings and core & & \\
$\mathrm{H} \beta$-blue wing & $4977-5097$ & $4.2 \pm 3.0$ \\
$\mathrm{H} \beta$-core & $5097-5165$ & $3.8 \pm 2.6$ \\
$\mathrm{H} \beta$-red wing & $5165-5268$ & $4.8 \pm 3.0$ \\
$\mathrm{H} \alpha$-blue wing & $6720-6882$ & $7.2 \pm 5.6$ \\
$\mathrm{H} \alpha$-core & $6882-6974$ & $10.5 \pm 8.2$ \\
$\mathrm{H} \alpha$-red wing & $6974-7136$ & $7.9 \pm 5.4$ \\
Blue and red part of line & & \\
$\mathrm{H} \beta$-blue part & $4977-5131$ & $4.0 \pm 2.9$ \\
$\mathrm{H} \beta$-red part & $5131-5268$ & $4.3 \pm 2.8$ \\
$\mathrm{H} \alpha$-blue part & $6720-6928$ & $7.8 \pm 6.2$ \\
$\mathrm{H} \alpha$-red part & $6928-7136$ & $8.8 \pm 6.4$ \\
\hline
\end{tabular}

The intervals adopted were: (i) for $\mathrm{H} \beta$, a blue interval of -9004:-1988 km s${ }^{-1}(4977 \AA-5097 \AA)$, a central interval of $-1988:+1988 \mathrm{~km} \mathrm{~s}^{-1}(5097 \AA-5165 \AA)$, and a red interval of $+1988:+8010 \mathrm{~km} \mathrm{~s}^{-1}(5165 \AA-5268 \AA)$; (ii) for $\mathrm{H} \alpha$, a blue interval of $-9007:-1992 \mathrm{~km} \mathrm{~s}^{-1}(6720 \AA-6882 \AA)$, a central interval of $-1992:+1992 \mathrm{~km} \mathrm{~s}^{-1}(6882 \AA-6974 \AA)$, and a red interval of $+1992:+9007 \mathrm{~km} \mathrm{~s}^{-1}(6974 \AA-7136 \AA)$.

In addition, we measured only the blue and red part of lines by considering the intervals $-9004: 0:+8010 \mathrm{~km} \mathrm{~s}^{-1}$ for $\mathrm{H} \beta$ and -9007:0:+9007 $\mathrm{km} \mathrm{s}^{-1}$ for $\mathrm{H} \alpha$.

In Table 3, we present the estimated mean flux errors in the $\mathrm{H} \alpha$ and $\mathrm{H} \beta$ lines and their segments. Figure 4 shows the distribution of the flux error in the $\mathrm{H} \beta$ line parts against the corresponding fluxes. There is only a weak regression between the flux error and flux in the broad $\mathrm{H} \beta$ line, while we can not detect the regression for the case of the broad $\mathrm{H} \alpha$ line.

\section{Data analysis}

We analyzed variations in the continuum and lines using a total of 129 spectra covering the $\mathrm{H} \beta$ wavelength region, and 48 spectra covering the $\mathrm{H} \alpha$ line. During the monitoring period, we found that the minimum in the continuum flux occurred during August-September 1997, and the maximum in May-June 2007. In Fig. 5, the shape of 3C 390.3 spectra around the minimum and maximum of activity is presented. As can be seen from Fig. 5, the slope of the continuum in the blue part of the spectrum in the minimum-activity state was significantly flatter than in the high-activity state. The wings of $\mathrm{H} \beta$ and $\mathrm{H} \alpha$ became extremely weak in the minimum state, and those of $\mathrm{H} \gamma$ and higher Balmer line series could not be detected at all. These profiles correspond to the Sy 1.8 type and not to Sy 1, as this AGN usually is classified. The spectral type of this object therefore changes with time as noted earlier. In April 1984, the nucleus of 3C 390.3 experienced a very deep minimum, the broad wings of hydrogen lines became much weaker (they almost completely vanished in April 1984) and the spectrum of the nucleus was identified as the Sy 2 (Penston \& Perez 1984). Therefore, to compare the continuum and broad line variability we use the broad $\mathrm{H} \alpha$ and $\mathrm{H} \beta$ lines only, and the measured continuum around $\lambda 5100 \AA$ (rest frame). Our measurements are given in Table 2 (available electronically only).

\subsection{Variability of the emission lines and the optical continuum}

As noted above (see e.g. Paper I, Dietrich et al. 1998; Sergeev et al. 2002; Tao et al. 2008, etc.), the optical flux variability of 3C 390.3 in the line as well as in the continuum was observed. The light curves of the broad $\mathrm{H} \alpha, \mathrm{H} \beta$ line, and continuum fluxes are illustrated in Fig. 6. In general, the light curve of the continuum is similar to that of $\mathrm{H} \beta$ and $\mathrm{H} \alpha$, but there are some differences in the sharpness of the peaks (maxima) and also it is obvious that a lag exists between the continuum and $\mathrm{H} \beta$ and $\mathrm{H} \alpha$ light curves.

The maximum amplitude of the flux variations during a monitoring period (1995-2007) corresponds to factors of $\sim 5.2$ for the continuum (at $\sim 5100 \AA$ in the rest frame), $\sim 4.7$ for $\mathrm{H} \beta$, and $\sim 3.4$ for $\mathrm{H} \alpha$ (see in Sect. 3.5, Table 5). As one can see in Fig. 6, there are several maxima (outbursts). They are more prominent in the continuum light curve than in the $\mathrm{H} \beta$ and $\mathrm{H} \alpha$ light curves. In 1995-2002, an outburst of decreasing amplitude and duration (intervals between minima) from 1000 days to $\sim 400$ days can be seen. In 2003-2007, we can discern about $\sim 3$ outbursts on ascending branch of curves of durations $\sim 600$ days, $\sim 900$ days, $\sim 500$ days, and a maximum in 2007 . The similar outbursts (oscillations) in the light curve in $B$ band from 1995 to 2001 are also clearly seen (see Fig. 2 in Paper I). These minima/maxima may be indicative of a periodicity (or quasi-periodical oscillation) in the continuum and broad-line light curves.

\subsection{Light curve analysis}

Searching for periodicity has been an important part of variability studies of AGN, because the confirmed periodicity would strongly constrain possible physical models and help us to determine the relevant physical parameters of AGN. We therefore applied a wavelet transform with the Morlet wavelet to see if there is periodicity in the continuum light curve. Wavelet analysis involves a transform from one-dimensional time series to a diffuse two-dimensional time-frequency image for detecting localized (pseudo-)periodic fluctuations from subsets of the time series corresponding to a limited time span (Torrence \& Compo 1998).

We employed the standard wavelet IDL codes of Torrence \& Compo (1998) to look for periodicities in the 3C 390.3 opticalcontinuum and $\mathrm{H} \beta$ flux light curves. A Morlet wavelet is particularly suited to the analysis of time series and has been successfully applied to study variability in AGN (i.e. Lachowicz et al. 2009; Hovatta 2008; Gupta et al. 2009, etc.). We therefore employ a Morlet wavelet here, which is defined as

$\Psi_{\mathrm{t}}(s)=\pi^{-1 / 4} \mathrm{e}^{\mathrm{i} k t} \mathrm{e}^{\frac{-t^{2}}{2 s^{2}}}$

where $t$ is the time parameter, $\pi^{-1 / 4}$ is the normalization factor, and $s, k$ are wavelet scale and oscillation frequency parameter, respectively. The parameter $k$ has been set equal to 6 to satisfy the admissibility condition.

The continuous wavelet power spectra is subject to edge artefacts because the wavelet is not completely localized in time. It is useful to introduce a cone of influence (COI) in which the transform suffers from these edge effects (Torrence \& Compo 1998). Periods inside the COI are subject to edge effects and might be dubious.

Figure 7 shows the continuous wavelet power spectra of the long-term light curve in the optical-continuum and $\mathrm{H} \beta$ fluxes, respectively. There are evidently common features in the wavelet 

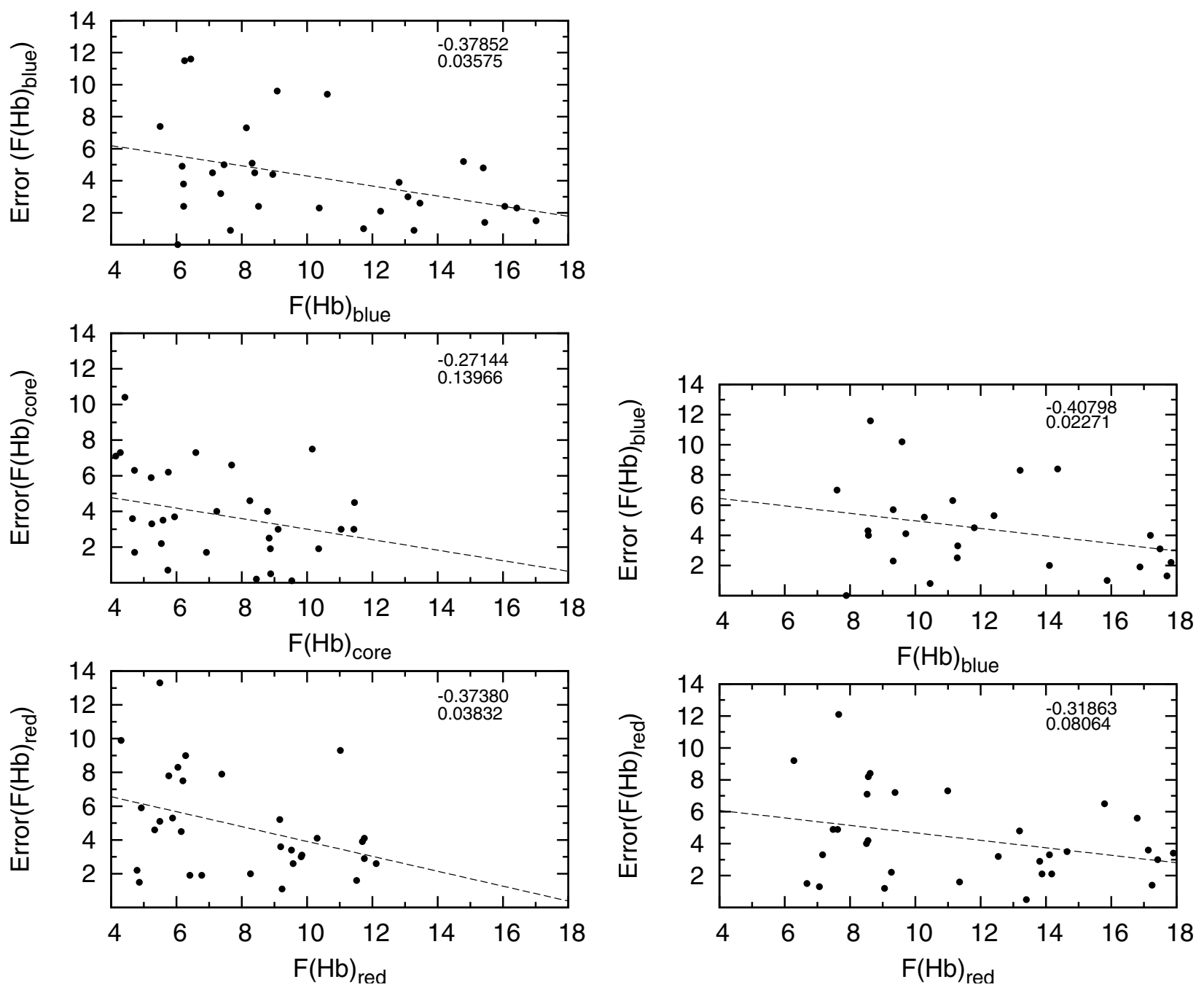

Fig. 4. The estimated errors in the flux measurements as functions of the corresponding flux, in case we divided lines in 3 parts (left panels) and 2 parts (right panels). The correlation coefficient and the $p$-value are given in the upper right corner.

power of the two time series. Both wavelet spectra have a longterm periodicity $(\sim 1.65-2.45 \mathrm{yr})$ in the late part of the light curve, above the $95 \%$ confidence level, but within the COI (periodicity being too close to the total signal length).

However, we found a varying timescale of $0.5-0.74 \mathrm{yr}$ around 2004 with a confidence of $>99.5 \%$ that this component does not arise by chance (significance computed assuming the global wavelet spectrum as the theoretical background spectrum following Torrence \& Compo 1998) and located above the COI.

Taking into account that this method provides some indication about periodicity, we performed an analysis assuming that there can be several reasons for variability, e.g. some kinds of shocks or flares in the accretion disk. We therefore assumed that the total flux in the line and continuum can be represented as

$F_{\text {tot }}^{\mathrm{c}, \mathrm{l}}(t)=F_{\mathrm{A}}^{\mathrm{c}, \mathrm{l}}(t)+F_{\mathrm{B}}^{\mathrm{c}, \mathrm{l}}(t) \cdot G^{\mathrm{c} .1}(t)$,

where $F_{\mathrm{A}, \mathrm{B}}^{\mathrm{c}, 1}$ are different components of the continuum/line fluxes, and $G^{\text {c.l }}$ is the scaling function. All these functions may vary with time.

Using the above equation and observed light curves of the continuum and $\mathrm{H} \beta$ line, we estimated $F_{\mathrm{A}}(t)$ by assuming this function to be an envelope covering minima on the light curves, and $F_{\mathrm{B}}(t)$ to be an envelope covering maxima on the light curves. The illustration of the decomposition of the continuum and $\mathrm{H} \beta$ light curves into three aforementioned functions are given in Figs. 8 and 9.

In Fig. 10 we compare the (rescaled) function $F_{\mathrm{A}}, F_{\mathrm{B}}$, and $\mathrm{G}$ estimated from the continuum (dashed line, full circles) and $\mathrm{H} \beta$ (dotted line, asterisks) light curve. As one can see (Fig. 10, first panel), the shapes of $F_{\mathrm{A}}$ seem to be similar with slight differences, which may be caused by estimation of minima. In contrast, the shapes of $F_{\mathrm{B}}$ are different (second panel), which is indicative of a rapidly increasing continuum $F_{\mathrm{B}}$ from 2002, when higher activity of AGN also started. The $F_{\mathrm{B}}$ component may be quasi-periodical in both the continuum and $\mathrm{H} \beta$, i.e. it may contain two peaks separated by about ten years, that 10-year signal is also visible in the wavelet plot, though in the COI. Finally, the $G(t)$ exhibits (third and fourth panel) quasi-periodical oscillations with a period of around 2-4 yr, which is very close to the period indicated by Morlet wavelet spectral analysis.

The minima and maxima of the continuum $G(t)$ more closely match those of the $\mathrm{H} \beta G(t)$ after a shift of $\sim 95$ days, which is close to the lag obtained from the CCF analysis (see Sect. 3.6). It is also interesting that quasi-periodical oscillations can be seen until 2002, before the beginning of the high activity phase (from 2002 to 2007). In the period 1995-2002, there are three prominent maxima. From 2002 to 2004, at the beginning of the activity, a clear peak cannot be seen, while from 2004 to 2007 two maxima can be recognized (see Fig. 10, 3rd and 4th panels). 
A. I. Shapovalova et al.: Spectral monitoring of 3C 390.3. I.

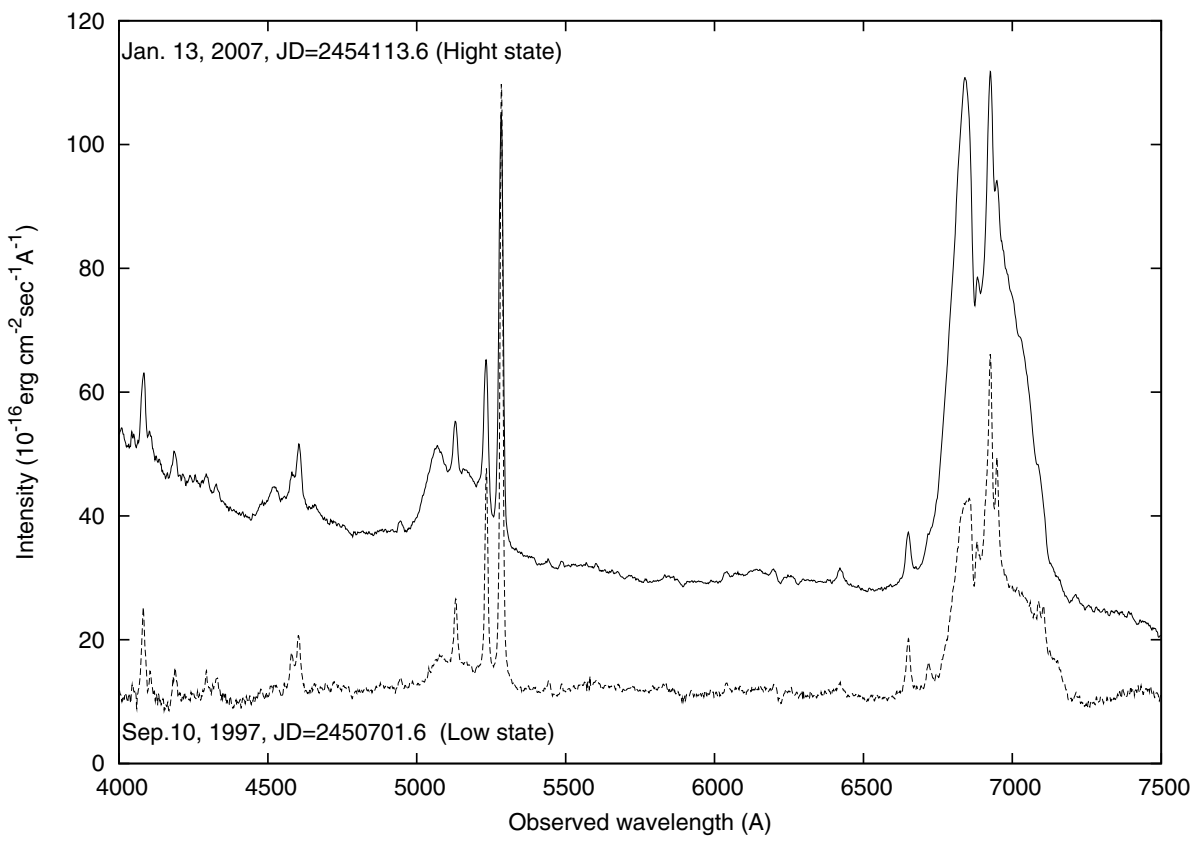

Fig. 5. The spectra of $3 \mathrm{C} 390.3$ close to maximum (up - observed in 2007) and minimum (bottom-observed in 1997).

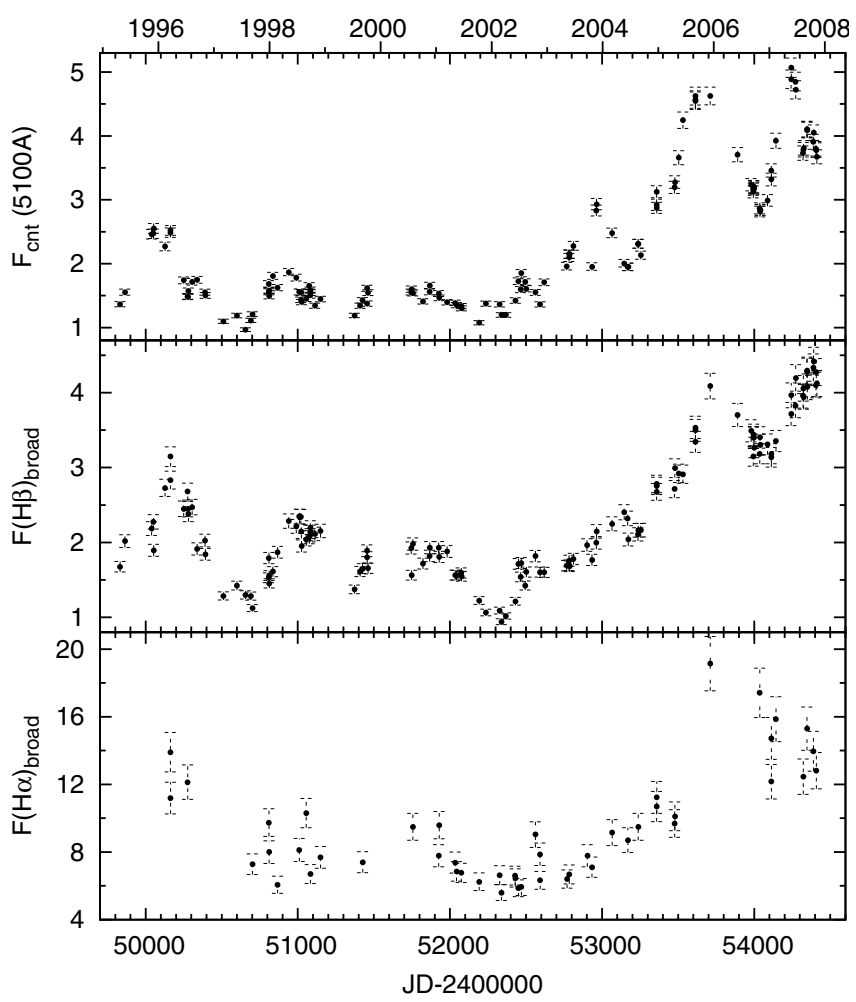

Fig. 6. Light curves of the continuum (top), broad $\mathrm{H} \beta$ (middle), and $\mathrm{H} \alpha$ (bottom). The continuum flux is given in $10^{-15} \mathrm{erg} \mathrm{cm}^{-2} \mathrm{~s}^{-1} \AA^{-1}$, and $\mathrm{H} \beta$ and $\mathrm{H} \alpha$ line in $10^{-13} \mathrm{erg} \mathrm{cm}^{-2} \mathrm{~s}^{-1}$.

\subsection{Changes in the broad line profiles}

We first inspected the $\mathrm{H} \alpha$ and $\mathrm{H} \beta$ profiles using spectra with a resolution of $8 \AA$ for different epochs, comparing $\mathrm{H} \alpha$ and $\mathrm{H} \beta$ profiles. As one can see in Fig. 11, there are no large differences between the line profiles of $\mathrm{H} \alpha$ and $\mathrm{H} \beta$. The lines in all considered epochs show a blue-boosted and red-tailed peaks, which are common for emission from a relativistic disk. However, there are some differences among the $\mathrm{H} \alpha$ and $\mathrm{H} \beta$ line profiles that vary during the monitoring period. For instance, $\mathrm{H} \beta$ exhibits a bump
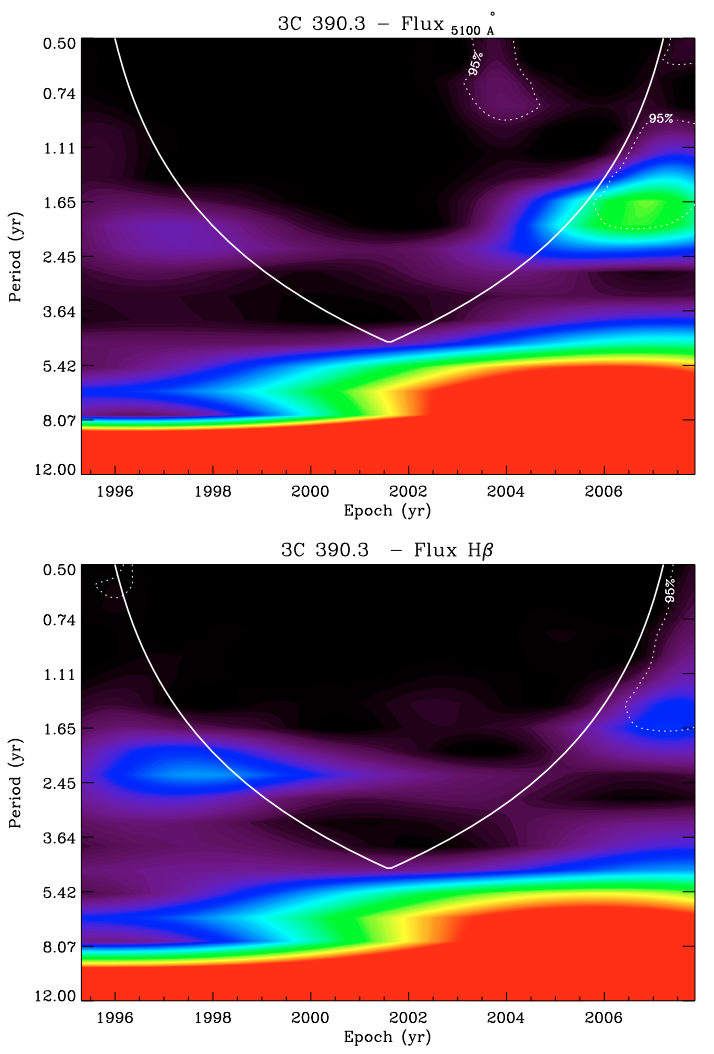

Fig. 7. The continuous wavelet power spectra of the long-term light curve of the optical continuum at $5100 \AA$ (top) and in the $\mathrm{H} \beta$ (bottom) fluxes. The region below the thick dashed white line indicates the COI where edge effects resulting from the finite length of the time series, become important. Therefore, periods inside the COI might be dubious. The dashed contours indicate the 95 per cent confidence level. Redder colors corresponding to stronger signals.

in its central part during 1997-1998, which later disappears (until 2002). From the minimal phase of activity in 2002, the red wing remains stronger in $\mathrm{H} \beta$ than in $\mathrm{H} \alpha$. During maximum activity, between 2004 and 2007, the $\mathrm{H} \beta$ is also broader than $\mathrm{H} \alpha$. 

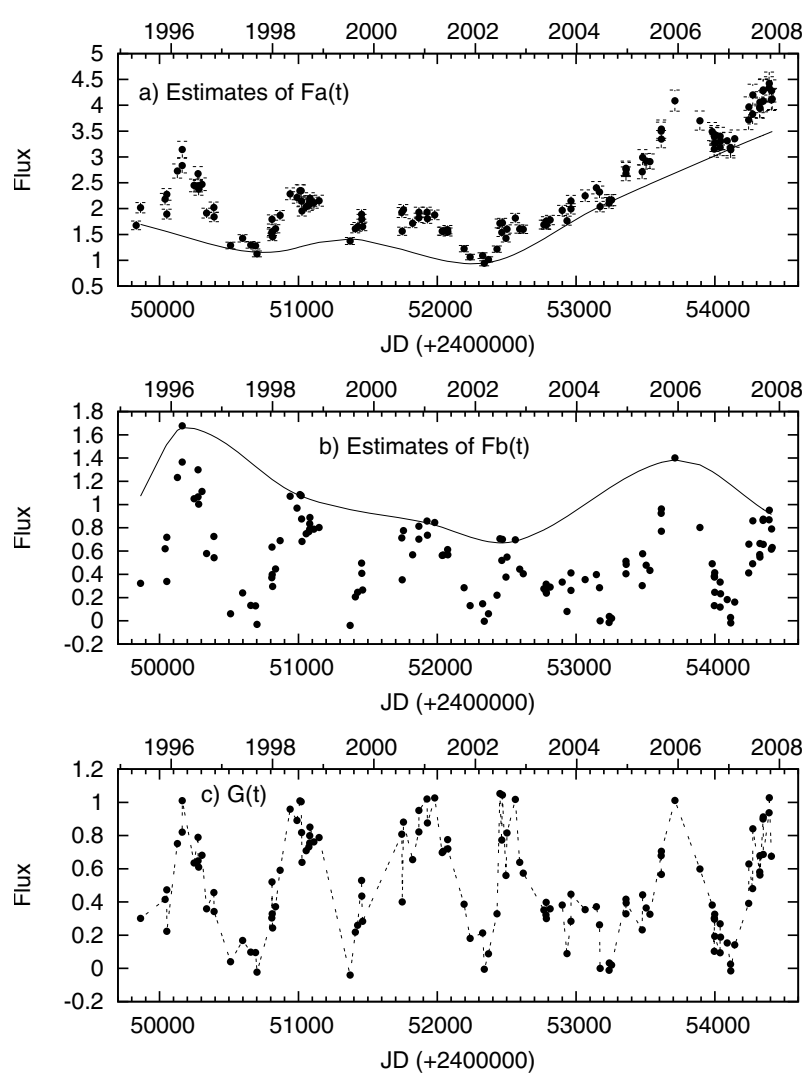

Fig. 8. The decomposition of the continuum light curve: a) the flux in the continuum and estimation of $F_{\mathrm{A}}(t)$ component, b) the estimation of $F_{\mathrm{B}}(t)$ component, $\left.\mathbf{c}\right)$ the scaling factor $G(t)$ as an indicator of the quasi-periodical variability. The total flux, $F_{\mathrm{A}}$, and $F_{\mathrm{B}}$ are given in $10^{-15} \mathrm{erg} \mathrm{cm}^{-2} \mathrm{~s}^{-1} \AA^{-1}$.
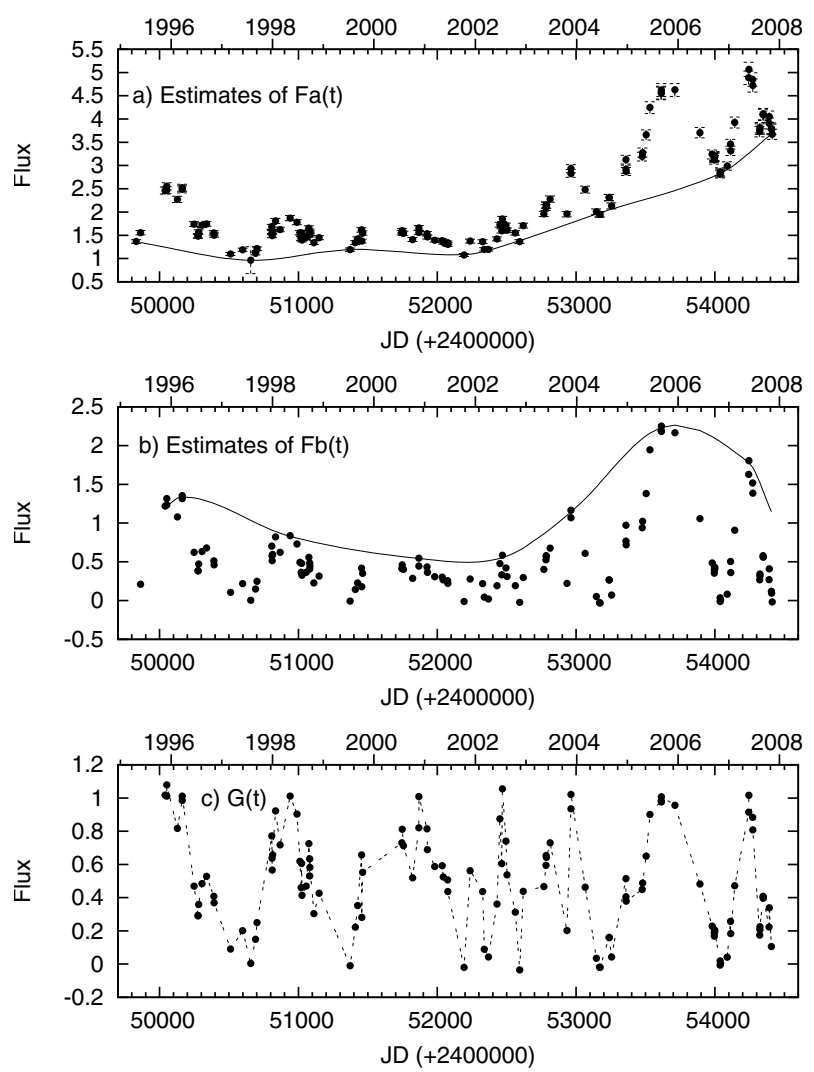

Fig. 9. The same as in Fig. 8, but for the $\mathrm{H} \beta$ line. The total flux, $F_{\mathrm{A}}$, and $F_{\mathrm{B}}$ are given in $10^{-13} \mathrm{erg} \mathrm{cm}^{-2} \mathrm{~s}^{-1}$.
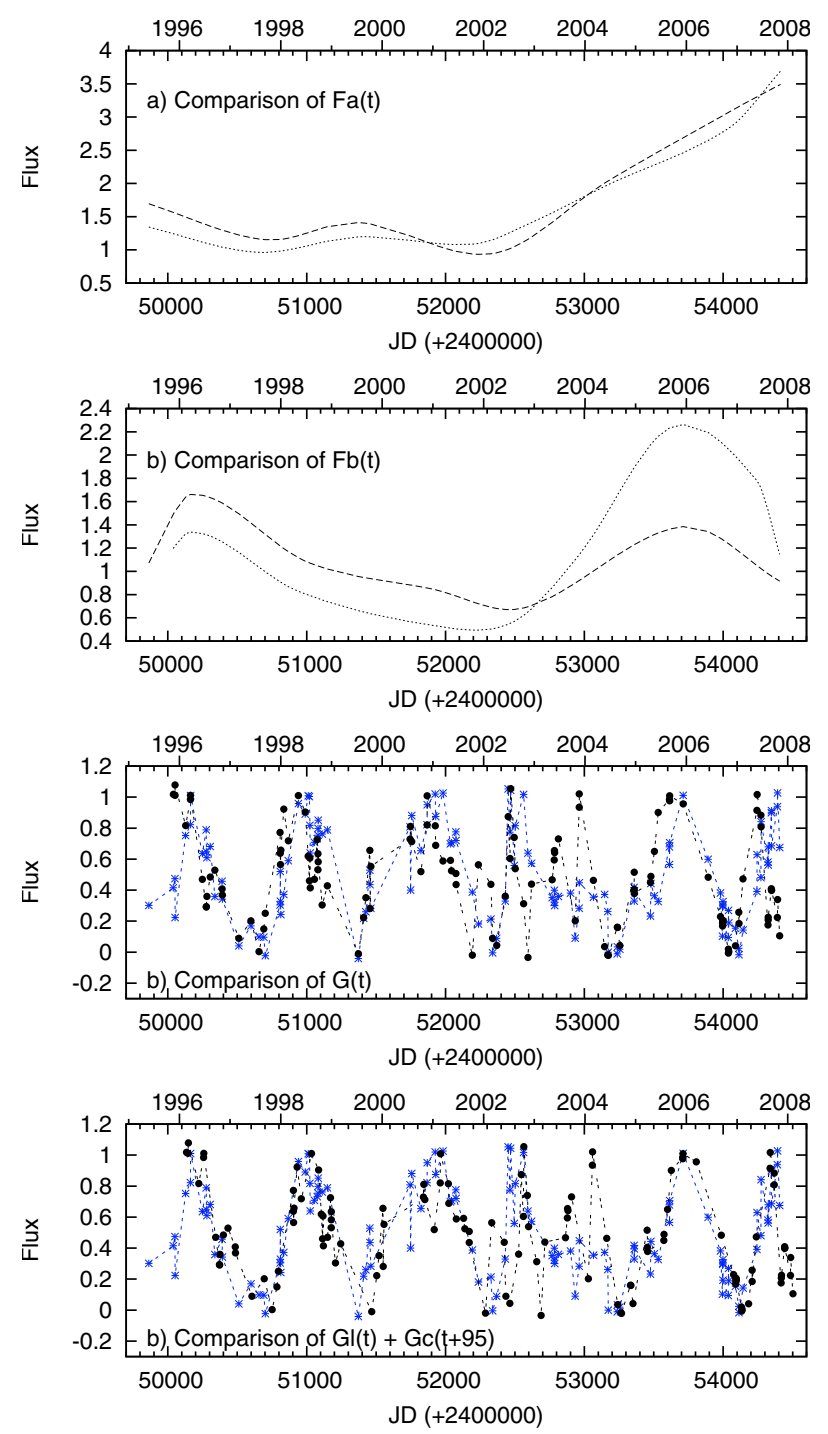

Fig. 10. The comparison of different flux components of $\mathrm{H} \beta$ (dotted line) and continuum (dashed line). Two last panels present comparison of $G(t)$, where last one is taken to have $G(t+95)$ for the continuum. Asterisks stands for $\mathrm{H} \beta$ and full circles for the continuum.

As noted above, the first inspection of line profiles identified differences between $\mathrm{H} \alpha$ and $\mathrm{H} \beta$ line shapes after the beginning of the phase of activity in 2002. We therefore, decided to consider the mean profiles of $\mathrm{H} \alpha$ and $\mathrm{H} \beta$ during the whole monitoring period, and both the mean and root-mean-square (rms) profiles for the period before March 05, 2002 (period I, JD 2452339.01 according the minimum in $\mathrm{H} \beta$ ) and after that (period II, see Figs. 12). The mean and rms profiles of both lines have "double-peaked" structure and a full width at zero intensity of about $20000 \mathrm{~km} \mathrm{~s}^{-1}$. The blue bump in the mean spectrum is located at a radial velocity of about $-4000 \mathrm{~km} \mathrm{~s}^{-1}$ relative to the $\mathrm{H} \alpha$ and $\mathrm{H} \beta$ narrow components, while the red bump with the flatter peak is located between +2000 and $+5000 \mathrm{~km} \mathrm{~s}^{-1}$. The blue bump in the mean profiles is brighter than the red one during 1995-2007. The rms profiles show that the variability of the blue wing is stronger than that of the red (in all periods). As can also be seen in Fig. 12, in the first period a central component was present and apart from the variation in two peaks, there is also a dominant variation in the core of the lines. We note that the sharp narrow central peak in the $\mathrm{H} \alpha \mathrm{rms}$ and $\mathrm{H} \beta \mathrm{rms}$ may be caused by the narrow line substraction. 
A. I. Shapovalova et al.: Spectral monitoring of 3C 390.3. I.

Table 4. The line widths and asymmetry measurements for the whole monitoring period, period I and II.

\begin{tabular}{lccccc}
\hline \hline Spectrum & $\begin{array}{c}F W H M \\
\mathrm{~km} \mathrm{~s}^{-1}\end{array}$ & $\mathrm{~A}$ & $\begin{array}{c}\mathrm{BP} \\
\mathrm{km} \mathrm{s}^{-1}\end{array}$ & $\begin{array}{c}\mathrm{RP} \\
\mathrm{km} \mathrm{s}^{-1}\end{array}$ & $\mathrm{Ib} / \mathrm{Ir}$ \\
\hline $\mathrm{H} \alpha_{\text {mean }}$ & $10992 \pm 499$ & $0.85 \pm 0.05$ & $-3709 \pm 60$ & $2661 \pm 240$ & $1.65 \pm 0.03$ \\
$\mathrm{H} \alpha_{\text {rms }}$ & $9866 \pm 723$ & $0.63 \pm 0.09$ & $-3807 \pm 130$ & $1442 \pm 80$ & $1.52 \pm 0.05$ \\
$\mathrm{H} \beta_{\text {mean }}$ & $11918 \pm 325$ & $0.94 \pm 0.00$ & $-3601 \pm 40$ & $2154 \pm 70$ & $1.32 \pm 0.02$ \\
$\mathrm{H} \beta_{\text {rms }}$ & $12093 \pm 325$ & $0.82 \pm 0.02$ & $-3772 \pm 40$ & $2150 \pm 70$ & $1.34 \pm 0.02$ \\
$\mathrm{H} \alpha_{\text {mean }}$ I & $11526 \pm 393$ & $0.97 \pm 0.03$ & $-3704 \pm 20$ & $3178 \pm 180$ & $1.65 \pm 0.01$ \\
$\mathrm{H} \alpha_{\text {rms I }}$ & $10299 \pm 149$ & $1.08 \pm 0.00$ & $-3632 \pm 50$ & $4117 \pm 30$ & $1.02 \pm 0.01$ \\
$\mathrm{H} \beta_{\text {mean }}$ I & $11957 \pm 504$ & $1.05 \pm 0.07$ & $-3453 \pm 210$ & $2947 \pm 770$ & $1.41 \pm 0.04$ \\
$\mathrm{H} \beta_{\text {rms }}$ I & $12561 \pm 610$ & $1.10 \pm 0.09$ & $-3408 \pm 370$ & $4063 \pm 20$ & $1.12 \pm 0.01$ \\
$\mathrm{H} \alpha_{\text {mean }}$ II & $10516 \pm 822$ & $0.75 \pm 0.10$ & $-3687 \pm 10$ & $2213 \pm 530$ & $1.64 \pm 0.02$ \\
$\mathrm{H} \alpha_{\text {rms }}$ II & $9362 \pm 564$ & $0.57 \pm 0.06$ & $-3816 \pm 110$ & $1450 \pm 80$ & $1.63 \pm 0.03$ \\
$\mathrm{H} \beta_{\text {mean }}$ II & $12015 \pm 205$ & $0.90 \pm 0.01$ & $-3608 \pm 60$ & $2151 \pm 70$ & $1.32 \pm 0.01$ \\
$\mathrm{H} \beta_{\text {rms }}$ II & $11373 \pm 264$ & $0.77 \pm 0.00$ & $-3834 \pm 120$ & $1671 \pm 190$ & $1.39 \pm 0.01$ \\
\hline
\end{tabular}

Notes. Column (1): the averaged and rms broad line profiles of $\mathrm{H} \alpha$ and $\mathrm{H} \beta$. Column (2): the measured full width at half maximum (FWHM). Column (3): the corresponding asymmetry A. Column (3): the position of the blue peak. Column (4): the position of the red peak. Column (5): the ratio of the blue to red peak flux.

We measured the full width at half maximum $(F W H M)$ in the rms and averaged broad line profiles, and we defined an asymmetry parameter $\mathrm{A}$ as the ratio of the red to blue half width at half maximum $(H W H M)$, i.e. $A=H W H M_{\text {red }} / H W H M_{\text {blue }}$. We also estimated the peak positions and their ratios. The measured values for the broad $\mathrm{H} \beta$ and $\mathrm{H} \alpha$ lines and their rms values are given in Table 4 . As can be seen from Table 4, the FWHM of the mean $\mathrm{H} \beta$ line (as well as the rms) obtained from the whole monitoring period is broader than that of $\mathrm{H} \alpha$, and line (and rms) profiles exhibit the blue asymmetry on the half maximum.

There are also differences between line and rms parameters obtained from periods I and II. As one can see from Table 4, in period $\mathrm{I}$, the $F W H M$ s of $\mathrm{H} \beta$ and $\mathrm{H} \alpha$ are practically identical, while $\mathrm{H} \alpha$ exhibits a slightly blue and $\mathrm{H} \beta$ slightly red asymmetry (within the errorbars), i.e. they are almost symmetric at the half intensity maximum (here measured to be the blue peak maximum). In contrast, during the phase of flaring (2002-2007), the $F W H M$ of $\mathrm{H} \beta$ remains broader than $\mathrm{H} \alpha$ (around $1500 \mathrm{~km} \mathrm{~s}^{-1}$ ), and both lines have a significant blue asymmetry at half intensity maximum. On the other hand, the separation between peaks is higher in period I than in period II, which is indicative of some global perturbation in the BLR. This result agrees with that of Paper I, where it was found that the difference in the radial velocities of the blue and red peaks is anticorrelated with the brightness of $\mathrm{H} \beta$ and continuum. We note that the blue to red peak ratio does not differ too significantly between the two periods, but there is a difference in the peak ratios and their separations of the mean $\mathrm{H} \alpha\left(\mathrm{RP}-\mathrm{BP} \approx 6500 \mathrm{~km} \mathrm{~s}^{-1}, \mathrm{Ib} / \mathrm{Ir} \approx 1.65\right)$ and $\mathrm{H} \beta\left(\mathrm{RP}-\mathrm{BP} \approx 5600 \mathrm{~km} \mathrm{~s}^{-1}, \mathrm{Ib} / \mathrm{Ir} \approx 1.30\right)$ lines, which may reflect a stratification in the BLR, i.e. the different dimensions of the $\mathrm{H} \alpha$ and $\mathrm{H} \beta$ emitting regions.

\subsection{Flux variability in the wings and core of the broad $H \alpha$ and $H \beta$ lines and various correlations}

To determine whether there are any variations in the structure of the BLR (here probably in the disk geometry), we investigated the relationships between different parts of $\mathrm{H} \alpha$ and $\mathrm{H} \beta$.

Light curves for the wings and cores of $\mathrm{H} \alpha$ and $\mathrm{H} \beta$ are presented in Fig. 13. As can be seen, the flux in the wings and cores of both lines behaved similarly during the monitoring period. This was also found when we considered only the blue and red parts of the lines.
We found that the flux ratio of different $\mathrm{H} \alpha$ to $\mathrm{H} \beta$ parts varies during the monitoring period. In Fig. 14, we presented the flux ratios of the red to blue part (asterisk blue-line), the central to blue (circles black-line), and the red to blue for when we divided lines into only two parts (triangle red-line). As one can see from Fig. 14, the blue part remains more prominent in the phase of higher activity in the period 2002-2007. This also reflects some global changes in the line-emitting regions.

We also identified a correlation between the continuum flux and both the integral flux and flux of different parts of the line (see Fig. 15). In Fig. 16, we present the relationship between the $\mathrm{H} \alpha$ and $\mathrm{H} \beta$ line parts. As we see in Figs. 15 and 16, there is a linear relationship between the fluxes of the broad $\mathrm{H} \alpha$ and $\mathrm{H} \beta$ lines, their parts, and continuum fluxes (Fig. 15), as well between the broad line parts themselves (Fig. 16). These linear correlations indicate that for both lines, both the blue and red wings, and the core originate in the BLR that is ionized by the AGN source.

\subsection{Variability analysis}

In Table 5, we present various parameters characterizing the continuum, the integral line, and the line parts, where $F$ is the mean flux and $\sigma(F)$ is its standard deviation, $R(\max )$ is the ratio of the maximum to the minimum flux in the monitoring period, $F$ (var) is a measured (uncertainty-corrected) estimate of the variation amplitude with respect to the mean flux, defined by O'Brien et al. (1998) to be

$F(\operatorname{var})=\left[\sqrt{\sigma(F)^{2}-e^{2}}\right] / F($ mean $)$,

where $e^{2}$ is the mean square value of the individual measurement uncertainty for $N$ observations, i.e. $e^{2}=\frac{1}{N} \sum_{i}^{N} e(i)^{2}$. From Table 5 , one can see that the amplitude of variability $F$ (var) is larger in the continuum (0.46) than in the $\mathrm{H} \beta(0.38)$ and $\mathrm{H} \alpha$ (0.35) broad emission lines. The blue $\mathrm{H} \beta$ and $\mathrm{H} \alpha$ wings vary slightly more strongly than the red ones. The maximum to minimum flux ratio, $R(\max )$, was maximal in the continuum (5.2) and minimal in $\mathrm{H} \alpha$ (3.4) during the monitoring period. We note that fom the IUE observations performed between 31 December 1994 and 05 March 1996 (O'Brien et al. 1998), $F$ (var) was smaller for Ly $\alpha(0.14)$, CIV ( 0.3), and HeII 1640 (0.31) than in optical lines measured during the monitoring period 1995-2007. However, the optical continuum 


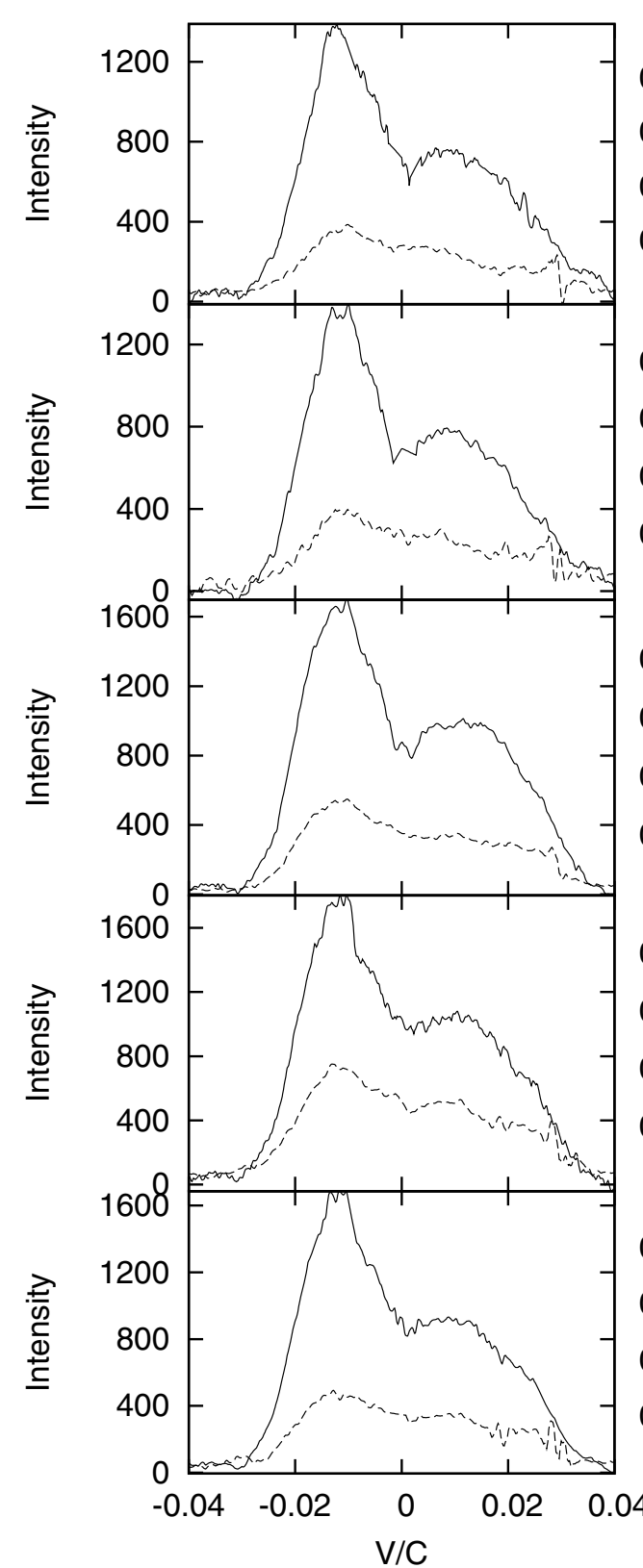

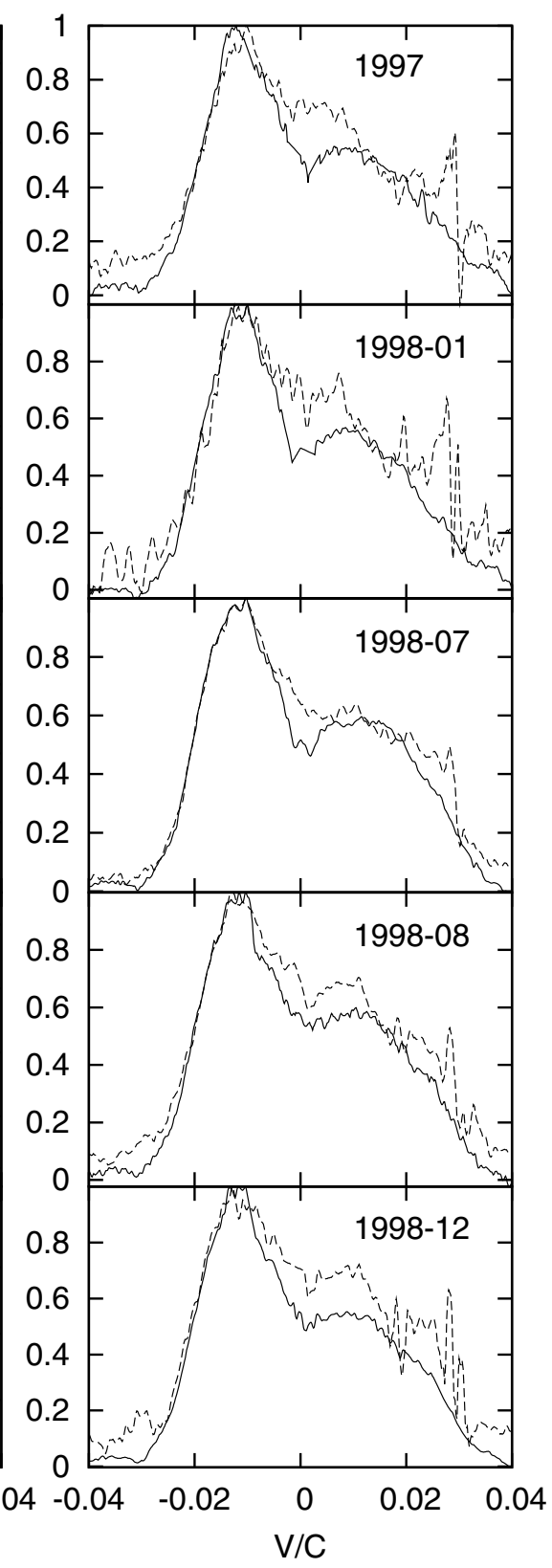

Fig. 11. The comparison of the $\mathrm{H} \alpha$ (solid line) and $\mathrm{H} \beta$ (dashed line) intensity (left) and their normalized profiles (right). flux variability $F$ (var) $=0.46$ in $1995-2007$ was the same as that of the $F$ (var) UV-continuum at wavelength $1370 \AA$ observed in 1994-1996.

\subsection{Correlations and cross-correlations(CCF) analysis}

To estimate the size and structure of the BLR, one can derive the cross-correlation function $(\mathrm{CCF})$ of the continuum light curve with the emission-line light curves. There are several ways to construct a CCF, and it is always advisable to use two or more methods to confirm the obtained results. Therefore, we cross-correlated the $5100 \AA$ continuum light curve with both the $\mathrm{H} \beta$ and $\mathrm{H} \alpha$ line (and their parts) light curves using two methods: (i) the $z$-transformed discrete correlation function (ZDCF) method introduced by Alexander (1997), and (ii) the interpolation cross-correlation function method (ICCF) described by Bischof \& Kollatschny (1999).
We choose to apply the CCF method introduced by Alexander (1997), i.e., the $z$-transformed discrete correlation function (ZDCF). The ZDCF approximates the bin distribution using a bi-normal distribution (see also Shapovalova et al. 2008). In the ZDCF the binning is performed by ensuring that there are equal population, while the effect of measurement errors is estimated by performing the Monte Carlo simulations (usually 100 runs is sufficient) of the averaged ZDCF of light curves with simulated random errors. Since a priori models of light curves do not exist, the estimated ZDCF errors may be extremely large. The $Z$ transforms convergence requires a minimum of 11 points per bin. Using this method, we obtained a lag of $96_{-47}^{+28}$ with coefficient of correlation of $0.92 \pm 0.02$ between the continuum and $\mathrm{H} \beta$. The cross-correlation functions obtained by ZDCF method are shown in Fig. 17. The lags and CCF coefficients are given in Table 6. As can be seen for $\mathrm{H} \alpha$, the CCF is affected by noise because of the small amount of observational data, and it is difficult to reliably determine the lag, there being two peaks in 
A. I. Shapovalova et al.: Spectral monitoring of 3C 390.3. I.

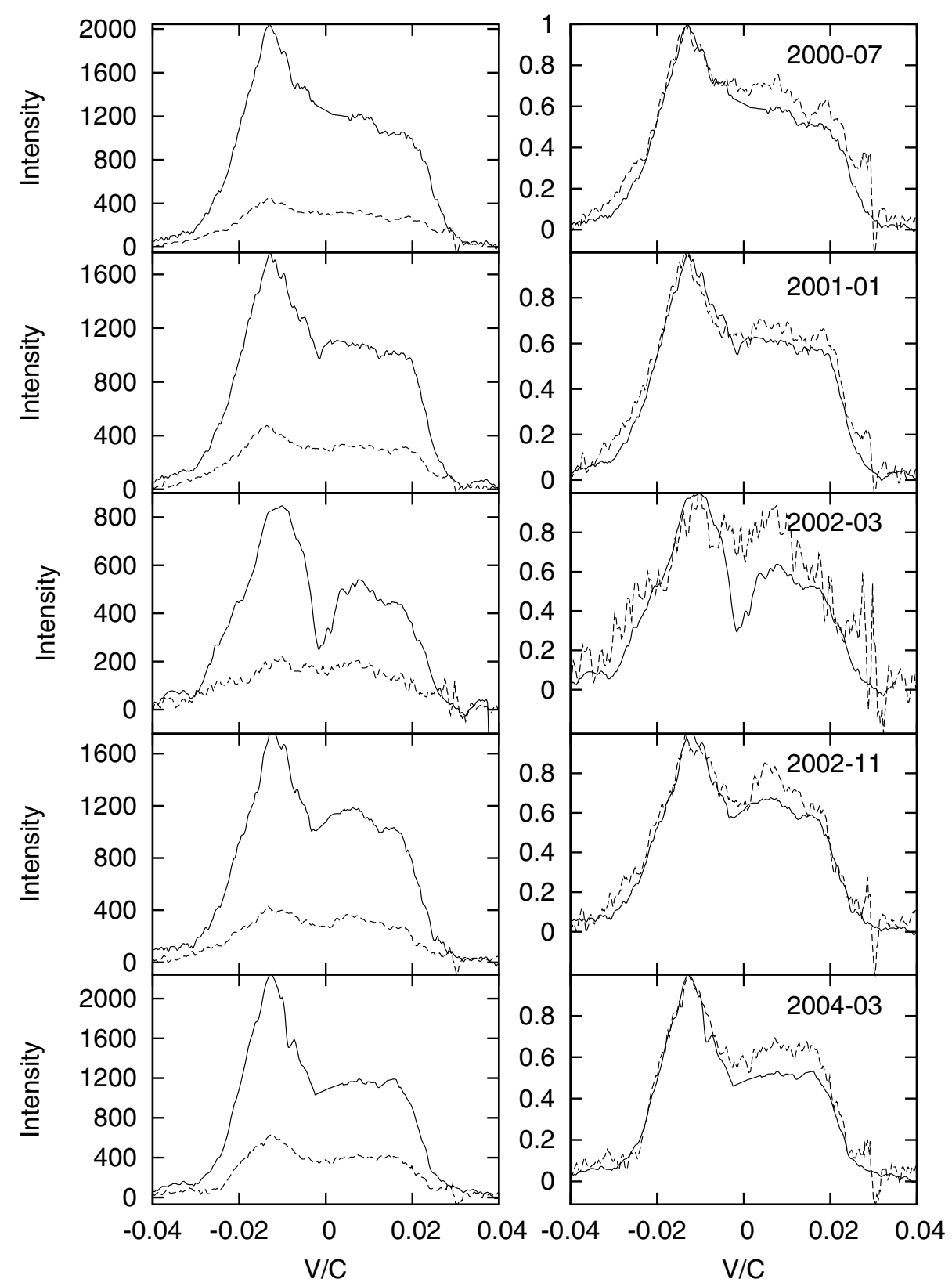

Fig. 11. continued.

Table 5. Variability parameters.

\begin{tabular}{lccccc}
\hline \hline Feature & Region (obs) $[\AA]$ & $F(\text { mean })^{a}$ & $\sigma(F)^{a}$ & $R(\max ) / R(\min )$ & $F($ var $)$ \\
\hline continuum & $5369-5399$ & 2.31 & 1.07 & 5.2 & 0.46 \\
$\mathrm{H} \beta$-all & $4909-5353$ & 2.39 & 0.91 & 4.7 & 0.38 \\
$\mathrm{H} \alpha$-all & $6740-7160$ & 9.42 & 3.36 & 3.4 & 0.35 \\
$\mathrm{H} \beta$-blue wing & $4977-5097$ & 0.88 & 0.37 & 4.9 & 0.41 \\
$\mathrm{H} \beta$-core & $5097-5165$ & 0.63 & 0.24 & 4.7 & 0.38 \\
$\mathrm{H} \beta$-red wing & $5165-5268$ & 0.70 & 0.26 & 4.3 & 0.37 \\
$\mathrm{H} \alpha$-blue wing & $6720-6882$ & 4.13 & 1.70 & 3.5 & 0.40 \\
$\mathrm{H} \alpha$-core & $6882-6974$ & 2.63 & 1.16 & 4.3 & 0.42 \\
$\mathrm{H} \alpha$-red wing & $6974-7136$ & 2.97 & 1.07 & 3.4 & 0.35 \\
$\mathrm{H} \beta$-blue part & $4977-5131$ & 1.20 & 0.48 & 4.8 & 0.39 \\
$\mathrm{H} \beta$-red part & $5131-5268$ & 1.00 & 0.38 & 4.5 & 0.38 \\
$\mathrm{H} \alpha$-blue part & $6720-6928$ & 5.46 & 2.23 & 3.6 & 0.40 \\
$\mathrm{H} \alpha$-red part & $6928-7136$ & 4.24 & 1.66 & 3.7 & 0.38 \\
\hline
\end{tabular}

Notes. Column (1): the mean flux. Column (2): the flux standard deviation. Column (3): the ratio of the maximum to the minimum flux in the monitoring period. Column (4): the measured (uncertainty-corrected) estimate of the variation amplitude with respect to the mean flux.

(a) The continuum flux is in units $10^{-15} \mathrm{erg} \mathrm{cm}^{-2} \mathrm{~s}^{-1} \mathrm{~A}^{-1}$, and the line flux is in $10^{-13} \mathrm{erg} \mathrm{cm}^{-2} \mathrm{~s}^{-1}$. 

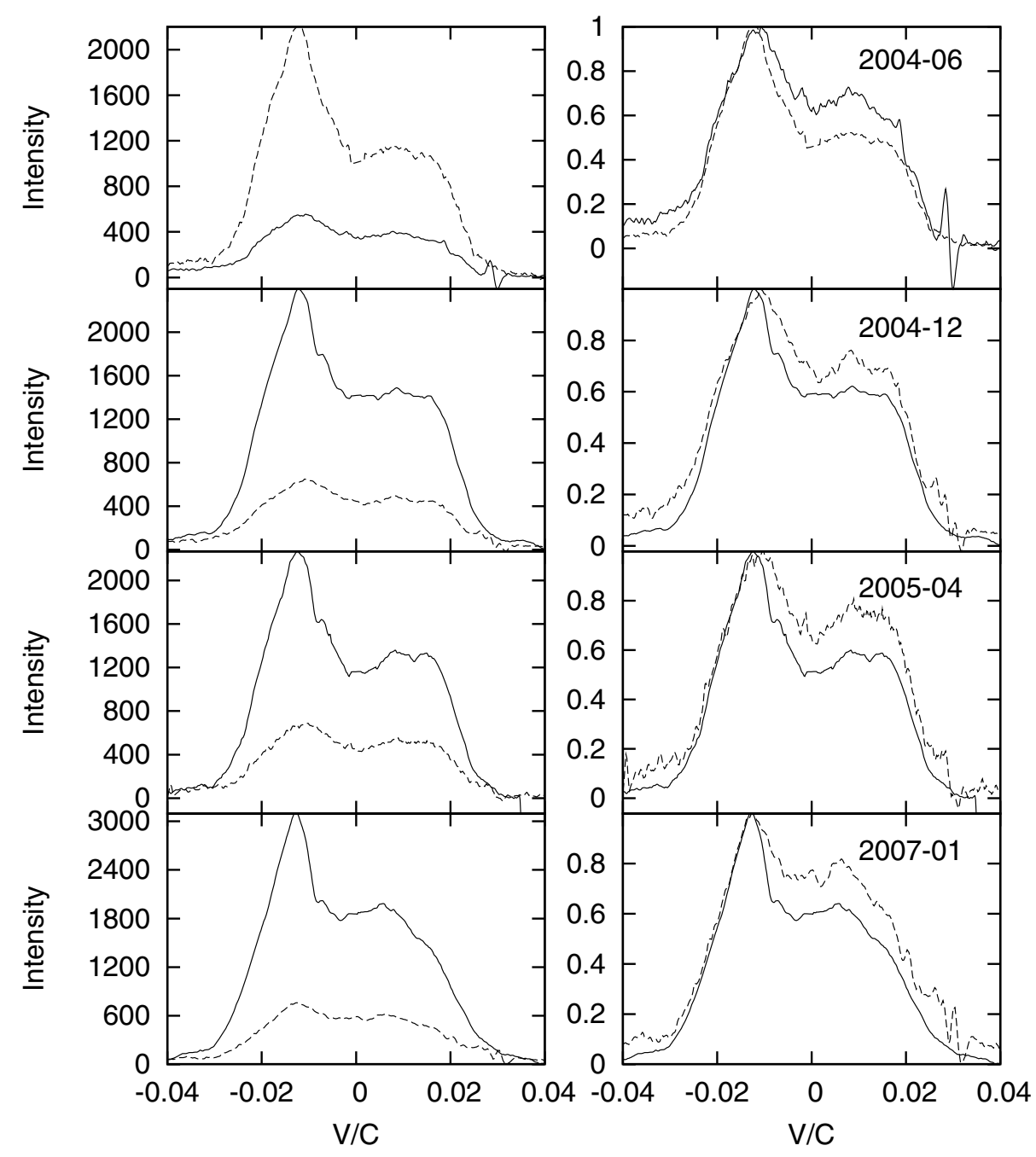

Fig. 11. continued.
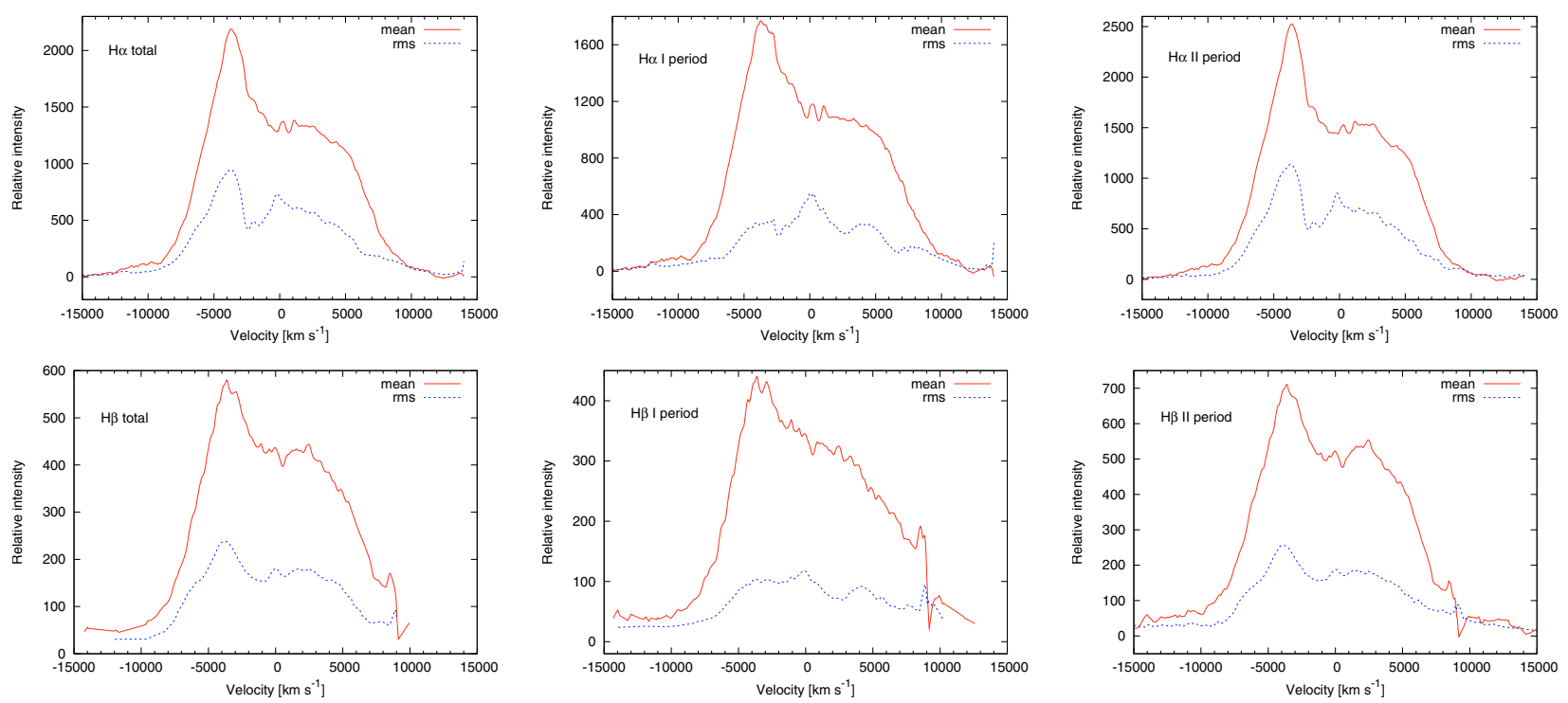

Fig. 12. Mean and rms spectra of the $\mathrm{H} \alpha$ (top) and $\mathrm{H} \beta$ (down) from the whole monitoring period (two left panels) and in the period I (1995-2002, two middle panels) and period II (2002-2007, two right panels).

the CCF curve with estimated lags of 24 days $\left(0.90_{0.05}^{+0.07}\right)$ and 151 days $\left(0.90_{-0.05}^{+0.06}\right)$.

As we mentioned above, we applied in addition the ICCF method to cross-correlate the flux of the continuum with both the
$\mathrm{H} \beta$ and $\mathrm{H} \alpha$ fluxes, and found lags of $97 \pm 17$ days $(\mathrm{CCF}=0.95)$ for $\mathrm{H} \beta$ and $270 \pm 150$ days $(\mathrm{CCF}=0.84)$ for $\mathrm{H} \alpha$, which agree with the ZDCF analysis. Both methods indicate that the lag between the continuum and $\mathrm{H} \beta$ flux is around 95 days. For the $\mathrm{H} \alpha$ line, the ICCF method is not applicable (because of the 

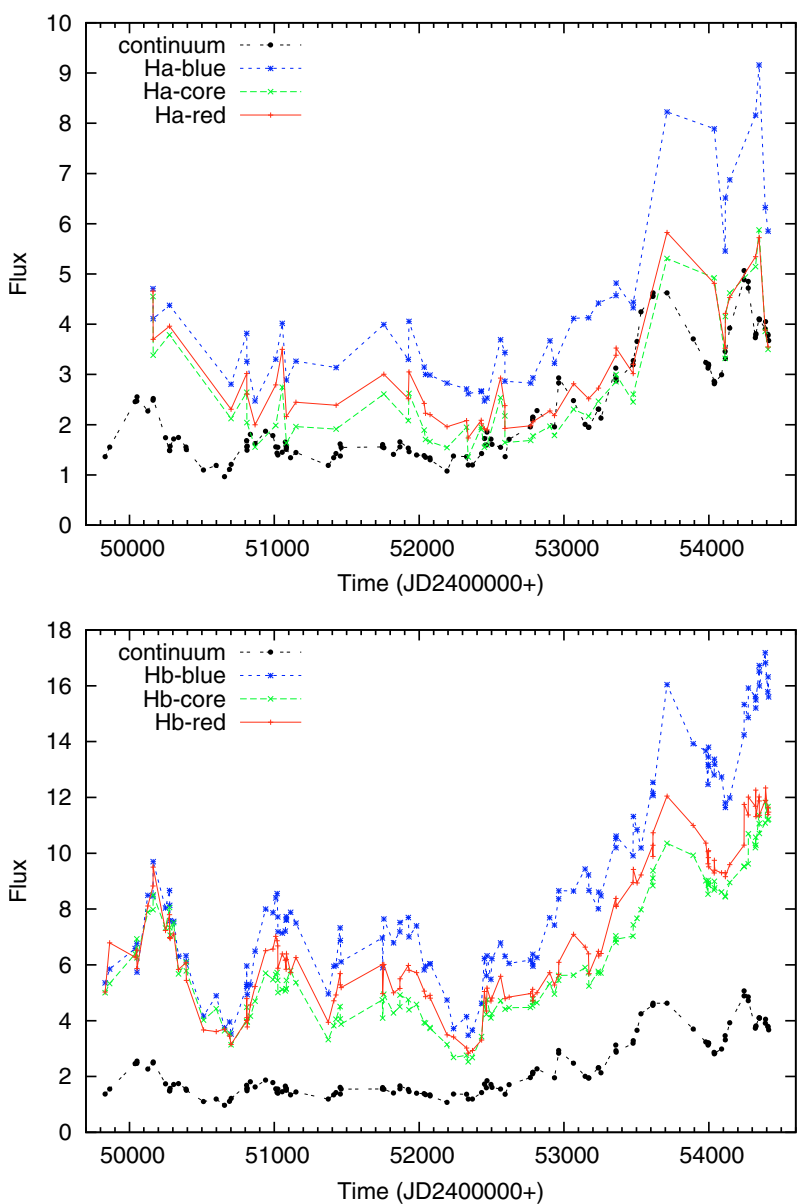

Fig. 13. JD-fluxes in the blue and red wings, and core and for $\mathrm{H} \alpha$ (upper panel) and $\mathrm{H} \beta$ (bottom panel). The continuum flux is given in $10^{-15} \mathrm{erg} \mathrm{cm}^{-2} \mathrm{~s}^{-1} \AA^{-1}, \mathrm{H} \beta$ in $10^{-14} \mathrm{erg} \mathrm{cm}^{-2} \mathrm{~s}^{-1}$ and $\mathrm{H} \alpha$ line in $10^{-13} \mathrm{erg} \mathrm{cm}^{-2} \mathrm{~s}^{-1}$.

small amount of observations). To confirm the results obtained we compiled centroids for $\mathrm{H} \alpha$ (see Table 6) and measured a lag of $127 \pm 18$ days, which is closer to the larger value obtained from ZDCF.

We also correlated the continuum and $\mathrm{H} \beta$ functions $F_{\mathrm{A}}(t)$, $F_{\mathrm{B}}(t)$, and $G(t)$ described in Sect. 3.2 and found no lag between the continuum-H $\beta F_{\mathrm{A}}(t)$, while the lag between the continuum$\mathrm{H} \beta F_{\mathrm{B}}(t)$ is $574_{-26}^{+12}$ days $\left(0.74_{-0.06}^{+0.07}\right)$. It is interesting that the lag between $G^{\mathrm{c}}(t)$ and $G^{\mathrm{l}}(t)$ is close to the lag of the entire line within the errorbars and is $97_{-46}^{+27}\left(0.59_{-0.07}^{+0.08}\right)$. The CCFs between these three function are presented in Fig. 18.

The calculated lags and CCF between the continuum and different parts of lines, as well as between different parts of $\mathrm{H} \alpha$ and $\mathrm{H} \beta$ using ZDCF method are given in Table 6. The last two columns of Table 6 indicate the centroid lag and the $90 \%$ of the ZDCF maximum, respectively.

In addition, we searched for any linearity between either the $\mathrm{H} \beta$ and continuum flux, or the $\mathrm{H} \alpha$ and continuum flux, taking different time shifts for the continuum. We assumed that the continuum is shifted by $0,30,100,200$, and 300 days. As shown Figs. 19 and 20, the best-fit linear correlation $\mathrm{H} \alpha$ versus (vs.) continuum flux is found when the continuum has a lag of 200 days, but for $\mathrm{H} \beta$ the clearest linearity is obtained for a continuum shift of 100 days. This agrees well with the results obtained for the CCF analysis mentioned above.
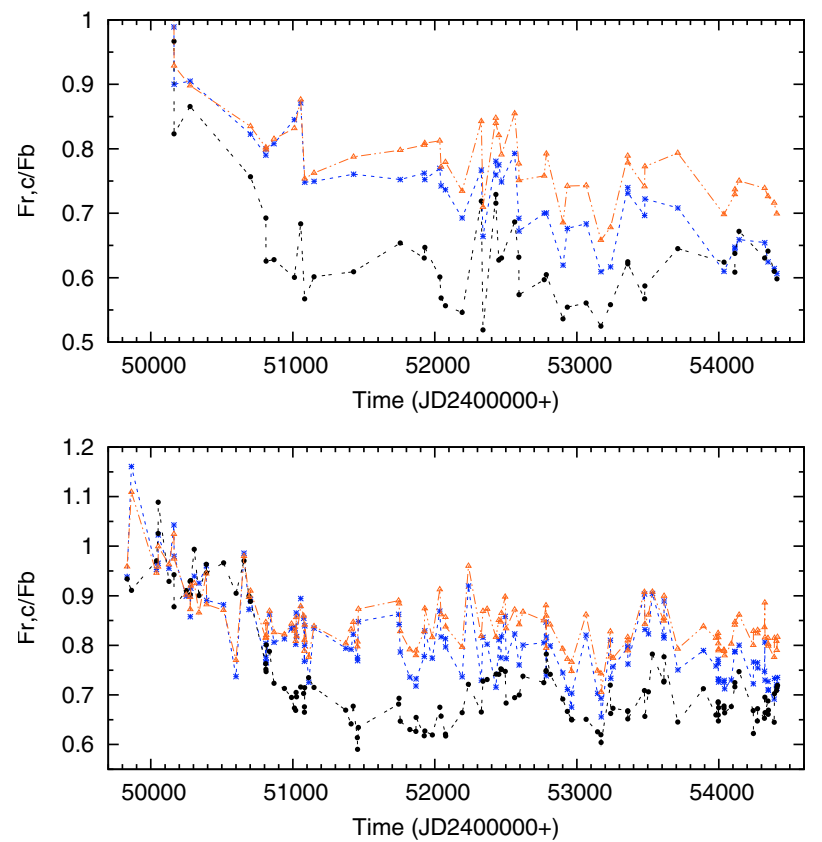

Fig. 14. The variation in the ratio red/blue (asterisk blue-line) and central/blue (circles black-line) of the $\mathrm{H} \alpha$ (up) and $\mathrm{H} \beta$ (bottom) line component fluxes. The triangle red-line represents the red/blue flux ratio for when we divided lines into only two parts (blue and red).

\subsubsection{The lines, line-parts, and continuum correlations}

We correlated the continuum and line parts, as well as the line parts with the other line parts, finding that the lag in the continuum vs. $\mathrm{H} \beta$ line parts is $96_{-28}^{+47}$ in all cases (the CCF coefficient being larger than 0.9). This and a short lag in the $\mathrm{H} \beta$ line part line part lag $\left(6_{-6}^{+36}\right.$, practically around zero within the errorbars) indicate that total line responses to the continuum vary at the same time. In both cases, there is no significant lag between the blue and red $\mathrm{H} \beta$ line parts, when we consider only blue and red part, and in the second case when we consider the blue, central, and red part.

For $\mathrm{H} \alpha$, Table 6 shows that may be same lag between the blue and red/central part, around 23 days. However, since for $\mathrm{H} \alpha$ there are fewer data available for the calculation than for $\mathrm{H} \beta$, as well as that there is one peak around zero (with a slightly smaller CCF value; see values in brackets in Table 6), this should be interpreted with care.

The CCFs for both $\mathrm{H} \alpha$ and $\mathrm{H} \beta$ in addition to the CCFs between their parts are shown in Fig. 17. On the left side of Fig. 17 (from top to bottom), we present the $\mathrm{H} \beta \mathrm{CCFs}$ of the continuumtotal line flux, blue-core, blue-red (three parts), continuum-red, and continuum-blue. On the right side, we present the same for $\mathrm{H} \alpha$ line. The CCFs for $\mathrm{H} \alpha$ are clearly noisier than for $\mathrm{H} \beta$ because there are fewer observations of $\mathrm{H} \alpha$ than $\mathrm{H} \beta$.

\section{Discussion of results}

We have presented and analyzed data of the long-term monitoring of 3C 390.3 (13 years, from 1995 to 2007). In Paper I, we presented the monitoring results for the period 1995-1999, and here we have enlarged our data set for the 8-year period 1999-2007. We now discuss the derived results. 

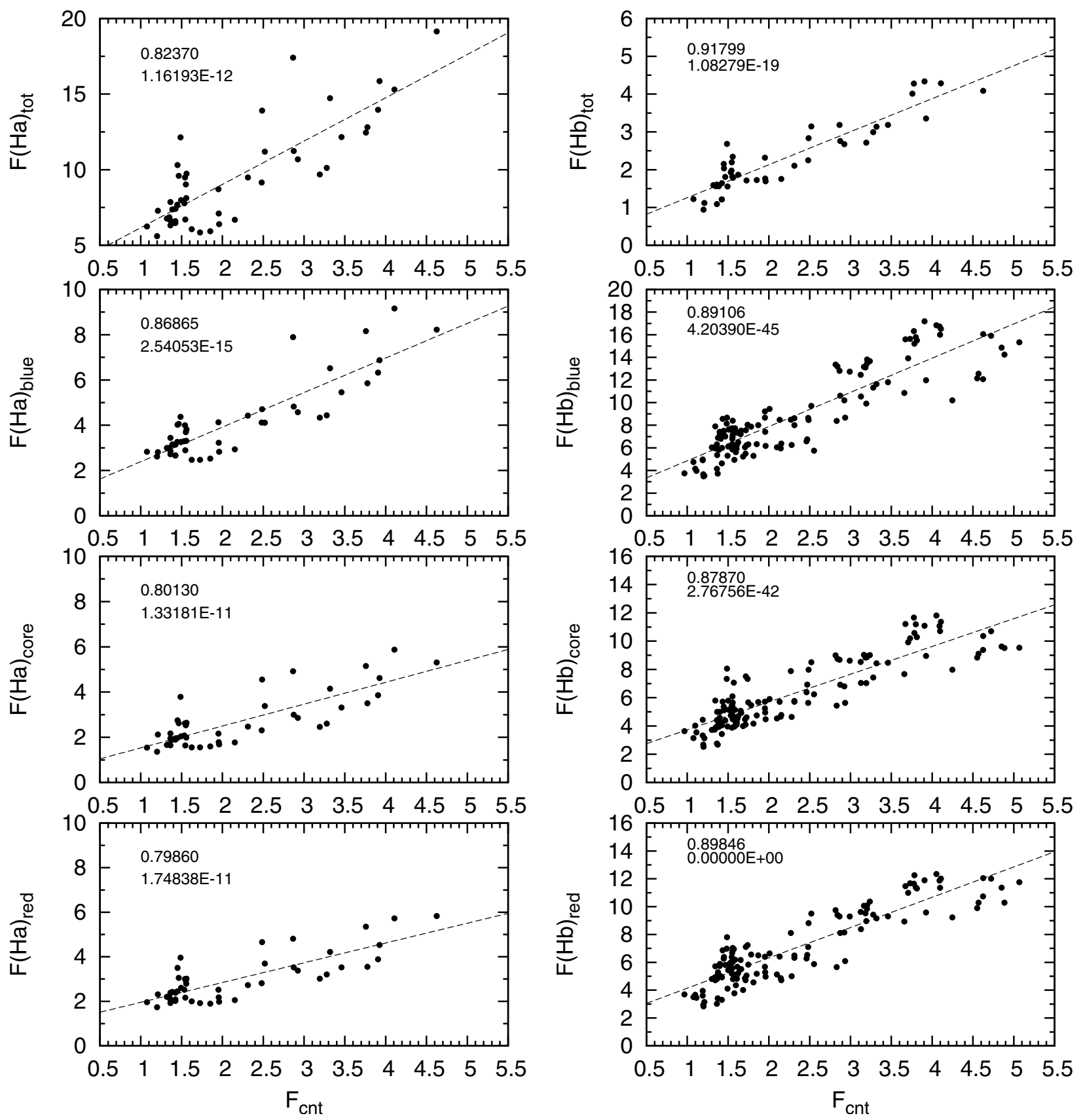

Fig. 15. The relationships between the $\mathrm{H} \alpha$ (left) and $\mathrm{H} \beta$ (right) total line and line parts with the continuum flux. The continuum flux is given in $10^{-15} \mathrm{erg} \mathrm{cm}^{-2} \mathrm{~s}^{-1} \AA^{-1}, \mathrm{H} \beta$ in $10^{-14} \mathrm{erg} \mathrm{cm}^{-2} \mathrm{~s}^{-1}$ and $\mathrm{H} \alpha$ line in $10^{-13} \mathrm{erg} \mathrm{cm}^{-2} \mathrm{~s}^{-1}$. The correlation coefficient and the $p$-value are given in the upper left corner.

\subsection{Continuum and line light curves: possible quasi-periodical oscillation}

In the first period (1995-2002), three maxima (outbursts) are discernible in the $\mathrm{H} \beta$ and continuum light curves, after which in 2002 , the activity seems to reach a minimum. Besides these three maxima, there are several local peaks, even in the minimum state, which became enhanced during the maximum of activity (2003-2007). This motivated us to search periodicity in the light curves of both $\mathrm{H} \beta$ and continuum (we did not attempt this for $\mathrm{H} \alpha$ because there were fewer data than for $\mathrm{H} \beta$ ). By applying the two methods, we showed that there is a high probability that in both the line and continuum light curves some quasi-periodical oscillations exist, i.e., there is some long-term periodicity over between 1.65 and 4 years. Tao et al. (2008) constructed a historical light curve from 1894 to 2004 and identified possible periods of $8.30,5.37,3.51$, and $2.13 \mathrm{yr}$.

The appearance of quasi-periodic oscillation (QPOs) is often related to accretion flows around black holes. Chakrabarti et al. (2004) performed numerical simulations of two-dimensional axisymmetric accretion flows around both stellar and supermassive black holes and found that due to both the radial and vertical oscillation of shock waves in the accretion flow, the quasi-periodic variabilities in the light curve can be expected. These quasi-periodic variabilities may be detectable because of the predominance of the interaction between the winds and the inflow (see Chakrabarti et al. 2004, and references therein). Chakrabarti et al. (2004) found that the QPO frequency of supermassive black hole should be approximately $10^{-6}-10^{-7} \mathrm{~Hz}$, 

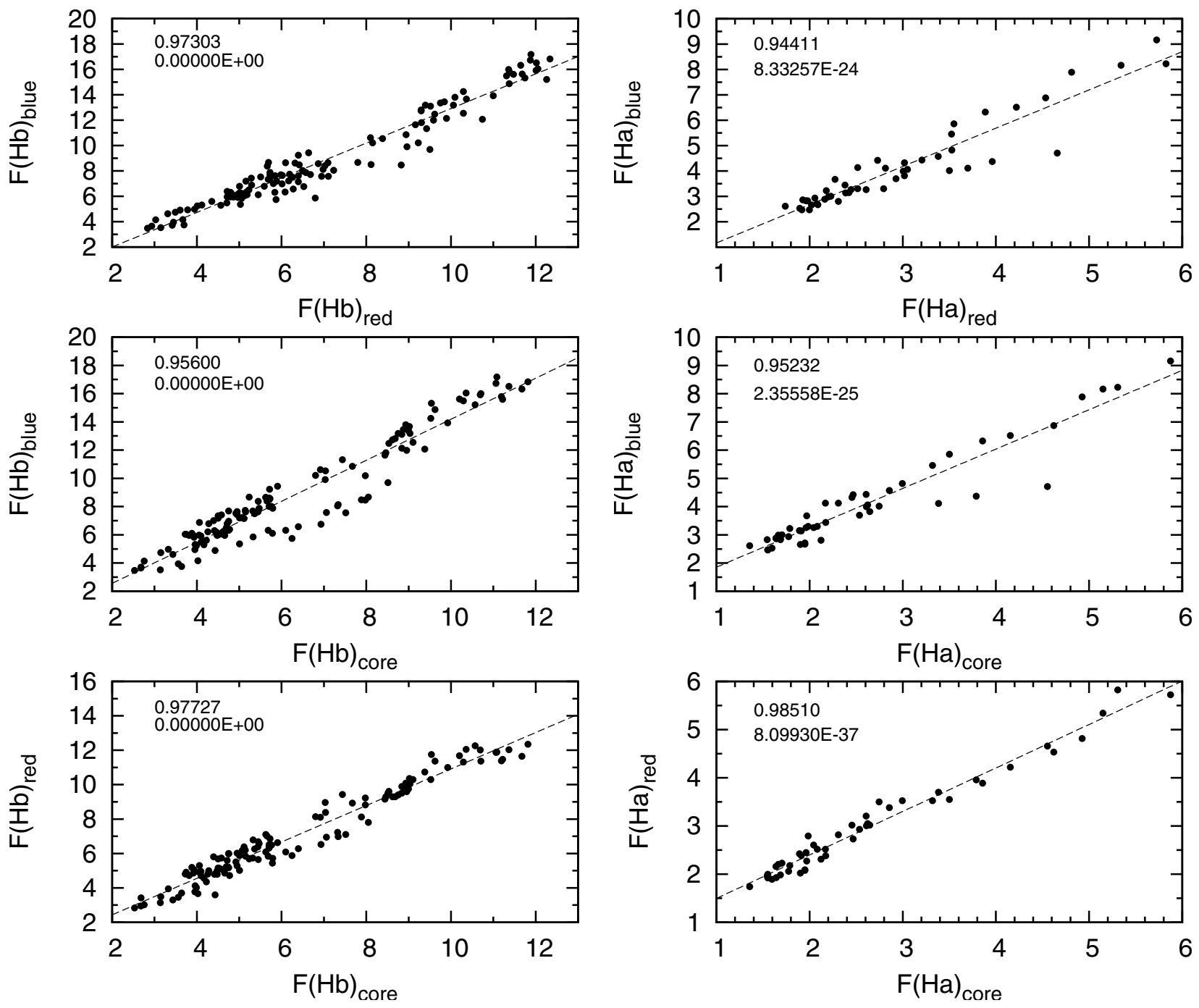

Fig. 16. Relationships between the $\mathrm{H} \beta$ (left) and $\mathrm{H} \alpha$ (right) line segments. The $\mathrm{H} \beta$ flux is given in units $10^{-14} \mathrm{erg} \mathrm{m}^{-2} \mathrm{~s}^{-1}$ and $\mathrm{H} \alpha$ in $10^{-13} \mathrm{erg} \mathrm{m}^{-2} \mathrm{~s}^{-1}$. The correlation coefficient and the $p$-value are given in the upper left corner.

and the frequencies of possible oscillations were found to be $\sim 10^{-8}$. These variabilities are consistent with a disk geometry. Gierliński et al. (2008) reported that QPO is detected in X-ray spectra of RE J1034+396 (a narrow-line Seyfert 1 galaxy), but the QPO does not appear to have a disk origin (see Middleton et al. 2009).

Harris et al. (2009) also reported the discovery of a X-ray QPO in the M 87 jet. Arshakian et al. (2010) found a connection between the variable optical continuum and the subparsecscale jet in 3C 390.3, in addition to a significant correlation (at a confidence level of $>99.99 \%$ ) between the ejected jet components and optical continuum flares and that radio events follow the maxima of optical flares with the mean time delay around $\sim 36$ days. Results obtained in León-Tavares et al. (2010) for the broad-line radio galaxy 3C 120 , confirm the correlations found in Arshakian et al. (2010) and support the idea that the correlation between optical flares and kinematics of the jet may be common to all radio-loud galaxies and quasars. There is therefore, an open question about the origin of QPOs in 3C 390.3.

We note that quasi-periodical variations in the observed blueto-red line wing flux ratios of $\mathrm{H} \beta$, of period about 10 years, have been reported in several papers (see Veilleux \& Zheng 1991; Shapovalova et al. 1996; Bochkarev et al. 1997), maximum values occurring at 1975, 1985, and 1995. From our data (Sect. 3.4, Fig. 14), it follows that the maximal ratio $F$ (blue) $/ F$ (red) was observed in 2004-2006. In Figs. 6, 10b, 13, the fluxes of maximal amplitude clearly occur in the years 1996 and 2006, e.g. they divide the interval by about 10 years. This closely agrees with reported quasi-periodical variations of period $\sim 10$ years. This period of 10 years may be connected with the BLR (disk) rotation period.

\subsection{Dimension of the BLR: CCF analysis}

In Table 6 , the correlations between the continuum flux and either $\mathrm{H} \alpha$ or $\mathrm{H} \beta$ give different values, indicating different sizes of the $\mathrm{H} \alpha$ and $\mathrm{H} \beta$ emission regions. The lag measured for $\mathrm{H} \beta$ is $~ 95$ days, while for $\mathrm{H} \alpha$ it is closer to 100 days. In addition, the coefficient of variability (see Table 5 ) is slightly higher for $\mathrm{H} \beta$ than $\mathrm{H} \alpha$, implying that the $\mathrm{H} \beta$ emitting region may be more compact than that of $\mathrm{H} \alpha$.

In Paper I, the time lag in the response of the emission line relative to flux variations in the continuum, has been found to vary with time i.e. during 1995-1997 a lag of about 100 days was detected, while during 1998-1999, a double-valued lag of $\approx 100$ days and $\approx 35$ days is present. It is interesting that we obtained a similar result from $\mathrm{H} \alpha$, while for $\mathrm{H} \beta$ we obtained 


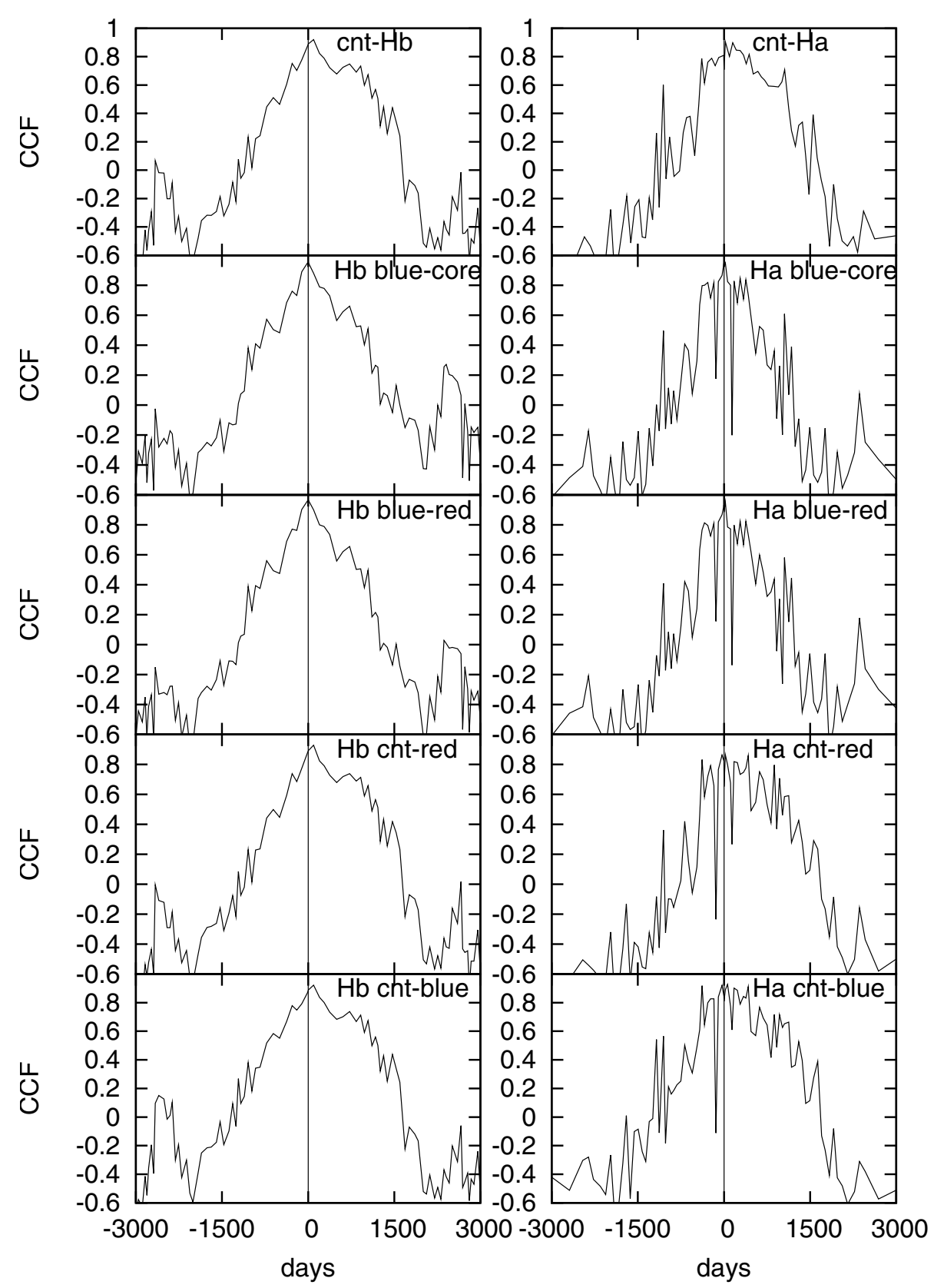

Fig. 17. CCFs for $\mathrm{H} \beta$ (left) and $\mathrm{H} \alpha(r i g h t)$.

consistent lag of 95 days using both methods. This, and also difference in the FWHM may indicate multicomponent origin of broad lines, as e.g. in the classical accretion disk, and one disklike region formed around the accretion disk.

We recall the BLR scenario proposed by Arshakian et al. (2010), where the broad emission lines of a double-peaked structure originate in two kinematically and physically different regions of 3C 390.3: 1) BLR1 - a traditional BLR (accretion disk (AD) and the surrounding gas), which is at the distance $\sim 30$ light days from the nuclei; 2) BLR2 - a subrelativistic outflow surrounding the jet that is in the cone that is within $\sim 100$ light days of the source, at a distance of $\sim 0.4 \mathrm{pc}$ from the central engine. During the maximal-brightness periods of the nucleus, most of the continuum variable radiation is emitted from the jet and ionizes the surrounding gas, creating the BLR2 that then produces the broad line emission. During the brightness minima, the jet contribution to the ionizing continuum diminishes and the main broad-line emission originates in the "classical" BLR1 (AD), ionized by nuclei continuum related to accretion onto the $\mathrm{BH}$.
One can speculate that this scenario can be used to explain different lags of $\mathrm{H} \alpha$ and $\mathrm{H} \beta$ (and difference in the rate of variation), but there may also be some perturbation in a "classical" accretion disk in combination with the different dimensions of the emitting regions that may explain the observed results. In a forthcoming paper, we propose to apply disk models to explore these findings.

\subsection{The structure of the BLR - disk and perturbations}

An accretion disk is usually assumed to produce the doublepeaked line profiles emitted by some AGN. Gezari et al. (2007) found, from their approximately 20-year long spectral monitoring of the double-peaked $\mathrm{H} \alpha$ emission line of seven broad-line radio galaxies, that their profiles varied on timescales of from months to years, and double-peaked lines were successfully modeled by emission of the gas from the outer part of the accretion disk. To explain variable flux ratios of the line wings, more 
A. I. Shapovalova et al.: Spectral monitoring of 3C 390.3. I.

Table 6. The time lag and CCF coefficient for the continuum and $\mathrm{H} \beta, \alpha$ line (total line and parts) using ZDCF method.

\begin{tabular}{|c|c|c|c|c|c|}
\hline Pair I & Pair II & $\begin{array}{c}\text { Lag }(\text { ZDCF }) \\
\text { (days) }\end{array}$ & $\mathrm{ZDCF}$ & $\begin{array}{c}\text { Lag (centroid) } \\
\text { (days) }\end{array}$ & $90 \% \operatorname{Max}_{\mathrm{ZDCF}}$ \\
\hline$F_{\mathrm{cnt}}$ & $F(\mathrm{H} \beta)_{\mathrm{tot}}$ & $96_{-47}^{+28}$ & $0.92_{-0.02}^{+0.02}$ & $93_{-18}^{+20}$ & 0.83 \\
\hline$F_{\mathrm{cnt}}$ & $F(\mathrm{H} \beta)_{\text {blue }}$ & $96_{-28}^{+47}$ & $0.92_{-0.02}^{+0.02}$ & $71_{-26}^{+34}$ & 0.83 \\
\hline$F_{\mathrm{cnt}}$ & $F(\mathrm{H} \beta)_{\mathrm{red}}$ & $96_{-28}^{+47}$ & $0.92_{-0.02}^{-0.02}$ & $70_{-26}^{+36}$ & 0.83 \\
\hline$F_{\text {cnt }}$ & $F(\mathrm{H} \beta)_{\text {core }}$ & $96_{-28}^{+47}$ & $0.91_{-0.02}^{+0.02}$ & $72_{-26}^{-30}$ & 0.82 \\
\hline $\mathrm{F}(\mathrm{H} \beta)_{\text {blue }}$ & $F(\mathrm{H} \beta)_{\text {red }}$ & $6_{-6}^{+36}$ & $0.96_{-0.01}^{-0.02}$ & $-5_{-30}^{+28}$ & 0.87 \\
\hline $\mathrm{F}(\mathrm{H} \beta)_{\text {blue }}$ & $F(\mathrm{H} \beta)_{\text {core }}$ & $6_{-6}^{+36}$ & $0.94_{-0.01}^{+0.01}$ & $-4_{-29}^{+28}$ & 0.85 \\
\hline \multirow[t]{2}{*}{$F_{\mathrm{cnt}}$} & $F(\mathrm{H} \alpha)_{\mathrm{tot}}$ & $24_{-5}^{+7}$ & $0.90_{-0.07}^{+0.05}$ & $127_{-18}^{+18}$ & 0.81 \\
\hline & & $\left(151_{-9}^{+20}\right)$ & $0.90\left({ }_{-0.06}^{+0.05}\right)$ & & \\
\hline$F_{\mathrm{cnt}}$ & $F(\mathrm{H} \alpha)_{\text {blue }}$ & $\begin{array}{c}64_{-21}^{+26} \\
\left(175_{-12}^{+25}\right)\end{array}$ & $\begin{array}{c}0.92_{-0.06}^{+0.04} \\
\left(0.90_{-0.06}^{+0.05}\right)\end{array}$ & $76_{-13}^{+14}$ & 0.83 \\
\hline$F_{\mathrm{cnt}}$ & $F(\mathrm{H} \alpha)_{\mathrm{red}}$ & $\begin{array}{c}23_{-5}^{+7} \\
\left(175_{-12}^{+24}\right)\end{array}$ & $\begin{array}{c}0.87_{-0.09}^{+0.07} \\
\left(0.81_{-0.10}^{+0.08}\right)\end{array}$ & $75_{-14}^{+14}$ & 0.79 \\
\hline$F_{\mathrm{cnt}}$ & $F(\mathrm{H} \alpha)_{\text {core }}$ & $\begin{array}{c}64_{-21}^{+26} \\
\left(175_{-12}^{+25}\right)\end{array}$ & $\begin{array}{c}0.90_{-0.07}^{+0.06} \\
\left(0.81_{-0.08}^{+0.08}\right)\end{array}$ & $76_{-13}^{+14}$ & 0.81 \\
\hline $\mathrm{F}(\mathrm{H} \alpha)_{\text {blue }}$ & $F(\mathrm{H} \alpha)_{\mathrm{red}}$ & $\begin{array}{c}23_{-5}^{+7} \\
\left(-1_{-8}^{+1}\right)\end{array}$ & $\begin{array}{c}0.95_{-0.04}^{+0.03} \\
\left(0.93_{-0.03}^{+0.02}\right)\end{array}$ & $10_{-9}^{+8}$ & 0.85 \\
\hline $\mathrm{F}(\mathrm{H} \alpha)_{\text {blue }}$ & $F(\mathrm{H} \alpha)_{\text {core }}$ & $\begin{array}{c}23_{-5}^{+7} \\
\left(-1_{-8}^{+1}\right)\end{array}$ & $\begin{array}{c}0.95_{-0.03}^{+0.02} \\
\left(0.93_{-0.03}^{+0.02}\right)\end{array}$ & $50_{-11}^{+13}$ & 0.86 \\
\hline
\end{tabular}

Notes. For a ZDCF performed between the continuum and $\mathrm{H} \alpha$, two peaks (within the ZDCF errorbars) are very often present, and the value for the second peak is given in brackets. The last two columns give the centroid lag and the $90 \%$ of the ZDCF maximum.

complex models are required, such as hot spots (Zheng 1996), two-arm spiral waves in the accretion disk (Chakrabarti \& Wiita 1994), or a relativistic eccentric disk (Eracleous et al. 1995). Therefore, accretion-disk emission from 3C 390.3 is probably present, and investigation of the long-term variability in the spectra (broad lines and continuum) of this object can provide information about the accretion disk structure.

As mentioned in Paper I (and papers quoted in Sect. 1), the geometry of the BLR of 3C 390.3 probably has a disk-like structure.

From Table 4, we conclude that the distance (in velocity) between the blue and red peaks was smaller in 2002-2007, when the continuum flux was relatively high, while it was larger when the continuum flux was relatively weak. The similar anticorrelation in 1995-1999 was found in Paper I. This can be expected in the case where broad emission lines originate in the $\mathrm{AD}$, i.e., are produced by changes in the dimension of the disk. The velocity difference increases, corresponding to smaller radii (emission from the part of the disk near to the $\mathrm{BH}$ ), as the continuum flux decreases. When the continuum flux increases the velocity difference decreases, i.e., the contribution of disk parts farther from the $\mathrm{BH}$ is more significant.

If the BLR, however, consists of 2 regions - AD and subparcsec-jet (Arshakian et al. 2010), then, in the maximum activity state, most radiation is produced by the jet and the velocity difference between blue and red peak decreases (further from $\mathrm{BH}$ ). Otherwise, in contrast (i.e., in the minimum state of activity) most radiation is caused by AD and the velocity difference increases (near BH). We also note that in blazars, where the emission is dominated by the extremely variable, non-thermal radiation from the jet, the few spectroscopic studies available (since lines are often absent for these objects) have shown that in some cases there is a weak (or no) correlation between the line and continuum emission. This result was also reported by Benitez et al. (2009) on the basis of their spectroscopical monitoring of blazar 3C 454.3 from September 2003 to July 2008. They found that $\mathrm{Mg}$ II emission lines respond proportionally to the continuum variations when the source is in a low-activity state. In contrast, close to the optical outbursts detected in 2005 and 2007, the Mg II emission lines showed little response to the continuum flux variations.

In any case, the profile evolution indicates that some additional emission is present, as seen in the first period 1997-1998 for the central part of the line, and at the end of the monitoring campaign, when a difference was measured for the red wings of $\mathrm{H} \beta$ and $\mathrm{H} \alpha$ lines. These results could be interpreted by some kind of two-component model (see also Popović et al. 2004; Bon et al. 2009) but may also caused by the perturbations in the disk (due to shock waves), providing support for a disk model (as noted in Paper I). A pure disk model would be consistent with the absence of a lag between flux variations in the red and blue wings of the lines, and the line shapes of $\mathrm{H} \alpha$ and $\mathrm{H} \beta$ (with the blue and red peaks) being indicative of the relativistic disk emission.

If one were to propose pure disk geometry (without additional emission), then the various bumps in line profiles may be caused by spiral shocks in the disk. As we have shown in Sect. 3.2, the $\mathrm{H} \beta$ and continuum light curves can be decomposed into three functions $F_{\mathrm{A}}(t), F_{\mathrm{B}}(t)$, and $G(t)$. As discussed in Sect. 3.5, the time lag between $F_{\mathrm{A}}^{\mathrm{c}}(t)$ and $F_{\mathrm{A}}^{\mathrm{H} \beta}(t)$ is zero, while between $F_{\mathrm{B}}^{\mathrm{c}}(t)$ and $F_{\mathrm{B}}^{\mathrm{H} \beta}(t)$ it is around 600 days, which is close to the bump in CCF curves (see Fig. 17). The lag between the $G(t)$ functions corresponds to the lag between the continuum and line 

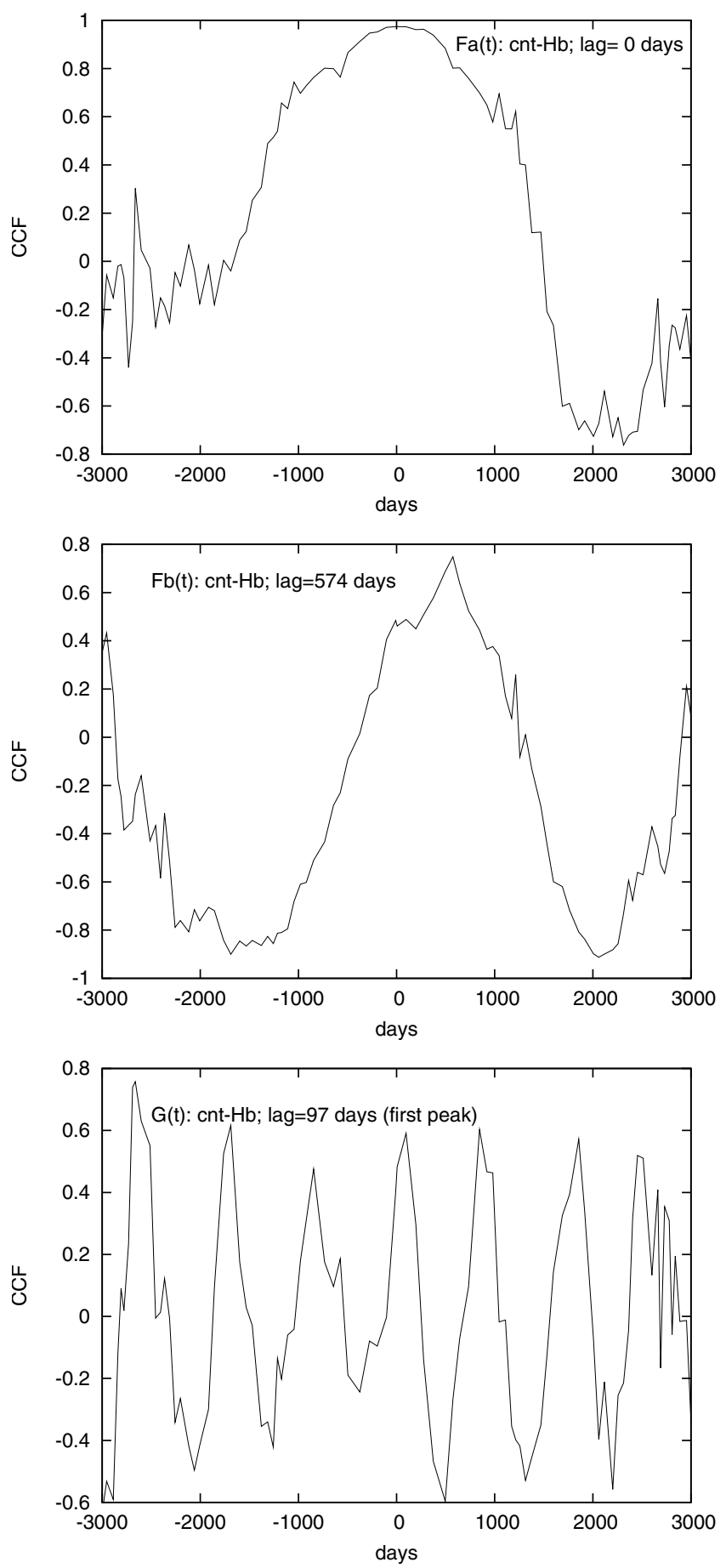

Fig. 18. CCFs between continuum and line functions $F_{\mathrm{A}}$ (top), $F_{\mathrm{B}}$ (middle) and $\mathrm{G}($ down).

fluxes. This may indicate that we have a complex scenario in which the variability has two distinct periods. Assuming emission to originate in the disk, if a perturbation appears close to the black hole, the continuum flux may first increase, after which (at around 90-100 days) the line flux increases. This is well described by the lag in the function $G(t)$. On the other side, one may expect some of the continuum to be emitted from the line emission region (as e.g. due to free-bound transitions), which may be described by $F_{\mathrm{A}}$ (lag zero between continuum and line). It is interesting that we have an additional lag of around 600 days in the flux $\left(F_{\mathrm{B}}\right)$, which may be caused by the perturbation seen in the flash, after which the perturbation spreads through the disk at super-sonic velocities.

The line profile variations and a possible model of the spiral shocks in the disk will be presented in Paper II of this series.

\subsection{Disk versus a bipolar radial outflow}

As noted in the Introduction, some models can explain the double-peaked broad emission line profiles in 3C 390.3. As aforementioned, a very popular model generates these lines in a relativistic disk. However, there is some contradiction between this model and the polarization observations of 3C 390.3. Smith et al. $(2004,2005)$ proposed a two-component scattering model to explain the polarization properties of the optical continuum and $\mathrm{H} \alpha$ broad emission lines in Sy1 nuclei. They hypothesized that both the compact equatorial scattering region located inside the torus and the polar scattering regions are present in all Seyfert galaxies, and that their polarization properties can be broadly understood in terms of an orientation effect. On the one hand, Smith et al. (2004) suggested that the broad emission lines originate in a rotating emission line disk surrounded by a coplanar scattering region. Thus, in polarization light a double-peaked profile of $\mathrm{H} \alpha$ will be observed, as in total light, and the degree of polarization will be maximal in the wings of the line and minimal in the line core. The position angle (PA) of polarization is aligned with the projected disk rotation axis and hence with the radio source axis (e.g. E-vector is parallel to the pc-jet axis). On the other hand, the polarization is caused by far-field scattering in the polar illumination cones of the circumnuclear torus (Smith et al. 2004). In polarization light, the $\mathrm{H} \alpha$ profile has only one central peak and the position angle (PA) of polarization is perpendicular to the projected disk rotation axis or pc-jet axis (e.g. E-vector is perpendicular to the pc-jet axis). From spectropolarimetric observations of $3 \mathrm{C} 390.3$ in $\mathrm{H} \alpha$ region taken at the $4.2 \mathrm{~m}$ Wiliam Herschel telescope in 1995 and 1997, Corbett et al. $(1998,2000)$ obtained the following main results:

a) the optical polarization $E$ vector is very closely aligned with the radio source axis, as for the case of equatorial scattering region;

b) in polarized light, the $\mathrm{H} \alpha$ profile has a broad central peak centered close to the systematic velocity and is also slightly asymmetric, the blue wing being somewhat less extended than the red. This result favors polar scattering and is inconsistent with an equatorial scattering model because a double peak in the polarized line profile is not observed;

c) The $\mathrm{P}(\lambda)$ - the percentage polarization of the continuum changed significantly between 1995 and 1997, presumably because there is a variable, intrinsically polarized, synchrotron contribution to the continuum (from a pc-jet component);

Corbett et al. (1998, 2000) considered a model in which $\mathrm{H} \alpha$ photons emitted by a biconical flow are scattered by the inner wall of the torus. Thus, the scattering plane is perpendicular to the radio jet (e.g. E-vector parallel to pc-jet axis) and produces a singlepeaked scattered $\mathrm{H} \alpha$ line profile. However, this model does not agree with the optical monitoring data, for which the following results are obtained:

1. the CCF-analysis for the $\mathrm{H} \beta$ and $\mathrm{H} \alpha$ line wings in $3 \mathrm{C} 390$ did not reveal any significant delay in the variations of the line wings with respect to the central part of the line, or relative to each other. The flux in the $\mathrm{H} \beta$ line wings and core also varied simultaneously. These results are indicative 
A. I. Shapovalova et al.: Spectral monitoring of 3C 390.3. I.
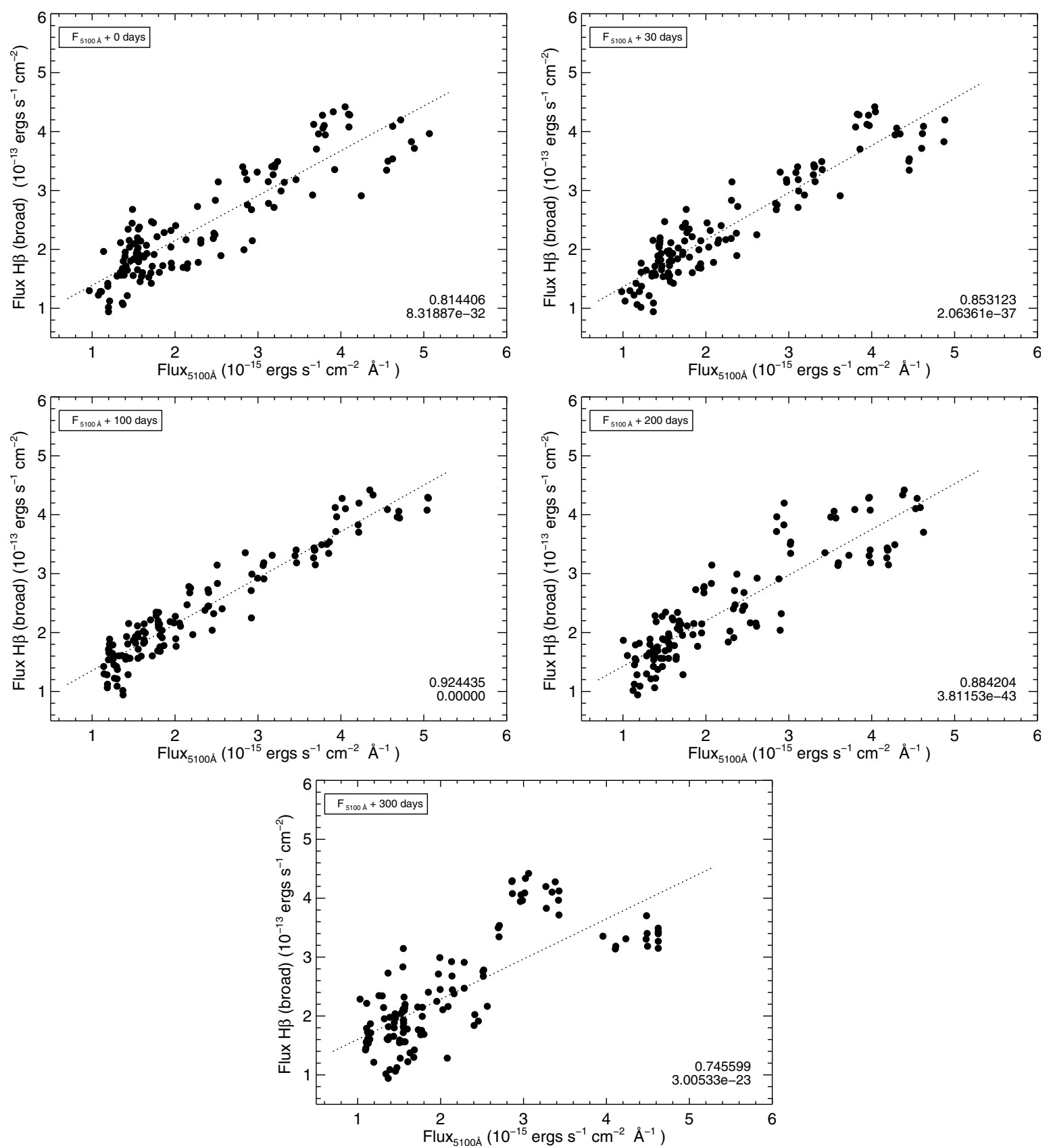

Fig. 19. $\mathrm{H} \beta$ vs. continuum correlations - with and without lags. Shifts for the continuum are given in the top left and the correlation coefficient and the $p$-value are in the bottom right.

of predominantly circular motions in the BLR and favor a model in which the main contribution to the broad lines flux originates in the accretion disk and not the biconical flow (Dietrich et al. 1998; Paper I);

2. Livio \& Xu (1997) demonstrated that the double-peaked lines seen in 3C 390.3 cannot be produced by two lineemitting streams, since the emission region of the far cone jet would be obscured by the optically thick accretion disk. The aforementioned simple scattering models obviously would be unable to explain the available polarimetric and optic observations.

The BLR in 3C 390.3 may have a complex structure and broad line fluxes that originate in several components, such as an accretion disk, a spherical ensemble of clouds following virilized orbits, a bipolar radial outflow, or pc-jet.

\section{Conclusion}

We have presented a long-term spectroscopic monitoring campaign of 3C 390.3. We have constructed the continuum and $\mathrm{H} \alpha$ and $\mathrm{H} \beta$ light curves, performed cross correlation between the continuum flux and line fluxes as well as between the line parts. In addition, we have analyzed any changes in $\mathrm{H} \beta$ and $\mathrm{H} \alpha$ line profiles and compiled mean and rms profiles. From our analysis and inspection of the observed data, we have reached the following conclusions:

(i) During 1995-2007, the $\mathrm{H} \alpha$ and $\mathrm{H} \beta$ broad emission lines and continuum varied 3-5 times. The amplitude of variability $F$ (var) was larger in the continuum (0.46) than in either $\mathrm{H} \beta$ (0.38) or $\mathrm{H} \alpha$ (0.35). The blue $\mathrm{H} \beta$ and $\mathrm{H} \alpha$ wings were always brighter and vary slightly more strongly than those in the red. The $\mathrm{H} \alpha$ and $\mathrm{H} \beta$ profiles varied in a way 

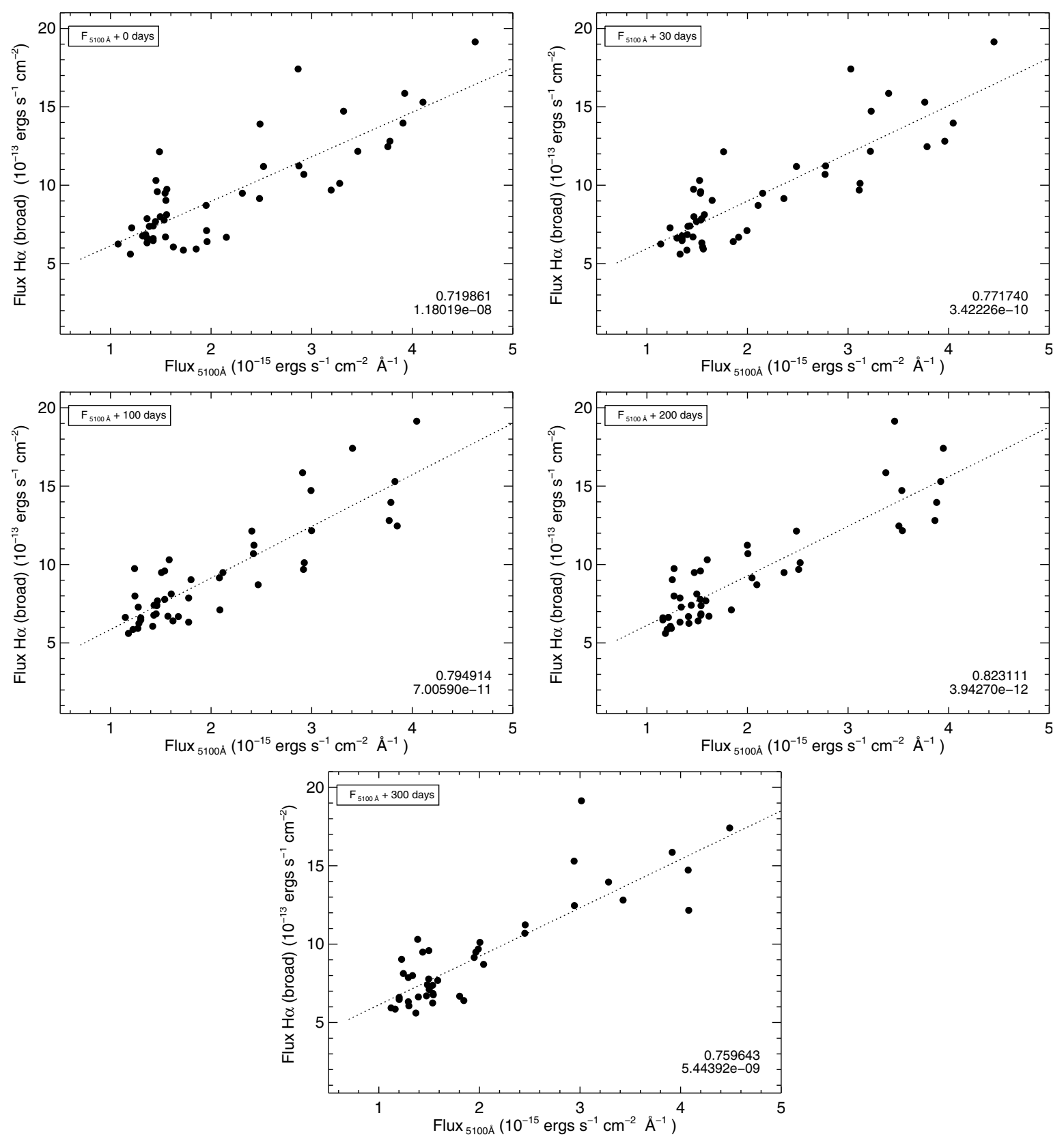

Fig. 20. $\mathrm{H} \alpha$ vs. continuum correlations - with and without lags. Shifts for the continuum are given in the top left and the correlation coefficient and the $p$-value are in the bottom right.

similar to those of Sy 1 type in the maximum activity state and Sy1.8 type in the minimum activity state.

(ii) We found that QPOs exist in the $\mathrm{H} \beta$ and continuum light curve, as commonly observed for stellar-mass black holes. The periodicity of the light curves is probably connected with some shock waves near the supermassive black hole spreading in the outer part of the disk, but on the other hand, we cannot exclude the contribution of either ejection or jets to QPOs. Our results imply that the QPO variations in the observed flux ratio of the blue to red $\mathrm{H} \beta$ wings, of period $\sim 10$ years $(P \sim 10 \mathrm{yr})$, probably exist, as previously detected by Veilleux \& Zheng (1991).

(iii) Cross-correlation of the continuum and $\mathrm{H} \beta$ broad emission line fluxes shows a lag $~ 95$ days, as in both Paper I (in 1998-1999, 100 days) and Sergeev et al. (2002, lag $\sim 89$ days). There is no significant lag between both the blue and red wings and the core, which is indicative of predominantly circular motions in the BLR of 3C 390.3 and a disk-like geometry. For $\mathrm{H} \alpha$, we found a lag of around 120 days. This difference in lags as well as in the FWHM of $\mathrm{H} \alpha$ and $\mathrm{H} \beta$ may imply that there is some stratification in the BLR (disk) of 3C 390.3.

(iv) The $\mathrm{H} \alpha$ and $\mathrm{H} \beta$ line fluxes and parts of lines are strongly correlated with the continuum flux, indicating that the ionizing continuum was a good extrapolation of the optical one.

(v) By comparing the $\mathrm{H} \beta$ and $\mathrm{H} \alpha$ line profiles, we found that the broad emission region has a disk-like structure, but there probably exists a non-disk component, or also a disklike one of different parameters that contributes to the line emission. More details about the line shape variation and any possible disk structure will be given in a forthcoming paper. 
Acknowledgements. This work was supported by INTAS (grant N96-0328), RFBR (grants N97-02-17625 N00-02-16272, N03-02-17123, 06-02-16843, and N09-02-01136), State program "Astronomy" (Russia), CONACYT research grant 39560-F and 54480 (México), PROMEP/103.5/08/4722 grant and the Ministry of Science and Technological Development of Republic of Serbia through the project Astrophysical Spectroscopy of Extragalactic Objects (146002). L.C.P., W.K. and D.I. are grateful to the Alexander von Humboldt foundation for support in the frame of program "Research Group Linkage". We would like to thank to the anonymous referee for very useful comments.

\section{References}

Alef, W., Gotz, M. M. A., Preuss, E., \& Kellermann, K. I. 1988, A\&A, 192, 53 Alexander, T. 1997, Astronomical Time Series, ed. D. Maoz, A. Sternberg, \& E. M. Leibowitz (Dordrecht: Kluwer), 163

Alef, W., Wu, S. Y., Preuss, E., Kellermann, K. I., \& Qiu, Y. H. 1996, A\&A, 308, 376

Allen, C. W. 1973, Astrophysical Quantities (London: Athlone)

Arshakian, T. G., León-Tavares, J., Lobanov, A. P., et al. 2010, MNRAS, 401, 1231

Barr, P., Pollard, G., Sanford, P. W., et al. 1980, MNRAS, 193, 549

Barr, P., Willis, A. J., \& Wilson, R. 1983, MNRAS, 202, 453

Baum, S. A., Heckman, T., Bridle, A., van Breugel, W., \& Miley, G. 1988, ApJS, 68,643

Benitez, E., Chavushyan, V. H., Raiteri, C. M., et al. 2009, Proceeding Accretion and Ejection in AGN: A Global View [arXiv: 0910.0437]

Bischoff, K., \& Kollatschny, W. 1999, A\&A, 345, 49

Bochkarev, N. G., Burenkov, A. N., \& Shapovalova, A. I. 1997, Astron. Astrophys. Trans., 14, 97

Bon, E., Popović, L. Č., Gavrilović, N., La Mura, G., \& Mediavilla, E. 2009, MNRAS, 400, 924

Clavel, J. C., \& Wamsteker, W. 1987, ApJ, 320, L9

Chakrabarti, S. K., \& Wiita, P. J. 1994, ApJ, 434, 518

Chakrabarti, S. E., Acharyya, K., \& Molteni, D. 2004, A\&A, 421, 1

Corbett, E. A., Robinson, A., Axon, D. J., Young, S., \& Hough, J. H. 1998, MNRAS, 296, 721

Corbett, E. A., Robinson, A., Axon, D. J., \& Young, S. 2000, MNRAS, 319, 685

Dietrich, M., Peterson, B. M., Albrecht, P., et al. 1998, ApJS, 115, 185

Eracleous, M., Livio, M., Halpern, J. P., \& Storchi-Bergmann, T. 1995, ApJ, 438, 610

Eracleous, M., Halpern, J. P., \& Livio, M. 1996, ApJ, 459, 89

Gaskell, C. M. 1996, ApJ, 464, L107

Gezari, S., Halpern, J. P., \& Eracleous, M. 2007, ApJS, 169, 167

Gierliński, M., Middleton, M., Ward, M., \& Done, C. 2008, Nature, 455, 369

Gliozzi, M., Sambruna, R. M., \& Eracleous, M. 2003, ApJ, 584, 176

Gupta, A. C., Srivastava, A. K., \& Wiita, P. J. 2009, ApJ, 690, 216
Harris, D. E., Cheung, C. C., Stawarz, L., Biretta, J. A., \& Perlman, E. S. 2009, ApJ, 699, 305

Hovatta, T., Lehto, H. J., \& Tornikoski, M. 2008, A\&A, 488, 897

Inda, M., Makishima, K., Kohmura, Y., et al. 1994, ApJ, 420, 143

Lachowicz, P., Gupta, A. C., Gaur, H., \& Wiita, P. J. 2009, A\&A, 506, L17

Leahy, J. P., \& Perley, R. A. 1991, AJ, 102, 537

Leahy, J. P., \& Perley, R. A. 1995, MNRAS, 277,1097

Leighly, K. M., O’Brien, P. T., Edelson, R., et al. 1997, ApJ, 483, 767

León-Tavares, J., Lobanov, A. P., Chavushyan, V. H., et al. 2010, ApJ, 715, 355

Livio, M., \& Xu, C. 1997, Apj, 478, L63

Middleton, M., Done, C., Ward, M., Gierliński, M., \& Schurch, N. 2009, MNRAS, 394, 250

Netzer, H. 1982, MNRAS, 198, 589

O’Brien, P. T., Dietrich, M., Leighly, K., et al. 1998, ApJ, 509, 163

Osterbrock, D. E., Koski, A. T., \& Phillips, M. M. 1975, ApJ, 197, L41

Penston, M. V., \& Perez, E. 1984, MNRAS, 211, 13P

Perez, E., Mediavilla, E., Penston, M. V., Tadhunter, C., \& Moles, M. 1988, MNRAS, 230, 353

Peterson, B. M. 1993, PASP, 105, 207

Peterson, B. M. 1999, ASPC, 175, 49

Peterson, B. M., \& Collins II, G. W. 1983, ApJ, 270, 71

Peterson, B. M., Pogge, R. W., Wanders, I., Smith, S. M., \& Romanishin, W. 1995, PASP, 107, 579

Popović, L. Č., Mediavilla, E., Bon, E., \& Ilić, D. 2004, A\&A, 423, 909

Rokaki, E., Boisson, C., \& Collin-Souffrin, S. 1992, A\&A, 253, 57

Schield, R. E., 1983, PASP, 95, 1021

Sergeev, S. G., Pronik, V. I., Peterson, B. M., Sergeeva, E. A., \& Zheng, W. 2002, ApJ, 576, 660S

Shapovalova, A. I., Burenkov, A. N., \& Bochkarev, N. G. 1996, Bull. Spec. Astrophys. Obs., 41, 28

Shapovalova, A. I., Burenkov, A. N., Carrasco, L., et al. 2001, A\&A, 376, 775

Shapovalova, A. I., Doroshenko, V. T., Bochkarev, N. G., et al. 2004, A\&A, 422, 925

Shapovalova, A. I., Popović, L.Č., Collin, S., et al. 2008, A\&A, 486, 99

Shapovalova, A. I., Popović, L.Č., Bochkarev, N. G., et al. 2009, NewAR, 53, 191

Smith, J. E., Robinson, A., Alexander, D. M., et al. 2004, MNRAS, 350, 140

Smith, J. E., Robinson, A., Young, S., Axon, D. J., \& Corbett, E. A. 2005, MNRAS, 359, 846

Tao, J., Fan, J., Qian, B., \& Liu, Y. 2008, AJ, 135, 737

Torrence, C., \& Compo, G. P. 1998, Bull. Amer. Meteor. Soc., 79, 61

Van Groningen, E., \& Wanders, I. 1992, PASP, 104, 700

Veilleux, S., \& Zheng, W. 1991, ApJ, 377, 89

Wamsteker W. 1981, A\&A, 97, 329

Wamsteker, W., Wang, T.-G., Schartel, N., \& Vio, R. 1997, MNRAS, 288, 225

Wozniak, P. R., Zdziarski, A. A., Smith, D., Madejski, G. M., \& Johnson, W. N. 1998, MNRAS, 299, 449

Yee, H. K., \& Oke, J. B. 1981, ApJ, 248, 472

Zheng, W. 1996, AJ, 111, 1498 
Table 1. The log of spectroscopic observations.

\begin{tabular}{|c|c|c|c|c|c|c|c|}
\hline$N$ & UT-date & $\begin{array}{c}\text { JD } \\
2400000+\end{array}$ & CODE $^{*}$ & $\begin{array}{l}\text { Aperture } \\
\text { arcsec }\end{array}$ & $\begin{array}{c}\text { Sp.range } \\
\AA\end{array}$ & $\begin{array}{c}\text { Res } \\
\AA\end{array}$ & $\begin{array}{l}\text { Seeing } \\
\operatorname{arcsec}\end{array}$ \\
\hline 1 & 2 & 3 & 4 & 5 & 6 & 7 & 8 \\
\hline 1 & 1995 Apr. 25 & 49832.424 & $\mathrm{~L}(\mathrm{G})$ & $3.0 \times 3.6$ & $4000-5400$ & - & 2 \\
\hline 2 & 1995 May 26 & 49863.375 & $\mathrm{~L}(\mathrm{~T})$ & $3.6 \times 3.6$ & $4900-5450$ & 4.5 & 2 \\
\hline 3 & 1995 Nov. 17 & 50039.156 & $\mathrm{~L}(\mathrm{G})$ & $3.6 \times 8.4$ & $3700-5800$ & - & - \\
\hline 4 & 1995 Nov. 29 & 50051.143 & $\mathrm{~L}(\mathrm{G})$ & $2.0 \times 8.4$ & $4600-5400$ & - & - \\
\hline 5 & 1995 Nov. 30 & 50052.149 & $\mathrm{~L}(\mathrm{G})$ & $2.0 \times 10.8$ & $4700-5600$ & - & - \\
\hline 6 & 1996 Feb. 14 & 50127.602 & $\mathrm{~L}(\mathrm{~N})$ & $1.5 \times 6.0$ & $3100-5800$ & 6 & 2 \\
\hline 7 & 1996 Mar. 20 & 50162.580 & $\mathrm{~L}(\mathrm{~N})$ & $2.0 \times 6.0$ & $3600-5600$ & 6 & 3 \\
\hline 8 & 1996 Mar. 20 & 50162.587 & $\mathrm{~L}(\mathrm{~N})$ & $2.0 \times 6.0$ & $4700-7400$ & 6 & 3 \\
\hline 9 & 1996 Mar. 21 & 50163.553 & $\mathrm{~L}(\mathrm{~N})$ & $2.0 \times 6.0$ & $3600-5600$ & 6 & 3 \\
\hline 10 & 1996 Mar. 21 & 50163.572 & $\mathrm{~L}(\mathrm{~N})$ & $2.0 \times 6.0$ & $4700-7400$ & 6 & 3 \\
\hline 11 & 1996 Jun. 15 & 50249.542 & $\mathrm{~L}(\mathrm{G})$ & $2.0 \times 6.0$ & $4600-5500$ & 5 & 2.5 \\
\hline 12 & 1996 Jul. 12 & 50276.567 & $\mathrm{~L}(\mathrm{G})$ & $2.0 \times 6.0$ & $4600-5500$ & 5 & 1.4 \\
\hline 13 & 1996 Jul. 12 & 50276.570 & $\mathrm{~L}(\mathrm{G})$ & $2.0 \times 6.0$ & $6370-7270$ & 5 & 1.4 \\
\hline 14 & 1996 Jul. 13 & 50277.556 & $\mathrm{~L}(\mathrm{G})$ & $2.0 \times 6.0$ & $4600-5500$ & 5 & 1.6 \\
\hline 15 & 1996 Jul. 17 & 50281.434 & $\mathrm{~L}(\mathrm{G})$ & $2.0 \times 6.0$ & $4100-5800$ & 8 & 1.3 \\
\hline 16 & 1996 Aug. 10 & 50305.489 & $\mathrm{~L}(\mathrm{G})$ & $2.0 \times 6.0$ & $4600-5575$ & 5 & 3.2 \\
\hline 17 & 1996 Sep. 11 & 50338.800 & $\mathrm{Z} 2$ & $4.2 \times 13.8$ & $3850-5725$ & 5 & 5 \\
\hline 18 & 1996 Nov. 03 & 50390.431 & $\mathrm{~L}(\mathrm{G})$ & $2.0 \times 6.0$ & $4600-5500$ & 4 & 3 \\
\hline 19 & 1996 Nov. 05 & 50392.621 & $\mathrm{~L}(\mathrm{G})$ & $2.0 \times 6.0$ & $4621-5521$ & 4 & $3-4$ \\
\hline 20 & 1997 Mar. 04 & 50511.622 & $\mathrm{~L}(\mathrm{G})$ & $2.0 \times 6.0$ & $4650-5550$ & 5 & 1.8 \\
\hline 21 & 1997 May 30 & 50599.370 & $\mathrm{~L}(\mathrm{G})$ & $2.0 \times 6.0$ & $4700-5600$ & 5 & 2.5 \\
\hline 22 & 1997 Jul. 26 & 50656.499 & $\mathrm{~L}(\mathrm{G})$ & $2.0 \times 6.0$ & $4700-5675$ & 5 & $3-4$ \\
\hline 23 & 1997 Aug. 30 & 50691.463 & $\mathrm{~L}(\mathrm{G})$ & $2.0 \times 6.0$ & $4700-5600$ & 5 & 2 \\
\hline 24 & 1997 Sep. 10 & 50701.576 & $\mathrm{~L}(\mathrm{~N})$ & $2.0 \times 6.0$ & $3900-6600$ & 6 & - \\
\hline 25 & 1997 Sep. 10 & 50701.566 & $\mathrm{~L}(\mathrm{~N})$ & $2.0 \times 6.0$ & $5200-7900$ & 6 & - \\
\hline 26 & 1997 Dec. 26 & 50808.582 & $\mathrm{~L}(\mathrm{U})$ & $2.0 \times 6.0$ & $4800-5700$ & 4 & 3.0 \\
\hline 27 & 1997 Dec. 27 & 50810.184 & $\mathrm{~L}(\mathrm{U})$ & $2.0 \times 6.0$ & $4750-5650$ & 4 & 4.0 \\
\hline 28 & 1997 Dec. 27 & 50810.203 & $\mathrm{~L}(\mathrm{U})$ & $2.0 \times 6.0$ & $6400-7300$ & 4 & 4.0 \\
\hline 29 & 1997 Dec. 28 & 50811.194 & $\mathrm{~L}(\mathrm{U})$ & $2.0 \times 6.0$ & $4750-5650$ & 4 & 2.6 \\
\hline 30 & 1997 Dec. 28 & 50811.206 & $\mathrm{~L}(\mathrm{U})$ & $2.0 \times 6.0$ & $6400-7300$ & 4 & 2.6 \\
\hline 31 & 1997 Dec. 30 & 50813.195 & $\mathrm{~L}(\mathrm{G})$ & $2.0 \times 6.0$ & $3600-7200$ & 12 & 3.2 \\
\hline 32 & 1998 Jan. 22 & 50835.631 & $\mathrm{~L}(\mathrm{~N})$ & $2.0 \times 6.0$ & $3800-6200$ & 6 & 2 \\
\hline 33 & 1998 Feb. 23 & 50867.560 & $\mathrm{~L}(\mathrm{~N})$ & $2.0 \times 6.0$ & $3800-6200$ & 6 & 2 \\
\hline 34 & 1998 Feb. 23 & 867.573 & $\mathrm{~L}(\mathrm{~N})$ & $2.0 \times 6.0$ & $5000-7400$ & 6 & 2 \\
\hline 35 & 1998 May 06 & 50940.354 & $\mathrm{~L}(\mathrm{~N})$ & $2.0 \times 6.0$ & $3700-6200$ & 6 & 3 \\
\hline 36 & 1998 Jun. 25 & 50990.302 & $\mathrm{~L}(\mathrm{~N})$ & $2.0 \times 6.0$ & $3600-6100$ & 6 & 3 \\
\hline 37 & 1998 Jul. 16 & 51010.719 & GHO & $2.5 \times 6.0$ & $4200-7400$ & 15 & 2 \\
\hline 38 & 1998 Jul. 25 & 51019.723 & $\mathrm{GHO}$ & $2.5 \times 6.0$ & $4440-7640$ & 15 & 2.2 \\
\hline 39 & 1998 Jul. 28 & 51023.491 & $\mathrm{~L}(\mathrm{G})$ & $2.0 \times 6.0$ & $4800-5700$ & 5 & 1.3 \\
\hline 40 & 1998 Jul. 30 & 51025.431 & $\mathrm{~L}(\mathrm{G})$ & $2.0 \times 6.0$ & $4800-5700$ & 5 & 2 \\
\hline 41 & 1998 Aug. 30 & 51055.551 & $\mathrm{~L}(\mathrm{~N})$ & $2.0 \times 6.0$ & $4000-6500$ & 6 & 1.8 \\
\hline 42 & 1998 Aug. 30 & 51055.562 & $\mathrm{~L}(\mathrm{~N})$ & $2.0 \times 6.0$ & $5250-7750$ & 6 & 1.7 \\
\hline 43 & 1998 Sep. 19 & 51076.004 & $\mathrm{Z} 1$ & $4.2 \times 19.8$ & $4100-5900$ & 8 & 2.0 \\
\hline 44 & 1998 Sep. 25 & 51081.429 & $\mathrm{GHO}$ & $2.5 \times 6.0$ & $4230-7430$ & 15 & 2 \\
\hline 45 & 1998 Sep. 26 & 51082.429 & $\mathrm{GHO}$ & $2.5 \times 6.0$ & $4210-7410$ & 15 & 2 \\
\hline 46 & 1998 Sep. 27 & 51083.429 & GHO & $2.5 \times 6.0$ & $4210-7410$ & 15 & 2 \\
\hline 47 & 1998 Oct. 25 & 51112.259 & $\mathrm{~L}(\mathrm{G})$ & $2.0 \times 6.0$ & $4700-5600$ & 5 & 3 \\
\hline 48 & 1998 Dec. 02 & 51149.625 & GHO & $2.5 \times 6.0$ & $4220-7600$ & 15 & - \\
\hline 49 & 1999 Jul. 13 & 51372.516 & $\mathrm{~L}(\mathrm{P})$ & $2.0 \times 6.0$ & $4420-5655$ & 4 & 1.5 \\
\hline 50 & 1999 Aug. 19 & 51410.309 & $\mathrm{~L}(\mathrm{P})$ & $2.0 \times 6.0$ & $4500-5740$ & 4 & 2 \\
\hline 51 & 1999 Sep. 04 & 51425.904 & $\mathrm{~L}(\mathrm{U})$ & $2.0 \times 6.0$ & $3600-6024$ & 7 & 1.5 \\
\hline 52 & 1999 Oct. 03 & 51454.674 & GHO & $2.5 \times 6.0$ & $4060-7330$ & 15 & 2 \\
\hline 53 & 1999 Sep. 05 & 51427.272 & $\mathrm{~L}(\mathrm{P})$ & $2.0 \times 6.0$ & $4900-7320$ & 7 & 1.5 \\
\hline 54 & 1999 Oct. 04 & 51455.664 & $\mathrm{~L}(\mathrm{U})$ & $2.0 \times 6.0$ & $4480-5728$ & 4 & 1.3 \\
\hline 55 & 1999 Oct. 09 & 51461.531 & $\mathrm{~L}(\mathrm{~N})$ & $2.0 \times 6.0$ & $4250-6580$ & 8 & 3 \\
\hline 56 & 2000 Jul. 19 & 51745.365 & $\mathrm{~L}(\mathrm{U})$ & $2.0 \times 6.0$ & $4390-5590$ & 5 & 2.2 \\
\hline 57 & 2000 Jul. 22 & 51748.232 & $\mathrm{~L}(\mathrm{U})$ & $2.0 \times 6.0$ & $4390-5580$ & 5 & - \\
\hline 58 & 2000 Jul. 30 & 51756.293 & $\mathrm{~L}(\mathrm{U})$ & $2.0 \times 6.0$ & $3690-6090$ & 8 & 1.6 \\
\hline
\end{tabular}


A. I. Shapovalova et al.: Spectral monitoring of 3C 390.3. I.

Table 1. continued.

\begin{tabular}{|c|c|c|c|c|c|c|c|}
\hline $\mathrm{N}$ & UT-date & $\begin{array}{c}\text { JD } \\
2400000+\end{array}$ & CODE $^{*}$ & $\begin{array}{l}\text { Aperture } \\
\text { arcsec }\end{array}$ & $\begin{array}{c}\text { Sp.range } \\
\AA\end{array}$ & $\begin{array}{c}\text { Res } \\
\AA\end{array}$ & $\begin{array}{l}\text { Seeing } \\
\text { arcsec } \\
\Omega\end{array}$ \\
\hline 1 & 2 & 3 & 4 & 5 & 6 & 7 & 8 \\
\hline 59 & 2000 Jul. 30 & 51756.317 & $\mathrm{~L}(\mathrm{U})$ & $2.0 \times 6.0$ & $5640-8040$ & 8 & 1.6 \\
\hline 60 & 2000 Oct. 05 & 51823.142 & $\mathrm{~L}(\mathrm{U})$ & $2.0 \times 6.0$ & $3690-6030$ & 8 & 1.2 \\
\hline 61 & 2000 Nov. 18 & 51867.136 & $\mathrm{~L}(\mathrm{U})$ & $2.0 \times 6.0$ & $4490-5690$ & 5 & 1.0 \\
\hline 62 & 2000 Nov. 19 & 51868.128 & $\mathrm{~L}(\mathrm{U})$ & $2.0 \times 6.0$ & $4490-5690$ & 5 & 1.2 \\
\hline 63 & 2001 Jan. 16 & 51925.631 & $\mathrm{~L}(\mathrm{U})$ & $2.0 \times 6.0$ & $3690-6040$ & 8 & 1.8 \\
\hline 64 & 2001 Jan. 16 & 51925.647 & $\mathrm{~L}(\mathrm{U})$ & $2.0 \times 6.0$ & $5690-8040$ & 8 & 1.8 \\
\hline 65 & 2001 Jan. 20 & 51929.623 & $\mathrm{~L}(\mathrm{U})$ & $2.0 \times 6.0$ & $3690-6040$ & 8 & 1.8 \\
\hline 66 & 2001 Jan. 20 & 51929.639 & $\mathrm{~L}(\mathrm{U})$ & $2.0 \times 6.0$ & $5740-7990$ & 8 & 1.8 \\
\hline 67 & 2001 Mar. 13 & 51981.950 & $\mathrm{GHO}$ & $2.5 \times 6.0$ & $4116-7436$ & 15 & 2.0 \\
\hline 68 & 2001 May 05 & 52034.660 & $\mathrm{GHO}$ & $2.5 \times 6.0$ & $4160-7470$ & 15 & - \\
\hline 69 & 2001 May 14 & 52043.900 & $\mathrm{GHO}$ & $2.5 \times 6.0$ & $3980-7280$ & 15 & - \\
\hline 70 & 2001 Jun. 14 & 52074.850 & GHO & $2.5 \times 6.0$ & $4010-7320$ & 15 & - \\
\hline 71 & 2001 Jun. 14.5 & 52075.350 & $\mathrm{GHO}$ & $2.5 \times 6.0$ & $4010-7320$ & 15 & - \\
\hline 72 & 2001 Jun. 15 & 52075.850 & $\mathrm{GHO}$ & $2.5 \times 6.0$ & $4010-7320$ & 15 & - \\
\hline 73 & 2001 Oct. 10 & 52192.600 & $\mathrm{GHO}$ & $2.5 \times 6.0$ & $4030-7336$ & 15 & 2.3 \\
\hline 74 & 2001 Nov. 23 & 52237.272 & $\mathrm{~L}(\mathrm{U})$ & $2.0 \times 6.0$ & $3620-6000$ & 8 & 3.5 \\
\hline 75 & 2002 Feb. 21 & 52327.200 & L(U) & $2.0 \times 6.0$ & $5690-8040$ & 8 & 2.5 \\
\hline 76 & 2002 Feb. 21 & 52327.226 & $\mathrm{~L}(\mathrm{U})$ & $2.0 \times 6.0$ & $3490-5840$ & 8 & 2.5 \\
\hline 77 & 2002 Mar. 05 & 52339.019 & $\mathrm{GHO}$ & $2.5 \times 6.0$ & $3880-7190$ & 15 & 2.0 \\
\hline 78 & 2002 Apr. 03 & 52368.005 & $\mathrm{GHO}$ & $2.5 \times 6.0$ & $4290-5960$ & 8 & - \\
\hline 79 & 2002 Jun. 03 & 52428.968 & GHO & $2.5 \times 6.0$ & $5740-7430$ & 8 & - \\
\hline 80 & 2002 Jun. 04 & 52429.950 & $\mathrm{GHO}$ & $2.5 \times 6.0$ & $3980-7300$ & 15 & - \\
\hline 81 & 2002 Jun. 05 & 52430.938 & GHO & $2.5 \times 6.0$ & $4240-5910$ & 8 & - \\
\hline 82 & 2002 Jun. 24 & 52450.385 & $\mathrm{~L}(\mathrm{U})$ & $2.0 \times 6.0$ & $3500-5840$ & 8 & 5 \\
\hline 83 & 2002 Jun. 24 & 52450.410 & $\mathrm{~L}(\mathrm{U})$ & $2.0 \times 6.0$ & $5640-7990$ & 8 & 5 \\
\hline 84 & 2002 Jul. 09 & 52464.444 & $\mathrm{~L}(\mathrm{U})$ & $2.0 \times 6.0$ & $3500-5840$ & 8 & 2 \\
\hline 85 & 2002 Jul. 13 & 52469.408 & $\mathrm{~L}(\mathrm{U})$ & $2.0 \times 6.0$ & $3500-5840$ & 8 & 2 \\
\hline 86 & 2002 Jul. 13 & 52469.433 & $\mathrm{~L}(\mathrm{U})$ & $2.0 \times 6.0$ & $5640-7990$ & 8 & 2 \\
\hline 87 & 2002 Aug. 08 & 52495.272 & $\mathrm{~L}(\mathrm{U})$ & $2.0 \times 6.0$ & $4290-5450$ & 8 & - \\
\hline 88 & 2002 Aug. 16 & 52502.795 & GHO & $2.5 \times 6.0$ & $4250-5930$ & 8 & - \\
\hline 89 & 2002 Oct. 14 & 52562.318 & $\mathrm{~L}(\mathrm{U})$ & $2.0 \times 6.0$ & $5640-7990$ & 8 & 2 \\
\hline 90 & 2002 Oct. 14 & 52562.342 & $\mathrm{~L}(\mathrm{U})$ & $2.0 \times 6.0$ & $3500-5840$ & 8 & 2 \\
\hline 91 & 2002 Nov. 13 & 52591.659 & $\mathrm{GHO}$ & $2.5 \times 6.0$ & $5720-7410$ & 8 & - \\
\hline 92 & 2002 Nov. 14 & 52592.659 & $\mathrm{GHO}$ & $2.5 \times 6.0$ & $5720-7410$ & 8 & - \\
\hline 93 & 2002 Nov. 15 & 52593.671 & $\mathrm{GHO}$ & $2.5 \times 6.0$ & $3800-7115$ & 15 & - \\
\hline 94 & 2002 Dec. 11 & 52620.389 & $\mathrm{~L}(\mathrm{U})$ & $2.0 \times 6.0$ & $3650-5990$ & 8 & 2 \\
\hline 95 & 2003 May 08 & 52768.217 & $\mathrm{~L}(\mathrm{U})$ & $2.0 \times 6.0$ & $3700-6040$ & 8 & 1.5 \\
\hline 96 & 2003 May 10 & 52770.219 & $\mathrm{~L}(\mathrm{U})$ & $2.0 \times 6.0$ & $5750-8090$ & 8 & 1.5 \\
\hline 97 & 2003 May 23 & 52782.966 & GHO & $2.5 \times 6.0$ & $3540-7165$ & 15 & - \\
\hline 98 & 2003 May 24 & 52783.976 & $\mathrm{GHO}$ & $2.5 \times 6.0$ & $4220-6060$ & 8 & - \\
\hline 99 & 2003 May 25 & 52784.973 & GHO & $2.5 \times 6.0$ & $5580-7440$ & 8 & - \\
\hline 100 & 2003 May 26 & 52785.979 & $\mathrm{GHO}$ & $2.5 \times 6.0$ & $4230-6040$ & 8 & - \\
\hline 101 & 2003 Jun. 22 & 52812.963 & $\mathrm{GHO}$ & $2.5 \times 6.0$ & $4270-5980$ & 8 & - \\
\hline 102 & 2003 Sep. 18 & 52900.794 & $\mathrm{GHO}$ & $2.5 \times 6.0$ & $3710-7100$ & 15 & - \\
\hline 103 & 2003 Sep. 20 & 52902.803 & $\mathrm{GHO}$ & $2.5 \times 6.0$ & $5630-7340$ & 8 & - \\
\hline 104 & 2003 Oct. 21 & 52933.672 & GHO & $2.5 \times 6.0$ & $3840-7220$ & 15 & - \\
\hline 105 & 2003 Nov. 19 & 52962.637 & $\mathrm{GHO}$ & $2.5 \times 6.0$ & $3750-7132$ & 15 & - \\
\hline 106 & 2003 Nov. 20 & 52963.608 & GHO & $2.5 \times 6.0$ & $4240-5950$ & 8 & - \\
\hline 107 & 2004 Mar. 01 & 53066.358 & $\mathrm{~L}(\mathrm{U})$ & $2.0 \times 6.0$ & $5800-8100$ & 8 & 2 \\
\hline 108 & 2004 Mar. 01 & 53065.507 & $\mathrm{~L}(\mathrm{U})$ & $2.0 \times 6.0$ & $3740-6090$ & 8 & 2 \\
\hline 109 & 2004 May 20 & 53145.982 & $\mathrm{GHO}$ & $2.5 \times 6.0$ & $4191-5910$ & 8 & - \\
\hline 110 & 2004 Jun. 12 & 53168.955 & $\mathrm{GHO}$ & $2.5 \times 6.0$ & $4210-5920$ & 8 & - \\
\hline 111 & 2004 Jun. 13 & 53169.966 & $\mathrm{GHO}$ & $2.5 \times 6.0$ & $5570-7320$ & 8 & - \\
\hline 112 & 2004 Jun. 15 & 53171.944 & GHO & $2.5 \times 6.0$ & $3760-7148$ & 15 & - \\
\hline 113 & 2004 Aug. 19 & 53236.808 & $\mathrm{GHO}$ & $2.5 \times 6.0$ & $4280-5940$ & 8 & - \\
\hline 114 & 2004 Aug. 20 & 53237.821 & $\mathrm{GHO}$ & $2.5 \times 6.0$ & $5630-7360$ & 8 & - \\
\hline 115 & 2004 Aug. 21 & 53238.858 & GHO & $2.5 \times 6.0$ & $3700-7093$ & 15 & - \\
\hline 116 & 2004 Sep. 06 & 53254.771 & $\mathrm{GHO}$ & $2.5 \times 6.0$ & $4190-5900$ & 8 & - \\
\hline 117 & 2004 Dec. 18 & 53358.140 & $\mathrm{~L}(\mathrm{~S})$ & $1.0 \times 6.1$ & $3100-7300$ & 10 & 4.5 \\
\hline
\end{tabular}


Table 1. continued.

\begin{tabular}{|c|c|c|c|c|c|c|c|}
\hline $\mathrm{N}$ & UT-date & $\begin{array}{c}\text { JD } \\
2400000+\end{array}$ & CODE $^{*}$ & $\begin{array}{l}\text { Aperture } \\
\text { arcsec }\end{array}$ & $\begin{array}{c}\text { Sp.range } \\
\AA\end{array}$ & $\begin{array}{c}\text { Res } \\
\AA\end{array}$ & $\begin{array}{l}\text { Seeing } \\
\text { arcsec }\end{array}$ \\
\hline 1 & 2 & 3 & 4 & 5 & 6 & 7 & 8 \\
\hline 118 & 2004 Dec. 19 & 53359.130 & $\mathrm{~L}(\mathrm{~S})$ & $1.0 \times 6.1$ & $3100-7300$ & 10 & 2.0 \\
\hline 119 & 2004 Dec. 20 & 53360.150 & $\mathrm{~L}(\mathrm{~S})$ & $1.0 \times 6.1$ & $3100-7300$ & 10 & 1.6 \\
\hline 120 & 2005 Apr. 15 & 53475.997 & GHO & $2.5 \times 6.0$ & $4250-5960$ & 8 & - \\
\hline 121 & 2005 Apr. 16 & 53476.993 & GHO & $2.5 \times 6.0$ & $5540-7250$ & 8 & - \\
\hline 122 & 2005 Apr. 18 & 53478.992 & GHO & $2.5 \times 6.0$ & $3790-7173$ & 15 & - \\
\hline 123 & 2005 May 13 & 53503.972 & GHO & $2.5 \times 6.0$ & $4220-5910$ & 8 & - \\
\hline 124 & 2005 Jun. 10 & 53531.925 & GHO & $2.5 \times 6.0$ & $4280-5970$ & 8 & - \\
\hline 125 & 2005 Aug. 30 & 53612.758 & GHO & $2.5 \times 6.0$ & $4330-5990$ & 8 & - \\
\hline 126 & 2005 Aug. 31 & 53613.773 & GHO & $2.5 \times 6.0$ & $4330-6000$ & 8 & - \\
\hline 127 & 2005 Sep. 01 & 53614.750 & GHO & $2.5 \times 6.0$ & $4330-6000$ & 8 & - \\
\hline 128 & 2005 Dec. 06 & 53711.420 & $\mathrm{~L}(\mathrm{~S})$ & $1.0 \times 6.1$ & $3100-7300$ & 10 & 4.0 \\
\hline 129 & 2006 Jun. 04 & 53891.490 & $\mathrm{~L}(\mathrm{~S})$ & $1.0 \times 6.1$ & $3100-7300$ & 10 & 1.5 \\
\hline 130 & 2006 Sep. 01 & 53979.790 & GHO & $2.5 \times 6.0$ & $3500-7044$ & 15 & 2.1 \\
\hline 131 & 2006 Sep. 16 & 53994.780 & GHO & $2.5 \times 6.0$ & $3530-7070$ & 15 & 3.4 \\
\hline 132 & 2006 Sep. 17 & 53995.750 & GHO & $2.5 \times 6.0$ & $4150-5950$ & 7.5 & 2.4 \\
\hline 133 & 2006 Sep. 18 & 53996.700 & GHO & $2.5 \times 6.0$ & $3470-7020$ & 15 & 2.3 \\
\hline 134 & 2006 Sep. 19 & 53997.740 & GHO & $2.5 \times 6.0$ & $4120-5920$ & 7.5 & 2.4 \\
\hline 135 & 2006 Sep. 20 & 53998.750 & GHO & $2.5 \times 6.0$ & $3470-7020$ & 15 & 2.7 \\
\hline 136 & 2006 Oct. 28 & 54036.630 & GHO & $2.5 \times 6.0$ & $3710-7270$ & 15 & 2.7 \\
\hline 137 & 2006 Oct. 29 & 54037.640 & GHO & $2.5 \times 6.0$ & $4230-6030$ & 7.5 & 2.2 \\
\hline 138 & 2006 Nov. 01 & 54040.650 & GHO & $2.5 \times 6.0$ & $4158-5958$ & 7.5 & 2.3 \\
\hline 139 & 2006 Dec. 18 & 54088.210 & $\mathrm{~L}(\mathrm{~S})$ & $1.0 \times 6.1$ & $3100-7300$ & 10 & 2.0 \\
\hline 140 & 2007 Jan. 12 & 54112.640 & $\mathrm{~L}(\mathrm{~S})$ & $1.0 \times 6.1$ & $3100-7300$ & 10 & 2.9 \\
\hline 141 & 2007 Jan. 13 & 54113.650 & $\mathrm{~L}(\mathrm{~S})$ & $1.0 \times 6.1$ & $3100-7300$ & 10 & 2.2 \\
\hline 142 & 2007 Feb. 12 & 54143.610 & $\mathrm{~L}(\mathrm{~S})$ & $1.0 \times 6.1$ & $3100-7300$ & 10 & 2.3 \\
\hline 143 & 2007 May 22 & 54242.890 & GHO & $2.5 \times 6.0$ & $3745-7335$ & 15 & 3.7 \\
\hline 144 & 2007 May 23 & 54243.880 & GHO & $2.5 \times 6.0$ & $4185-5985$ & 7.5 & 4.2 \\
\hline 145 & 2007 Jun. 20 & 54271.870 & GHO & $2.5 \times 6.0$ & $3700-7150$ & 15 & 2.0 \\
\hline 146 & 2007 Jun. 21 & 54272.960 & GHO & $2.5 \times 6.0$ & $4282-6090$ & 7.5 & 2.7 \\
\hline 147 & 2007 Aug. 09 & 54321.820 & GHO & $2.5 \times 6.0$ & $3860-7430$ & 15 & 2.3 \\
\hline 148 & 2007 Aug. 11 & 54323.800 & GHO & $2.5 \times 6.0$ & $3860-7430$ & 15 & 6.6 \\
\hline 149 & 2007 Aug. 12 & 54324.810 & GHO & $2.5 \times 6.0$ & $4330-6140$ & 7.5 & 3.2 \\
\hline 150 & 2007 Sep. 03 & 54346.730 & GHO & $2.5 \times 6.0$ & $3830-7417$ & 15 & 3.4 \\
\hline 151 & 2007 Sep. 04 & 54347.720 & GHO & $2.5 \times 6.0$ & $4320-6130$ & 7.5 & 3.6 \\
\hline 152 & 2007 Sep. 05 & 54348.720 & GHO & $2.5 \times 6.0$ & $4140-5950$ & 7.5 & 2.6 \\
\hline 153 & 2007 Oct. 16 & 54389.690 & GHO & $2.5 \times 6.0$ & $3880-7440$ & 15 & 2.1 \\
\hline 154 & 2007 Oct. 18 & 54391.630 & $\mathrm{GHO}$ & $2.5 \times 6.0$ & $4200-6000$ & 7.5 & 2.5 \\
\hline 155 & 2007 Nov. 02 & 54406.650 & GHO & $2.5 \times 6.0$ & $4180-5990$ & 7.5 & 3.0 \\
\hline 156 & 2007 Nov. 04 & 54408.610 & GHO & $2.5 \times 6.0$ & $3810-7380$ & 15 & 2.9 \\
\hline 157 & 2007 Nov. 08 & 54412.690 & GHO & $2.5 \times 6.0$ & $3820-7390$ & 15 & 2.8 \\
\hline
\end{tabular}

Notes. Column (1): number. Column (2): UT date. Column (3): Julian date (JD). Column (4): CODE* . Column (5): projected spectrograph entrance apertures. Column (6): wavelength range covered. Column (7): spectral resolution. Column (8): mean seeing in arcsec.

${ }^{(*)} \mathrm{L}(\mathrm{G}, \mathrm{T}, \mathrm{U}, \mathrm{P}, \mathrm{S})-6 \mathrm{~m}$ telescope in prime focus with different variants of the long slit spectrographs and CCD; L(N) - $6 \mathrm{~m}$ telescope, Nasmyth focus; Z(1,2) - $1 \mathrm{~m}$ Zeiss, Cassegrain focus; GHO - $2.1 \mathrm{~m}$ telescope GHO (Mexico), Cassegrain focus and B\&Ch spectrograph. 
A. I. Shapovalova et al.: Spectral monitoring of 3C 390.3. I.

Table 2. Observed continuum, the broad $\mathrm{H} \alpha$ and $\mathrm{H} \beta$ fluxes, reduced to the $6 \mathrm{~m}$ telescope aperture $2^{\prime \prime} \times 6^{\prime \prime}$.

\begin{tabular}{|c|c|c|c|c|c|}
\hline $\mathrm{N}$ & UT-date & $\begin{array}{c}\text { JD } \\
2400000+ \\
3\end{array}$ & $\begin{array}{c}F(5100) \pm \sigma \\
10^{-15} \mathrm{erg} \mathrm{cm}^{-2} \mathrm{~s}^{-1} \AA^{-1} \\
4\end{array}$ & $\begin{array}{c}F(\mathrm{H} \alpha) \pm \sigma \\
10^{-13} \underset{5}{\mathrm{erg} \mathrm{cm}^{-2} \mathrm{~s}^{-1}}\end{array}$ & $\begin{array}{c}F(\mathrm{H} \beta) \pm \sigma \\
10^{-13} \mathrm{erg} \mathrm{cm}_{6}^{-2} \mathrm{~s}^{-1}\end{array}$ \\
\hline 1 & 1995 Apr. 25 & 49832.424 & $1.37 \pm 0.04$ & & $1.67 \pm 0.07$ \\
\hline 2 & 1995 May 26 & 49863.375 & $1.55 \pm 0.05$ & & $2.02 \pm 0.08$ \\
\hline 3 & 1995 Nov. 17 & 50039.156 & $2.46 \pm 0.07$ & & $2.18 \pm 0.09$ \\
\hline 4 & 1995 Nov. 29 & 50051.143 & $2.47 \pm 0.07$ & & $2.27 \pm 0.10$ \\
\hline 5 & 1995 Nov. 30 & 50052.149 & $2.55 \pm 0.08$ & & $1.89 \pm 0.08$ \\
\hline 6 & 1996 Feb. 14 & 50127.602 & $2.27 \pm 0.07$ & & $2.73 \pm 0.11$ \\
\hline 7 & 1996 Mar. 20 & 50162.580 & $2.49 \pm 0.07$ & & $2.83 \pm 0.12$ \\
\hline 8 & 1996 Mar. 20 & 50162.587 & & $13.90 \pm 1.17$ & \\
\hline 9 & 1996 Mar. 21 & 50163.553 & $2.52 \pm 0.08$ & & $3.15 \pm 0.13$ \\
\hline 10 & 1996 Mar. 21 & 50163.572 & & $11.19 \pm 0.94$ & \\
\hline 11 & 1996 Jun. 15 & 50249.542 & $1.74 \pm 0.05$ & & $2.45 \pm 0.10$ \\
\hline 12 & 1996 Jul. 12 & 50276.567 & $1.49 \pm 0.04$ & & $2.68 \pm 0.11$ \\
\hline 13 & 1996 Jul. 12 & 50276.570 & & $12.13 \pm 1.02$ & \\
\hline 14 & 1996 Jul. 13 & 50277.556 & $1.48 \pm 0.04$ & & $2.44 \pm 0.10$ \\
\hline 15 & 1996 Jul. 17 & 50281.434 & $1.57 \pm 0.05$ & & $2.38 \pm 0.10$ \\
\hline 16 & 1996 Aug. 10 & 50305.489 & $1.72 \pm 0.05$ & & $2.47 \pm 0.10$ \\
\hline 17 & 1996 Sep. 11 & 50338.800 & $1.75 \pm 0.05$ & & $1.91 \pm 0.08$ \\
\hline 18 & 1996 Nov. 03 & 50390.431 & $1.55 \pm 0.05$ & & $2.02 \pm 0.09$ \\
\hline 19 & 1996 Nov. 05 & 50392.621 & $1.50 \pm 0.05$ & & $1.84 \pm 0.08$ \\
\hline 20 & 1997 Mar. 04 & 50511.622 & $1.10 \pm 0.03$ & & $1.28 \pm 0.05$ \\
\hline 21 & 1997 May 30 & 50599.370 & $1.19 \pm 0.04$ & & $1.42 \pm 0.06$ \\
\hline 22 & 1997 Jul. 26 & 50656.499 & $0.97 \pm 0.03$ & & $1.30 \pm 0.05$ \\
\hline 23 & 1997 Aug. 30 & 50691.463 & $1.11 \pm 0.03$ & & $1.28 \pm 0.05$ \\
\hline 24 & 1997 Sep. 10 & 50701.566 & & $7.28 \pm 0.61$ & \\
\hline 25 & 1997 Sep. 10 & 50701.576 & $1.21 \pm 0.04$ & & $1.12 \pm 0.05$ \\
\hline 26 & 1997 Dec. 26 & 50808.582 & $1.68 \pm 0.05$ & & $1.53 \pm 0.06$ \\
\hline 27 & 1997 Dec. 27 & 50810.184 & $1.56 \pm 0.05$ & & $1.79 \pm 0.08$ \\
\hline 28 & 1997 Dec. 27 & 50810.203 & & $9.74 \pm 0.82$ & \\
\hline 29 & 1997 Dec. 28 & 50811.194 & $1.49 \pm 0.04$ & & $1.56 \pm 0.07$ \\
\hline 30 & 1997 Dec. 28 & 50811.206 & & $7.99 \pm 0.67$ & \\
\hline 31 & 1997 Dec. 30 & 50813.195 & $1.58 \pm 0.05$ & & $1.45 \pm 0.06$ \\
\hline 32 & 1998 Jan. 22 & 50835.631 & $1.81 \pm 0.05$ & & $1.61 \pm 0.07$ \\
\hline 33 & 1998 Feb. 23 & 50867.560 & $1.62 \pm 0.05$ & & $1.87 \pm 0.08$ \\
\hline 34 & 1998 Feb. 23 & 50867.573 & & $6.06 \pm 0.51$ & \\
\hline 35 & 1998 Мау 06 & 50940.354 & $1.87 \pm 0.06$ & & $2.29 \pm 0.10$ \\
\hline 36 & 1998 Jun. 25 & 50990.302 & $1.78 \pm 0.05$ & & $2.21 \pm 0.09$ \\
\hline 37 & 1998 Jul. 16 & 51010.719 & $1.56 \pm 0.05$ & $8.12 \pm 0.68$ & $2.35 \pm 0.10$ \\
\hline 38 & 1998 Jul. 25 & 51019.723 & $1.43 \pm 0.04$ & & $2.34 \pm 0.10$ \\
\hline 39 & 1998 Jul. 28 & 51023.491 & $1.55 \pm 0.05$ & & $2.14 \pm 0.09$ \\
\hline 40 & 1998 Jul. 30 & 51025.431 & $1.40 \pm 0.04$ & & $1.95 \pm 0.08$ \\
\hline 41 & 1998 Aug. 30 & 51055.551 & $1.45 \pm 0.04$ & & $2.04 \pm 0.09$ \\
\hline 42 & 1998 Aug. 30 & 51055.562 & & $10.30 \pm 0.87$ & \\
\hline 43 & 1998 Sep. 19 & 51076.004 & $1.65 \pm 0.05$ & & $2.07 \pm 0.09$ \\
\hline 44 & 1998 Sep. 25 & 51081.429 & $1.51 \pm 0.05$ & & $2.10 \pm 0.09$ \\
\hline 45 & 1998 Sep. 26 & 51082.429 & $1.59 \pm 0.05$ & & $2.14 \pm 0.09$ \\
\hline 46 & 1998 Sep. 27 & 51083.429 & $1.55 \pm 0.05$ & $6.70 \pm 0.56$ & $2.20 \pm 0.09$ \\
\hline 47 & 1998 Oct. 25 & 51112.259 & $1.34 \pm 0.04$ & & $2.11 \pm 0.09$ \\
\hline 48 & 1998 Dec. 02 & 51149.625 & $1.45 \pm 0.04$ & $7.68 \pm 0.65$ & $2.15 \pm 0.09$ \\
\hline 49 & 1999 Jul. 13 & 51372.516 & $1.19 \pm 0.04$ & & $1.37 \pm 0.06$ \\
\hline 50 & 1999 Aug. 19 & 51410.309 & $1.34 \pm 0.04$ & & $1.61 \pm 0.07$ \\
\hline 51 & 1999 Sep. 04 & 51425.904 & $1.43 \pm 0.04$ & & $1.65 \pm 0.07$ \\
\hline 52 & 1999 Sep. 05 & 51427.272 & & $7.40 \pm 0.62$ & \\
\hline 53 & 1999 Oct. 03 & 51454.674 & $1.62 \pm 0.05$ & & $1.89 \pm 0.08$ \\
\hline 54 & 1999 Oct. 04 & 51455.664 & $1.38 \pm 0.04$ & & $1.80 \pm 0.08$ \\
\hline 55 & 1999 Oct. 09 & 51461.531 & $1.55 \pm 0.05$ & & $1.65 \pm 0.07$ \\
\hline 56 & 2000 Jul. 19 & 51745.365 & $1.56 \pm 0.05$ & & $1.92 \pm 0.08$ \\
\hline 57 & 2000 Jul. 22 & 51748.232 & $1.60 \pm 0.05$ & & $1.56 \pm 0.07$ \\
\hline 58 & 2000 Jul. 30 & 51756.293 & $1.54 \pm 0.05$ & & $1.98 \pm 0.08$ \\
\hline
\end{tabular}


Table 2. continued.

\begin{tabular}{|c|c|c|c|c|c|}
\hline $\mathrm{N}$ & UT-date & $\begin{array}{c}\text { JD } \\
2400000+ \\
3\end{array}$ & $\begin{array}{c}F(5100) \pm \sigma \\
10^{-15} \mathrm{erg} \mathrm{cm}^{-2} \mathrm{~s}^{-1} \AA^{-1} \\
4\end{array}$ & $\begin{array}{c}F(\mathrm{H} \alpha) \pm \sigma \\
10^{-13} \mathrm{erg} \mathrm{cm}^{-2} \mathrm{~s}^{-1} \\
5\end{array}$ & $\begin{array}{c}F(\mathrm{H} \beta) \pm \sigma \\
10^{-13} \mathrm{erg} \mathrm{cm}^{-2} \mathrm{~s}^{-1} \\
6\end{array}$ \\
\hline 59 & 2000 Jul. 30 & 51756.317 & & $9.49 \pm 0.80$ & \\
\hline 60 & 2000 Oct. 05 & 51823.142 & $1.41 \pm 0.04$ & & $1.72 \pm 0.07$ \\
\hline 61 & 2000 Nov. 18 & 51867.136 & $1.56 \pm 0.05$ & & $1.82 \pm 0.08$ \\
\hline 62 & 2000 Nov. 19 & 51868.128 & $1.66 \pm 0.05$ & & $1.93 \pm 0.08$ \\
\hline 63 & 2001 Jan. 16 & 51925.631 & $1.53 \pm 0.05$ & & $1.93 \pm 0.08$ \\
\hline 64 & 2001 Jan. 16 & 51925.647 & & $7.78 \pm 0.65$ & \\
\hline 65 & 2001 Jan. 20 & 51929.623 & $1.47 \pm 0.04$ & & $1.81 \pm 0.08$ \\
\hline 66 & 2001 Jan. 20 & 51929.639 & & $9.59 \pm 0.81$ & \\
\hline 67 & 2001 Mar. 13 & 51981.950 & $1.40 \pm 0.04$ & & $1.88 \pm 0.08$ \\
\hline 68 & 2001 May 05 & 52034.660 & $1.39 \pm 0.04$ & $7.38 \pm 0.62$ & $1.56 \pm 0.07$ \\
\hline 69 & 2001 May 14 & 52043.900 & $1.35 \pm 0.04$ & $6.85 \pm 0.58$ & $1.56 \pm 0.07$ \\
\hline 70 & 2001 Jun. 14 & 52074.850 & $1.34 \pm 0.04$ & & $1.59 \pm 0.07$ \\
\hline 71 & 2001 Jun. 14.5 & 52075.350 & & $6.77 \pm 0.57$ & \\
\hline 72 & 2001 Jun. 15 & 52075.850 & $1.30 \pm 0.04$ & & $1.55 \pm 0.07$ \\
\hline 73 & 2001 Oct. 10 & 52192.600 & $1.08 \pm 0.03$ & $6.25 \pm 0.52$ & $1.22 \pm 0.05$ \\
\hline 74 & 2001 Nov. 23 & 52237.272 & $1.37 \pm 0.04$ & & $1.06 \pm 0.04$ \\
\hline 75 & 2002 Feb. 21 & 52327.226 & $1.36 \pm 0.04$ & & $1.09 \pm 0.05$ \\
\hline 76 & 2002 Feb. 21 & 52327.200 & & $6.63 \pm 0.56$ & \\
\hline 77 & 2002 Mar. 05 & 52339.019 & $1.20 \pm 0.04$ & $5.60 \pm 0.47$ & $0.94 \pm 0.04$ \\
\hline 78 & 2002 Apr. 03 & 52368.005 & $1.20 \pm 0.04$ & & $1.02 \pm 0.04$ \\
\hline 79 & 2002 Jun. 03 & 52428.968 & & $6.60 \pm 0.55$ & \\
\hline 80 & 2002 Jun. 04 & 52429.950 & & $6.47 \pm 0.54$ & \\
\hline 81 & 2002 Jun. 05 & 52430.938 & $1.42 \pm 0.04$ & & $1.21 \pm 0.05$ \\
\hline 82 & 2002 Jun. 24 & 52450.385 & $1.72 \pm 0.05$ & & $1.71 \pm 0.07$ \\
\hline 83 & 2002 Jun. 24 & 52450.410 & & $5.86 \pm 0.49$ & \\
\hline 84 & 2002 Jul. 09 & 52464.444 & $1.60 \pm 0.05$ & & $1.54 \pm 0.06$ \\
\hline 85 & 2002 Jul. 13 & 52469.408 & $1.85 \pm 0.06$ & & $1.72 \pm 0.07$ \\
\hline 86 & 2002 Jul. 13 & 52469.433 & & $5.93 \pm 0.50$ & \\
\hline 87 & 2002 Aug. 08 & 52495.272 & $1.71 \pm 0.05$ & & $1.42 \pm 0.06$ \\
\hline 88 & 2002 Aug. 16 & 52502.795 & $1.61 \pm 0.05$ & & $1.60 \pm 0.07$ \\
\hline 89 & 2002 Oct. 14 & 52562.318 & & $9.03 \pm 0.76$ & \\
\hline 90 & 2002 Oct. 14 & 52562.342 & $1.55 \pm 0.05$ & & $1.82 \pm 0.08$ \\
\hline 91 & 2002 Nov. 13 & 52591.659 & & $7.87 \pm 0.66$ & \\
\hline 92 & 2002 Nov. 14 & 52592.659 & & $6.32 \pm 0.53$ & \\
\hline 93 & 2002 Nov. 15 & 52593.671 & $1.36 \pm 0.04$ & & $1.60 \pm 0.07$ \\
\hline 94 & 2002 Dec. 11 & 52620.389 & $1.71 \pm 0.05$ & & $1.60 \pm 0.07$ \\
\hline 95 & 2003 May 08 & 52768.217 & $1.96 \pm 0.06$ & & $1.69 \pm 0.07$ \\
\hline 96 & 2003 May 10 & 52770.219 & & $6.40 \pm 0.54$ & \\
\hline 97 & 2003 May 23 & 52782.966 & $2.10 \pm 0.06$ & & $1.69 \pm 0.07$ \\
\hline 98 & 2003 May 24 & 52783.976 & $2.15 \pm 0.06$ & & $1.76 \pm 0.07$ \\
\hline 99 & 2003 May 25 & 52784.973 & & $6.68 \pm 0.56$ & \\
\hline 100 & 2003 May 26 & 52785.979 & $2.15 \pm 0.06$ & & $1.68 \pm 0.07$ \\
\hline 101 & 2003 Jun. 22 & 52812.963 & $2.28 \pm 0.07$ & & $1.78 \pm 0.07$ \\
\hline 102 & 2003 Sep. 18 & 52900.794 & & & $1.96 \pm 0.08$ \\
\hline 103 & 2003 Sep. 20 & 52902.803 & & $7.78 \pm 0.65$ & \\
\hline 104 & 2003 Oct. 21 & 52933.672 & $1.96 \pm 0.06$ & $7.11 \pm 0.60$ & $1.77 \pm 0.07$ \\
\hline 105 & 2003 Nov. 19 & 52962.637 & $2.83 \pm 0.08$ & & $1.99 \pm 0.08$ \\
\hline 106 & 2003 Nov. 20 & 52963.608 & $2.93 \pm 0.09$ & & $2.15 \pm 0.09$ \\
\hline 107 & 2004 Mar. 01 & 53066.358 & & $9.15 \pm 0.77$ & \\
\hline 108 & 2004 Mar. 01 & 53065.507 & $2.48 \pm 0.07$ & & $2.25 \pm 0.09$ \\
\hline 109 & 2004 May 20 & 53145.982 & $2.01 \pm 0.06$ & & $2.40 \pm 0.10$ \\
\hline 110 & 2004 Jun. 12 & 53168.955 & $1.95 \pm 0.06$ & & $2.32 \pm 0.10$ \\
\hline 111 & 2004 Jun. 13 & 53169.966 & & $8.71 \pm 0.73$ & \\
\hline 112 & 2004 Jun. 15 & 53171.944 & $1.95 \pm 0.06$ & & $2.04 \pm 0.09$ \\
\hline 113 & 2004 Aug. 19 & 53236.808 & $2.31 \pm 0.07$ & & $2.11 \pm 0.09$ \\
\hline 114 & 2004 Aug. 20 & 53237.821 & & $9.49 \pm 0.80$ & \\
\hline 115 & 2004 Aug. 21 & 53238.858 & $2.31 \pm 0.07$ & & $2.16 \pm 0.09$ \\
\hline 116 & 2004 Sep. 06 & 53254.771 & $2.13 \pm 0.06$ & & $2.16 \pm 0.09$ \\
\hline 117 & 2004 Dec. 18 & 53358.140 & $3.13 \pm 0.09$ & & $2.78 \pm 0.12$ \\
\hline
\end{tabular}


Table 2. continued.

\begin{tabular}{|c|c|c|c|c|c|}
\hline $\mathrm{N}$ & UT-date & $\begin{array}{c}\text { JD } \\
2400000+ \\
3\end{array}$ & $\begin{array}{c}F(5100) \pm \sigma \\
10^{-15} \mathrm{erg} \mathrm{cm}^{-2} \mathrm{~s}^{-1} \AA^{-1} \\
4\end{array}$ & $\begin{array}{c}F(\mathrm{H} \alpha) \pm \sigma \\
10^{-13} \underset{5}{\mathrm{erg} \mathrm{\textrm {cm } ^ { - 2 }} \mathrm{s}^{-1}}\end{array}$ & $\begin{array}{c}F(\mathrm{H} \beta) \pm \sigma \\
10^{-13} \mathrm{erg} \mathrm{cm}^{-2} \mathrm{~s}^{-1} \\
6\end{array}$ \\
\hline 118 & 2004 Dec. 19 & 53359.130 & $2.92 \pm 0.09$ & $10.69 \pm 0.90$ & $2.67 \pm 0.11$ \\
\hline 119 & 2004 Dec. 20 & 53360.150 & $2.87 \pm 0.09$ & $11.23 \pm 0.94$ & $2.76 \pm 0.12$ \\
\hline 120 & 2005 Apr. 15 & 53475.997 & $3.19 \pm 0.1$ & & $2.71 \pm 0.11$ \\
\hline 121 & 2005 Apr. 16 & 53476.993 & & $9.69 \pm 0.81$ & \\
\hline 122 & 2005 Apr. 18 & 53478.992 & $3.28 \pm 0.1$ & $10.11 \pm 0.85$ & $2.99 \pm 0.13$ \\
\hline 123 & 2005 May 13 & 53503.972 & $3.66 \pm 0.11$ & & $2.92 \pm 0.12$ \\
\hline 124 & 2005 Jun. 10 & 53531.925 & $4.25 \pm 0.13$ & & $2.91 \pm 0.12$ \\
\hline 125 & 2005 Aug. 30 & 53612.758 & $4.57 \pm 0.14$ & & $3.50 \pm 0.15$ \\
\hline 126 & 2005 Aug. 31 & 53613.773 & $4.55 \pm 0.14$ & & $3.34 \pm 0.14$ \\
\hline 127 & 2005 Sep. 01 & 53614.750 & $4.62 \pm 0.14$ & & $3.54 \pm 0.15$ \\
\hline 128 & 2005 Dec. 06 & 53711.420 & $4.63 \pm 0.14$ & $19.14 \pm 1.61$ & $4.09 \pm 0.17$ \\
\hline 129 & 2006 Jun. 04 & 53891.490 & $3.71 \pm 0.11$ & & $3.70 \pm 0.16$ \\
\hline 130 & 2006 Sep. 01 & 53979.790 & $3.24 \pm 0.1$ & & $3.49 \pm 0.15$ \\
\hline 131 & 2006 Sep. 16 & 53994.780 & $3.12 \pm 0.09$ & & $3.15 \pm 0.13$ \\
\hline 132 & 2006 Sep. 17 & 53995.750 & $3.17 \pm 0.1$ & & $3.40 \pm 0.14$ \\
\hline 133 & 2006 Sep. 18 & 53996.700 & $3.20 \pm 0.1$ & & $3.40 \pm 0.14$ \\
\hline 134 & 2006 Sep. 19 & 53997.740 & $3.20 \pm 0.1$ & & $3.44 \pm 0.14$ \\
\hline 135 & 2006 Sep. 20 & 53998.750 & $3.18 \pm 0.1$ & & $3.27 \pm 0.14$ \\
\hline 136 & 2006 Oct. 28 & 54036.630 & $2.87 \pm 0.09$ & $17.41 \pm 1.46$ & $3.18 \pm 0.13$ \\
\hline 137 & 2006 Oct. 29 & 54037.640 & $2.82 \pm 0.08$ & & $3.40 \pm 0.14$ \\
\hline 138 & 2006 Nov. 01 & 54040.650 & $2.84 \pm 0.09$ & & $3.30 \pm 0.14$ \\
\hline 139 & 2006 Dec. 18 & 54088.210 & $2.99 \pm 0.09$ & & $3.31 \pm 0.14$ \\
\hline 140 & 2007 Jan. 12 & 54112.640 & $3.46 \pm 0.1$ & $12.16 \pm 1.02$ & $3.18 \pm 0.13$ \\
\hline 141 & 2007 Jan. 13 & 54113.650 & $3.32 \pm 0.1$ & $14.72 \pm 1.24$ & $3.14 \pm 0.13$ \\
\hline 142 & 2007 Feb. 12 & 54143.610 & $3.92 \pm 0.12$ & $15.86 \pm 1.33$ & $3.35 \pm 0.14$ \\
\hline 143 & 2007 May 22 & 54242.890 & $4.89 \pm 0.15$ & & $3.72 \pm 0.16$ \\
\hline 144 & 2007 May 23 & 54243.880 & $5.07 \pm 0.15$ & & $3.97 \pm 0.17$ \\
\hline 145 & 2007 Jun. 20 & 54271.870 & $4.85 \pm 0.15$ & & $3.83 \pm 0.16$ \\
\hline 146 & 2007 Jun. 21 & 54272.960 & $4.72 \pm 0.14$ & & $4.20 \pm 0.18$ \\
\hline 147 & 2007 Aug. 09 & 54321.820 & $3.73 \pm 0.11$ & & $3.96 \pm 0.17$ \\
\hline 148 & 2007 Aug. 10 & 54322.810 & & $12.46 \pm 1.05$ & \\
\hline 149 & 2007 Aug. 11 & 54323.800 & $3.78 \pm 0.11$ & & $4.06 \pm 0.17$ \\
\hline 150 & 2007 Aug. 12 & 54324.810 & $3.81 \pm 0.11$ & & $3.94 \pm 0.17$ \\
\hline 151 & 2007 Sep. 03 & 54346.730 & $4.11 \pm 0.12$ & $15.30 \pm 1.28$ & $4.28 \pm 0.18$ \\
\hline 152 & 2007 Sep. 04 & 54347.720 & $4.09 \pm 0.12$ & & $4.30 \pm 0.18$ \\
\hline 153 & 2007 Sep. 05 & 54348.720 & $4.10 \pm 0.12$ & & $4.08 \pm 0.17$ \\
\hline 154 & 2007 Oct. 16 & 54389.690 & $3.91 \pm 0.12$ & $13.96 \pm 1.17$ & $4.34 \pm 0.18$ \\
\hline 155 & 2007 Oct. 18 & 54391.630 & $4.05 \pm 0.12$ & & $4.42 \pm 0.19$ \\
\hline 156 & 2007 Nov. 02 & 54406.650 & $3.80 \pm 0.11$ & & $4.10 \pm 0.17$ \\
\hline 157 & 2007 Nov. 04 & 54408.610 & $3.78 \pm 0.11$ & $12.81 \pm 1.08$ & $4.28 \pm 0.18$ \\
\hline 158 & 2007 Nov. 08 & 54412.690 & $3.67 \pm 0.11$ & & $4.12 \pm 0.17$ \\
\hline
\end{tabular}

Notes. Column (1): number. Column (2): UT-date. Column (3): Julian date. Column (4): $F$ (cont), the continuum flux at $5100 \AA$ (for $Z=0$ ) (in units of $10^{-15} \mathrm{erg} \mathrm{s}^{-1} \mathrm{~cm}^{-2} \AA^{-1}$ ), and $\varepsilon_{\text {cont }}$, the estimated continuum flux error. Column $(5): F(\mathrm{H} \alpha)$, the $\mathrm{H} \alpha$ total flux (in units of $\left.10^{-13} \mathrm{erg} \mathrm{s}^{-1} \mathrm{~cm}^{-2}\right)$ in regions (6740-7160) $\AA$, and $\varepsilon_{\mathrm{H} \alpha}$, the $\mathrm{H} \alpha$ flux error. Column (6): $F(\mathrm{H} \beta)$, the $\mathrm{H} \beta$ total flux (in units of $10^{-13} \mathrm{erg} \mathrm{s}^{-1} \mathrm{~cm}^{-2}$ ) in regions (4909-5353) $\AA$, and $\varepsilon_{\mathrm{H} \beta}$, the $\mathrm{H} \beta$ flux error. 